\title{
Investir quand cela compte: Passer en revue les preuves et établir un plan de recherche et d'action pour les très jeunes adolescents
}

\author{
Katharine McCarthy \\ Population Council \\ Martha Brady \\ Population Council \\ Kelly Hallman \\ Population Council
}

Follow this and additional works at: https://knowledgecommons.popcouncil.org/departments_sbsr-pgy

Part of the Demography, Population, and Ecology Commons, Family, Life Course, and Society Commons, and the International Public Health Commons How does access to this work benefit you? Let us know!

\section{Recommended Citation}

McCarthy, Katharine, Martha Brady, and Kelly Hallman. 2016. "Investir quand cela compte: Passer en revue les preuves et établir un plan de recherche et d'action pour les très jeunes adolescents." New York: Population Council. 


\section{INVESTIR \\ QUAND CELA COMPTE}

Passer en revue les preuves et établir un plan de recherche et d'action pour les très jeunes adolescents
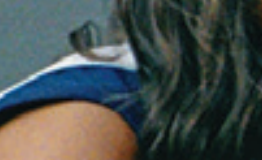



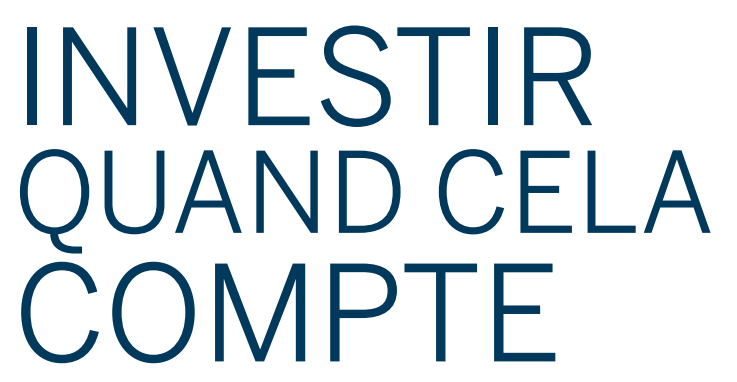

Passer en revue les preuves et établir un plan de recherche et d'action pour les très jeunes adolescents

KATHARINE MCCARTHY

MARTHA BRADY

KELLY HALLMAN 


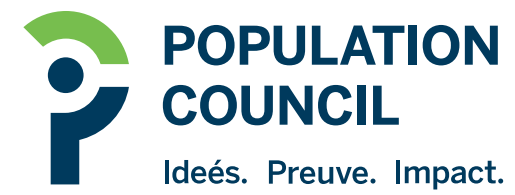

Le Population Council s'attelle aux questions cruciales de la santé et du développement - qu'il s'agisse d'endiguer la progression du VIH, d'améliorer la santé reproductive et d'offrir aux jeunes la promesse d'une vie satisfaisante et productive. Par ses travaux de recherche en biomédecine, en sciences sociales et en santé publique dans 50 pays, il s'efforce d'apporter avec ses partenaires des solutions qui conduisent à des politiques, des programmes et des technologies plus efficaces, pour améliorer la vie partout dans le monde. Fondé en 1952, le Population Council est une organisation non gouvernementale à but non lucratif. II siège à New York sous la conduite d'un conseil d'administration international.

Population Council

One Dag Hammarskjold Plaza

New York, NY 10017

\section{popcouncil.org}

Ces travaux ont été rendus possibles grâce au généreux soutien de la fondation John D. et Catherine T. MacArthur.

Citation suggérée: McCarthy, Katharine, Martha Brady, et Kelly Hallman. 2016. "Investir quand cela compte: Passer en revue les preuves et établir un plan de recherche et d'action pour les très jeunes adolescents." New York: Population Council.

Crédits photo-couverture: ( ) Gabriel Amadeus Cooney; page 1: Sam Loewenberg; page 9: Nadia Zibani; page 17: Chris Stowers/Panos; page 33: Petrut Calinescu/Panos; page 53: Josh Estey/CARE; page 63: Andrea Lynch/IWHC

ISBN: 978-0-87834-135-3

Imprimé aux États-Unis d'Amérique.

(c) 2016 The Population Council, Inc. 


\section{Contenu}

Figures, encadrés et tableaux

Abréviations

Avant-propos

Remerciements

Préface

\section{Introduction}

1.1 Investir dans les très jeunes adolescentes et adolescents: pourquoi c'est important

1.2 Changements sociaux, biologiques et développementaux au début de l'adolescence

2 La santé et le bien-être des TJA : vue d'ensemble et état actuel

2.1 La maladie et l'incapacité: comment s'en sortent les jeunes adolescentes et adolescents?

2.2 Comprendre les comportements des TJA en matière de santé pour endiguer la propagation des maladies non transmissibles

2.3 Les résultats de santé pour les TJA: le monde divergent des filles et des garçons

3 Influences sociales et contextuelles sur la santé : preuves et observations

3.1 Les domaines critiques dans la vie des jeunes adolescentes et adolescents

3.2 Différences liées au genre dans la transition de l'école primaire à l'école secondaire

3.3 Survie et présence des parents

3.4 Le rôle des normes de genre au début de l'adolescence

3.5 La violence à l'égard des TJA: les garçons et les filles sont touchés, mais la prévalence varie en fonction du genre iv

iv

V

vi

vii

1

2

3

9

10

11

18

21

22

23

page

3.6 La scolarité et la violence

page

3.7 Les programmes répondant aux TJA dans des circonstances graves et particulières

4 Progresser : améliorer les programmes pour les TJA

4.1 Identifier les principaux domaines pour les programmes destinés aux TJA

4.2 Programmes pour lesquels nous disposons de preuves solides

4.3 Programmes prometteurs, mais pour lesquels il est nécessaire d'obtenir davantage de preuves

5 Les recherches auprès des TJA: recueillir des informations pour guider les programmes et les politiques

5.1 Études incluant les données disponibles sur les TJA

5.2 Considérations éthiques dans les recherches effectuées directement auprès des TJA

5.3 Méthodes et outils de recherche

6 Conclusion

6.1 Les interventions programmatiques à tester

6.2 Regarder vers l'avenir

66

Postface: Le moment est venu

Références

69 


\section{Figures, encadrés et tableaux}

FIGURES

1 L'adolescence influence la santé tout au long de la vie

2 Pourcentage de la population mondiale âgée de 10 à 14 ans, par pays, 2012

3 Les étapes de l'adolescence (classification de I'OPS)

4 Le début de l'adolescence: Iorsque la vie des filles prend une mauvaise direction

5 Pourcentage d'adolescents scolarisés, âgés entre 10 et 14 ans et 15 et 19 ans, quatre pays sélectionnés, 2004-2009

6 Le cadre écologique au cours de la vie

7 Pourcentage de femmes âgées de 20 à 24 ans qui étaient mariées avant l'âge de 15 ans, Éthiopie, 2005

8 Pourcentage de filles âgées de 10 à 14 ans non scolarisées et ne vivant avec aucun de leurs parents, Guatemala, 2002/2006

\section{ENCADRÉS}

1 Programmes d'enquête majeurs présentant des données de répondants de plus de 14 ans qui comprennent desinformations sur les TJA

2 Programmes d'enquêtes majeurs axés principalement sur le groupe d'âge des TJA page

\section{Abréviations}

ACASI Auto-entretien audio assisté par ordinateur (Audio Computer-Assisted Self-Interview)

AVCI Année de vie corrigée de l'incapacité

CDC Centres pour le contrôle et la prévention des maladies (Centers for Disease Control and Prevention)

ECS Éducation complète à la sexualité

EDS Enquête démographique et de santé

GSHS Enquête mondiale sur la santé des élèves en milieu scolaire (Global School-Based Health Survey)

ISPCAN Société internationale pour la prévention des mauvais traitements et négligences envers les enfants (International Society for Prevention of Child Abuse and Neglect)

IST Infection sexuellement transmissible

LSE Éducation basée sur l'acquisition de compétences nécessaires dans la vie courante (Life Skills Education)
3 Les recherches devraient-elles inclure les TJA?

4 Des entretiens en face à face ou assistés par ordinateur?

\section{TABLEAUX}

1 Les étapes du développement des très jeunes adolescents, par âge et par genre

2 Les résultats liés à la SSR avant l'âge de 15 ans tels que rapportés par des adolescentes de 15 à 19 ans, par pays

3 Caractéristiques des très jeunes adolescents basées sur les données d'EDS récentes, incluant la résidence, la présence parentale, et la fréquentation et les résultats scolaires, par région

4 Percent of adolescents reporting any unwanted sexual experience in previous year, according to type of survey

5 Les exemples d'éléments d'un «contrôle de santé» pour les TJA doivent être adaptés aux filles et aux garçons

6 Les facteurs de risque et de protection associés à la santé mentale des adolescents
MICS Enquêtes en grappes à indicateur multiple (Multiple Indicator Cluster Survey)

MNT Maladie non transmissible

PHR Pays à haut revenu

PRFM Pays à revenu faible ou moyen

SIDA Syndrome d'immunodéficience acquise

SSR Santé sexuelle et reproductive

TIC Technologies de l'information et de la communication

TJA Très jeune adolescent

UNFPA Fonds des Nations unies pour la population UNICEF Fonds des Nations unies pour l'enfance

VACS Enquête sur la violence contre les enfants

VIH Virus de l'immunodéficience humaine 


\section{Avant-propos}

Les années entre 10 et 14 ans représentent une période cruciale dans la transition de l'enfance à l'adolescence. Ces années de développement rapide sont marquées par l'acquisition de connaissances importantes dans le domaine social et de la santé, l'adoption des comportements, le façonnement des croyances et des attitudes, et c'est pendant ces années que les bases de l'âge adulte sont jetées.

Au cours de ces 20 dernières années, depuis que le Population Council a commencé à rassembler un ensemble de preuves sur la vie des adolescents, il est devenu évident que les «adolescents » ne sont pas un groupe homogène. Au contraire, il est important d'observer les divers éléments qui différencient les filles et les garçons en fonction de leur situation matrimoniale, de l'endroit où ils vivent, des expériences qu'ils connaissent et de nombreuses autres caractéristiques. L'âge et le genre sont des caractéristiques particulièrement distinctives, comme ce rapport, qui se concentre sur le tout début de la deuxième décennie de la vie, l'établit clairement. De nombreux éléments distinguent les adolescents de 10 à 14 ans des adolescents plus âgés, et l'expérience des garçons et des filles est marquée par des différences profondes; le fait de prendre ces différences en compte au moment de penser les interventions est essentiel pour améliorer leur impact.

Comme les très jeunes adolescents sont généralement en bonne santé, peu de programmes leur ont été consacrés. Mais au fil des nouvelles connaissances sur le développement cognitif et social, l'importance de ces années est devenue de plus en plus évidente. Les interventions des programmes visant à influencer les transitions essentielles qui se produisent au début de l'adolescence peuvent entraîner un effet positif et durable, mais il est nécessaire d'accorder une attention plus systématique à la conception et à l'évaluation des programmes, en prenant en compte la diversité de ce groupe.

Le Population Council effectue des recherches, et conçoit et évalue des programmes destinés aux adolescents depuis des décennies. Depuis la publication du premier rapport Investing When It Counts il y a 10 ans, nos travaux sur et avec les très jeunes adolescents se sont développés, tout comme les travaux de nombreuses autres organisations. Cela a entraîné une meilleure compréhension des besoins et opportunités de ce groupe d'âge, à mesure que l'obtention de données plus nombreuses et de meilleure qualité a clarifié leur situation. Ce rapport présente les données et les éléments de preuve les plus récents sur la vie des adolescents pendant cette période clé de transition vers l'âge adulte. Nous nous engageons à poursuivre nos efforts en vue de comprendre la vie des jeunes adolescents, pour que les investissements qui leur sont consacrés soient aussi efficaces que possible.

ANN K. BLANC, DR VICE-PRÉSIDENTE ET DIRECTRICE PROGRAMME PAUVRETÉ, GENRE ET JEUNESSE POPULATION COUNCIL 


\section{Remerciements}

Ce rapport a bénéficié des idées et contributions de nombreuses personnes. Nous exprimons notre reconnaissance à Michelle Chau, qui a élaboré les codes et effectué les analyses initiales des données d'EDS. Nous tenons également à remercier sincèrement les nombreuses personnes qui ont participé à l'élaboration des différentes sections, notamment: Vira David pour son travail sur la cartographie spatiale, Stephanie Psaki pour avoir révisé et proposé des idées sur la section liée à l'éducation, Rebecka Lundgren pour avoir révisé et contribué à la section sur l'élaboration des programmes, et Angel del Valle qui a fourni des conseils sur les méthodes de recherche participative. Merci à Joyce Altman et Mike Vosika qui ont participé à la révision et à l'élaboration de ce rapport. Enfin, nous remercions les personnes qui ont revu avec beaucoup de soin et d'attention ce rapport, et qui ont apporté des contributions importantes à sa forme et à sa texture globales. Merci notamment à: Ann Blanc, Judith Bruce, Sarah Engebretsen, Sara Peracca et Miriam Temin. 


\section{Préface}

En2006, le Population Council a publié, en partenariat avec l'UNFPA, le rapport Investing When It Counts: Generating the Evidence Base for Policies and Programmes for Very Young Adolescents, qui était le premier effort majeur de synthèse des informations sur les adolescentes et adolescents de 10 à 14 ans, et utilisait des ensembles de données existants, des recherches et l'expérience des programmes (Chong, Hallman et Brady 2006). Depuis, nous avons pu constater un intérêt grandissant pour la population des très jeunes adolescents (TJA), ainsi qu'une expansion continue des programmes visant à répondre à leurs besoins sociaux, de santé et de développement. Le rapport actuel s'appuie sur la dynamique engendrée par les recherches et les efforts récents déployés dans le cadre des programmes, et le volume plus important de données générées au cours de la dernière décennie.

Ce rapport se veut utile à toute personne chargée de planifier, gérer, mettre en œuvre, suivre, évaluer ou financer des recherches ou des programmes impliquant les jeunes adolescents et adolescentes. Nos travaux sont axés sur les résultats et les facteurs d'influence en matière de santé et de bien-être. Bien que le rapport présente des données mondiales concernant les TJA, nous nous concentrons principalement sur les résultats des pays à revenu faible ou moyen (PRFM), et dans la mesure du possible, ventilés par sexe.

Le rapport est organisé en cinq sections principales. Dans I'«Introduction», nous expliquons la raison pour laquelle il est important d'intervenir auprès des TJA, étant donné les transitions nombreuses, rapides et potentiellement irrémédiables qui se produisent pendant cette période de la vie, ainsi que l'apparition des différences entre les sexes à partir de ce stade. Nous résumons ce que nous savons sur les jeunes adolescentes et adolescents, et examinons les manières dont leurs trajectoires de vie commencent à prendre forme et à diverger. Nous nous concentrons sur les principaux résultats en matière de santé et sur les facteurs sociaux et contextuels qui en sont à l'origine. Dans "La santé et le bien-être des TJA», nous présentons des informations à l'échelle mondiale sur la maladie et l'incapacité chez les jeunes adolescents, et discutons des questions de santé particulièrement pertinentes pendant l'adolescence.

Dans «Les influences sociales et contextuelles sur la santé », nous abordons la manière dont les principales influences dans l'environnement immédiat des adolescents déterminent leur développement global, leur bien-être et leur santé. Cette section s'appuie sur des sources très diverses, notamment des examens récents sur l'état de santé des adolescents, publiés dans The Lancet en 2012, le Rapport sur l'état de la population mondiale de I'UNFPA de 2014, et la publication de I'UNICEF «Progrès pour les enfants » de 2012, entre autres. Nous présentons également une analyse actualisée des données des enquêtes démographiques et de santé(EDS), et mettons en évidence les tendances de cette dernière décennie concernant les facteurs d'influence et résultats clés pertinents pour la période de 10 à 14 ans. De plus, nous nous basons sur les idées et les informations tirées des publications et ressources du Population Council, indiquées dans l'encadré intitulé « Ressources du Population Council sur l'adolescence».

Dans «Progresser: améliorer les programmes pour les TJA», nous examinons les preuves liées à l'amélioration des principaux domaines de la santé et du bien-être des adolescents, et décrivons les programmes ou les interventions visant à agir sur ces domaines.

Dans «Recherches auprès des TJA: recueillir des informations pour guider les programmes et 
RESSOURCES DU POPULATION COUNCIL SUR L'ADOLESCENCE

\section{OUTILS POUR LES PROGRAMMES ET LES RECHERCHES}

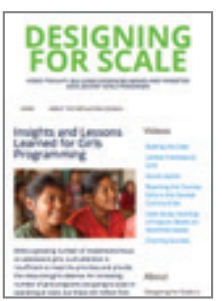

Designing for Scale:

Video Toolkit on Building

Evidence-Based and

Targeted Adolescent Girls

Programs

designingforscale.popcouncil.org

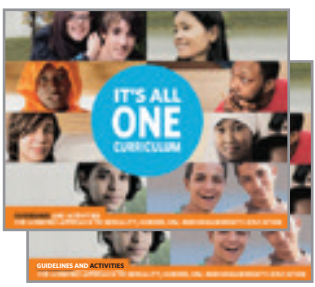

It's All One Curriculum:

Guidelines and Activities

for a Unified Approach

to Sexuality, Gender,

HIV, and Human Rights

Education

popcouncil.org/itsallone

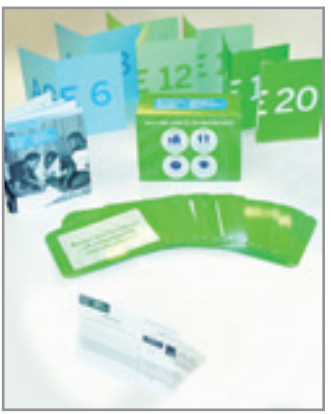

Building

Assets Toolkit:

Developing Positive

Benchmarks for

Adolescent Girls

popcouncil.org/

assettoolkit

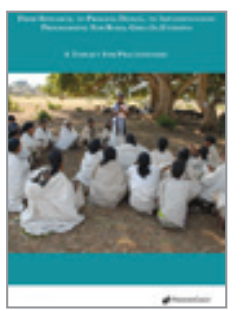

From Research, to Program Design, to Implementation:

Programming for Rural Girls in Ethiopia

popcouncil.org/

ethiopiagirlsprogram

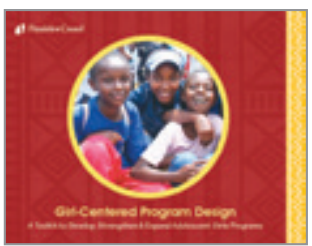

Girl-Centered Program

Design: A Toolkit to

Develop, Strengthen,

and Expand Adolescent

Girls Programs

popcouncil.org/

girlcenteredprogram

\section{PUBLICATIONS DE RECHERCHE}

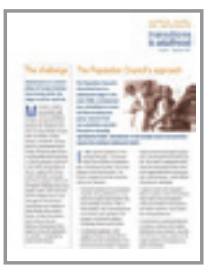

Brief Series: Promoting Healthy, Safe, and

Productive Transitions to Adulthood

popcouncil.org/

transitionbriefs

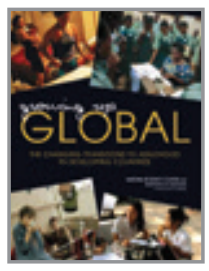

Growing Up Global:

The Changing Transitions

to Adulthood in Developing

Countries

popcouncil.org/

growingupglobal

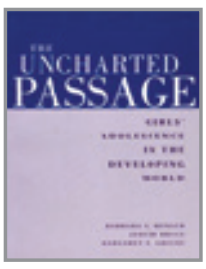

The Uncharted Passage:

Girls' Adolescence in the

Developing World

popcouncil.org/

unchartedpassage

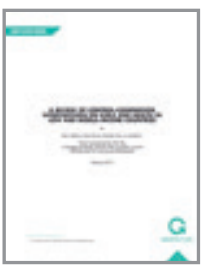

A Review of Control-

Comparison Interventions on Girls and Health in Low-

\& Middle-Income Countries

popcouncil.org/

interventiongirls

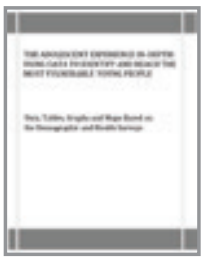

The Adolescent Experience In-depth-Using Data to Identify and Reach the Most Vulnerable Young People (Data Guides)

popcouncil.org/ adolgirlsdataguides

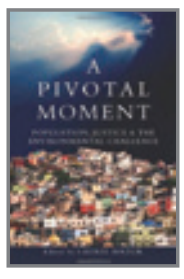

Chapitre d'un livre: "The new population challenge" (Judith Bruce et John Bongaarts)

dans A Pivotal Moment: Population, Justice, and the Environmental Challenge 
les politiques », nous nous concentrons sur les approches en matière de recherche, les sources de données existantes et les outils de données pouvant être utilisés pour élargir la base de connaissances sur les jeunes adolescents. Nous abordons également les points sensibles et des défis éthiques liés à l'organisation de recherches avec des filles et des garçons de ce groupe d'âge. Nous fournissons une liste élargie d'approches et de méthodes de collecte des données incluant de nouvelles techniques comme la cartographie, les exercices liés à la couverture des programmes et d'autres outils pratiques. Cette section établit également la liste des principales sources d'outils de données et de ressources liés aux recherches avec les TJA. La «Conclusion» fournit un résumé des recommandations clés pour améliorer les enseignements et les recherches dans le cadre des programmes.

Dans l'ensemble du rapport, nous présentons un large éventail d'approches, mais nous reconnaissons que les méthodologies de recherche doivent être adaptées spécifiquement à la culture, à l'âge et au genre. Les chercheurs doivent également travailler en étroite collaboration avec les membres de la communauté pour planifier et mettre en œuvre les recherches, diffuser les résultats et développer des solutions. Dans l'idéal toute étude portant sur ce groupe d'âge ventilera les données par genre et par étape de développement des adolescents au sein de la tranche d'âge de cinq ans couverte par les TJA (Section 1.2).

Nous adoptons une optique basée sur le genre en décrivant les différences en matière d'influences sociales et d'effets sur la santé pour les garçons et les filles, dans la mesure du possible. Nous sommes convaincus que cette perspective est importante, car cette phase du cycle de vie est un moment où la vie des filles et des garçons commence à diverger considérablement en termes de scolarité, de mobilité spatiale, de responsabilités familiales et, dans certains pays, de situation matrimoniale. C'est également le moment où les disparités entre les sexes apparaissent concernant l'accès aux ressources et aux opportunités économiques et institutionnelles. De plus, dans la plupart des contextes, le rang des filles est inférieur à celui des garçons dans quasiment tous les

\section{Les objectifs de ce rapport}

- Attirer l'attention sur les besoins particuliers, les défis et les risques émergents des jeunes adolescentes et adolescents

- Illustrer comment les événements de la très jeune adolescence (TJA) influencent les trajectoires de vie

- Éclairer les dimensions sociales et liées à la santé dans la vie des TJA, grâce à des statistiques EDS actualisées pour 71 pays et des données au niveau infranational sur des sujets précis

- Résumer les efforts visant à améliorer les résultats, ventilés par genre et par âge

- Identifier les lacunes en matière de preuves et les domaines d'investissement

- Décrire les sources de données et les approches utilisées pour recueillir des informations sur les TJA; fournir des outils et des ressources pratiques

- Proposer des recommandations pour les recherches et l'élaboration de programmes futurs

domaines; les filles ont moins de pouvoir et font face à des risques disproportionnés, ancrés dans l'inégalité entre les sexes. C'est pendant cette étape de la vie que ces inégalités commencent en grande partie à prendre forme et à se consolider. L'attention accordée au genre à cette étape de la vie peut donc permettre de cibler de manière plus précise les efforts de recherche et les interventions des programmes. Bien que nous ayons cherché à inclure aussi bien les garçons que les filles dans notre examen, nous mettons davantage l'accent sur les filles car les effets négatifs, en particulier liés à la santé sexuelle et reproductive, sont souvent plus profonds pour les filles, et peuvent être irrémédiables et changer leur vie. 



\section{Introduction}

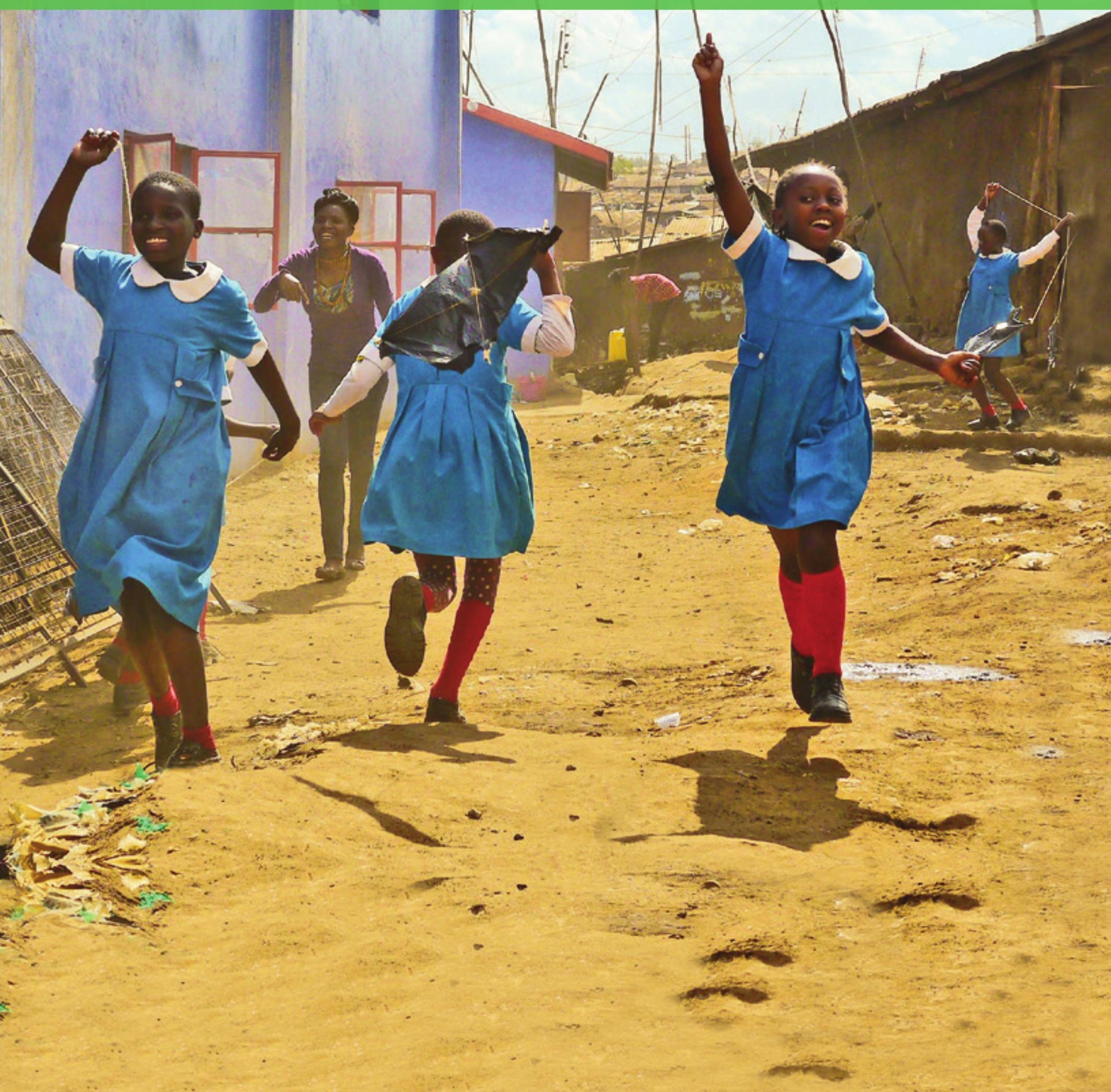




\subsection{Investir dans les très jeunes adolescentes et adolescents: pourquoi c'est important}

Se concentrer sur les jeunes adolescents est un investissement judicieux. C'est en effet la période où sont adoptés des comportements de santé qui dureront toute la vie, où apparaissent des voies présentant des opportunités ou des risques, et où le parcours de vie commence à prendre forme. Les changements développementaux et sociaux liés au début de l'adolescence peuvent avoir une influence durable sur les trajectoires qui seront empruntées plus tard par les jeunes (Figure1). Bien que la période de l'adolescence s'étende sur 10 ans, le début de l'adolescence ou la jeune adolescence (la phase de la vie entre l'âge de 10 et 14 ans) est un moment de transitions particulièrement dynamiques et rapides. Le fait de réunir des preuves sur le moment, la nature et les conséquences des transitions clés par lesquelles passent les jeunes adolescents est essentiel pour développer des politiques et des programmes adaptés à cette population. Le choix de moments stratégiques pour mettre en œuvre les interventions permet d'obtenir des résultats positifs avant que l'architecture de la vie des adolescents ne soit déterminée.

En2015, on comptait environ 1,2 milliards d'adolescents âgés de 10 à 19 ans dans le monde, soit environ $16 \%$ de la population mondiale (UNDESA 2013). Près de la moitié des adolescents dans le monde (0,6 milliard) sont au début de l'adolescence; ils sont également désignés sous le nom de «très jeunes adolescents» (TJA) (UNDESA 2013). La grande majorité des adolescents dans le monde, environ $90 \%$, résident dans les pays à revenu faible ou moyen (PRFM), où les TJA représentent un pourcentage encore plus important de la population et où les obstacles à une bonne santé et au bien-être sont souvent complexes. Le segment des TJA dans la population totale est le plus élevé dans les pays d'Afrique subsaharienne où, contrairement à d'autres régions du monde, la population jeune devrait continuer à croître au cours des quelques prochaines décennies (Figure 2) (ONU 2015). Cela signifie que les pays qui ont déjà des difficultés à répondre aux besoins des TJA en matière de santé et d'éducation auront besoin d'y consacrer davantage de ressources au cours des prochaines années pour permettre aux jeunes d'atteindre une bonne santé et le bien-être.

\section{FIGURE 1 L'adolescence influence la santé tout au long de la vie}

\section{Politiques et interventions sociales, éducatives et économiques}

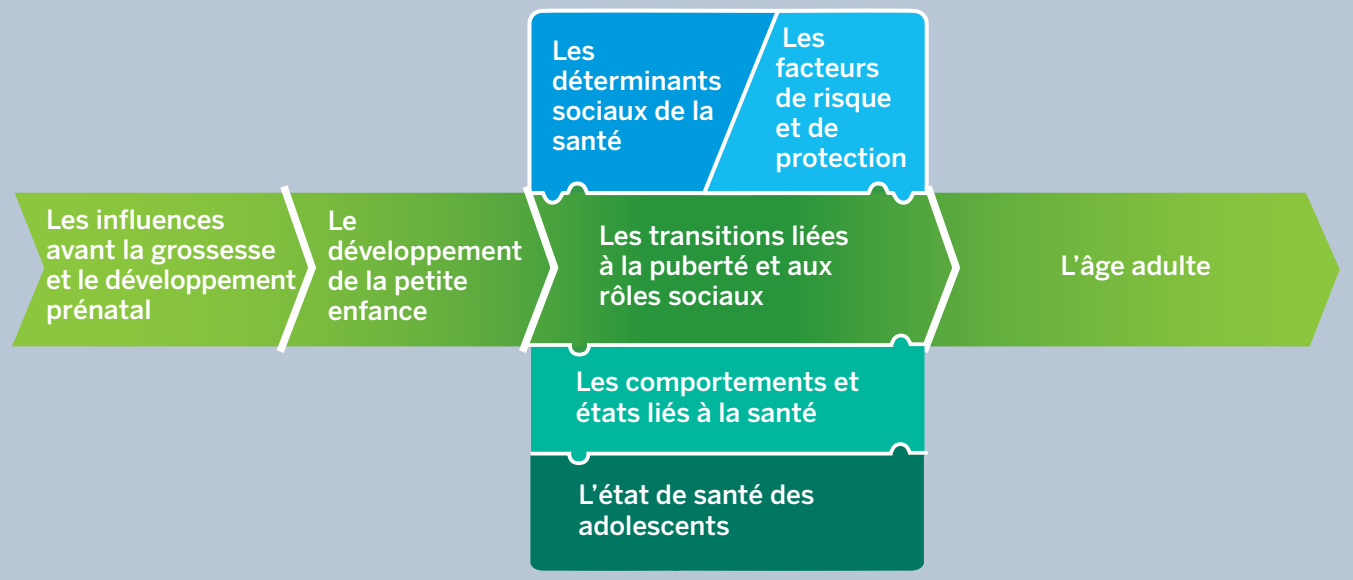

Politiques de santé

Prestation de soins préventifs et de services de santé 
Les TJA ont reçu beaucoup moins d'attention de la part des programmes et des politiques que les enfants plus jeunes et les adolescents plus âgés. Ce manque d'attention témoigne du fait que le début de l'adolescence est généralement une période marquée par une relative bonne santé. Cependant, comme cela est décrit dans cette section, le début de l'adolescence est un moment crucial pendant lequel l'exposition aux influences négatives et la perte de droits peuvent entraîner les vies sur une mauvaise voie, même si parfois les effets de ces expositions nocives sur la santé ne se manifestent que plus tard au cours de la vie. On reconnait de plus en plus le besoin essentiel de protéger la santé et les droits des filles et des garçons pendant cette période, afin de promouvoir une transition saine vers l'âge adulte (Sawyer et al. 2012; UNICEF 2012c). Dans les sections qui suivent, nous décrivons les transitions dynamiques qui définissent le début de l'adolescence et les opportunités et les risques présentés par ces changements pour influencer la santé actuelle et future.

\section{La grande majorité des adolescents dans le monde, environ $90 \%$, résident dans les pays à revenu faible ou moyen.}

\subsection{Changements sociaux, biologiques et développe- mentaux au début de l'adolescence}

Entre l'âge de 10 et 14 ans, les enfants subissent des changements physiques, émotionnels, sociaux et cognitifs immenses, et sont socialisés aux normes de genre prédominantes. À travers le monde, les jeunes font l'expérience de ces processus de manière unique et à différents moments, déterminés à la fois par les facteurs individuels et contextuels.

\section{FIGURE 2 Pourcentage de la population mondiale âgée de 10 à 14 ans, par pays, 2012}

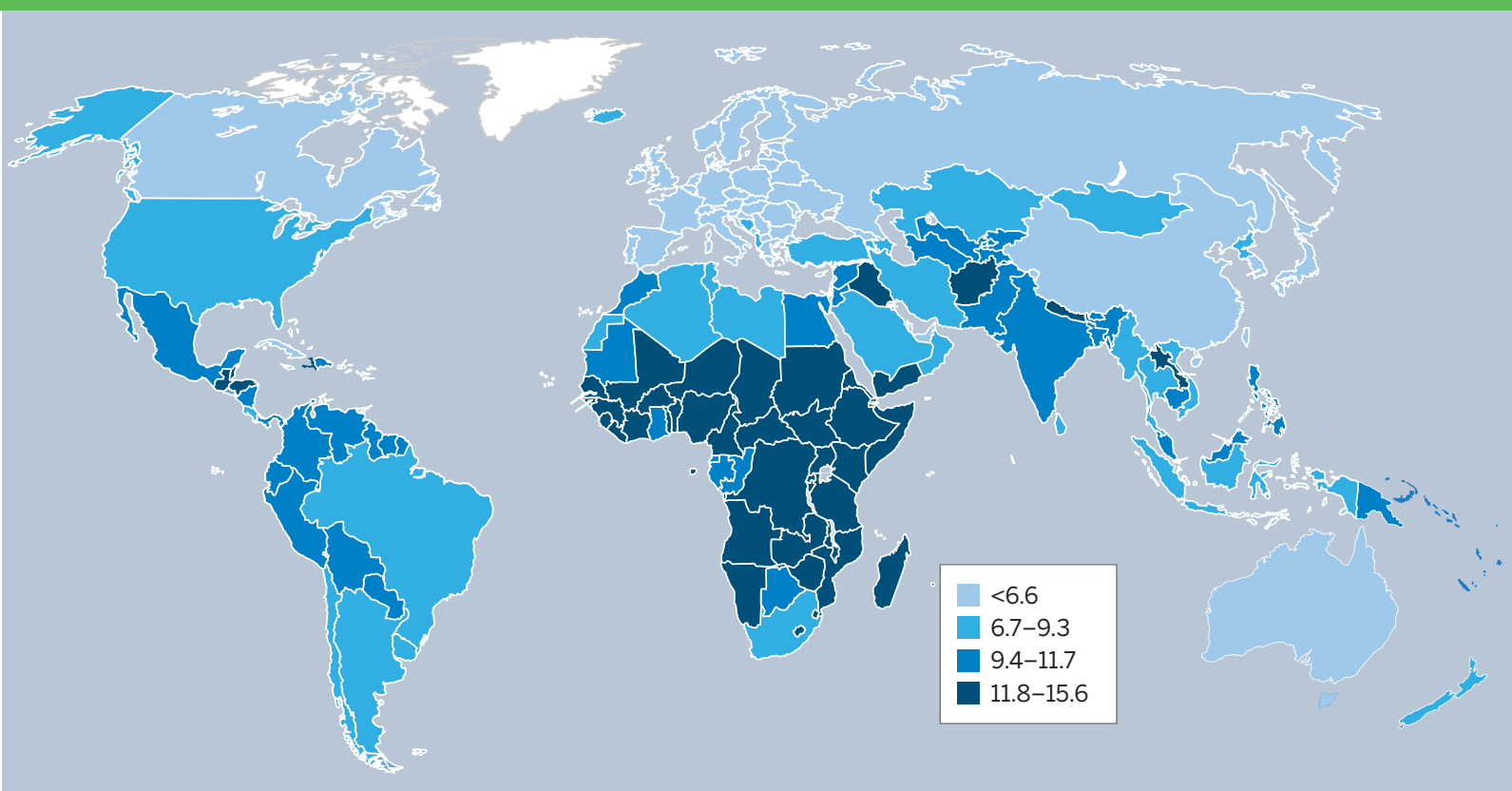

Remarque: Les catégories de la légende reflètent les quartiles de la répartition globale. Source: UNDESA (2013). Analyse réalisée par Katharine McCarthy, Population Council. 


\section{Le début de l'adolescence est carac- térisé, de manière universelle, par les changements rapides et impor- tants qui apparaissent à la puberté, et influencent la santé physique, mentale et émotionnelle des jeunes.}

\section{Les étapes du développement de la jeune adolescente et du jeune adolescent}

La plupart des jeunes adolescents entrent dans la puberté au début de l'adolescence, les filles plus tôt que les garçons. Le début de la puberté marque un changement de vie brusque, en particulier pour les filles (Breinbauer et Maddaleno 2005; Patton et al. 2012; Sawyer et al. 2012). Certains jeunes adolescents auront également leur premier rapport sexuel au début de l'adolescence. Les filles (et certains garçons) sont particulièrement vulnérables à la coercition sexuelle au début de l'adolescence (Jejeebhoy, Shah et Thapa 2005; UNICEF 2014c). Les autres transitions qui, dans certains contextes, accompagnent la puberté comprennent le fait de quitter l'école; d'entrer sur le marché du travail; le mariage d'enfants; et le fait de commencer à s'occuper d'enfants, de devenir parent ou de travailler. Dans de nombreux pays, ces actions peuvent entraîner à leur tour une exposition des adolescents au VIH, à la privation matérielle, aux conflits politiques et sociaux, ce qui érode les filets de sécurité traditionnels et augmente la vulnérabilité.

Le début de l'adolescence est caractérisé, de manière universelle, par les changements rapides et importants qui apparaissent à la puberté, et influencent la santé physique, mentale et émotionnelle des jeunes (Sawyer et al. 2012; Igras et al. 2014). En effet, de nombreux responsables de la mise en œuvre des programmes et chercheurs adoptent une perspective écologique et basée sur le parcours de vie qui examine les effets multiples et l'interdépendance des éléments sociaux sur les personnes alors qu'elles progressent à travers cette phase de leur vie (Blum et al. 2012; Blum, Astone et Decker 2013). (Voir la Section 4.)

Par conséquent, la plupart des scientifiques et des praticiens perçoivent ces années comme une série d'étapes plutôt que comme une étape homogène. L'Organisation panaméricaine de la santé (OPS) a proposé un modèle global du développement des adolescents, qui intègre des théories diverses sur le développement ainsi que des recherches sur l'impact de l'environnement, la maturation reproductive et le traitement cognitif et de l'information. (Voir la classification des étapes de l'adolescence établie par l'OPS, Figure 3.)

Dans ce système de classification, qui commence à la préadolescence et se termine au début de l'âge adulte, les changements sont groupés en cinq domaines de développement (Tableau1): les changements du corps, du cerveau, sexuels,

\section{FIGURE 3 Les étapes de l'adolescence (classification de l'OPS)}

\begin{tabular}{|c|c|c|c|c|c|c|c|c|c|c|c|c|c|c|c|}
\hline Âge & 9 & 10 & 11 & 12 & 13 & 1 & 4 & 16 & 17 & 18 & 19 & 20 & 22 & 23 & 24 \\
\hline FILLES & \multicolumn{3}{|c|}{ PRÉADOLESCENCE } & \multicolumn{3}{|c|}{$\begin{array}{c}\text { DÉBUT DE } \\
\text { L'ADOLESCENCE }\end{array}$} & \multicolumn{2}{|c|}{$\begin{array}{c}\text { MILIEU DE } \\
\text { L'ADOLESCENCE }\end{array}$} & \multicolumn{2}{|c|}{$\begin{array}{c}\text { FIN DE } \\
\text { L'ADOLESCENCE }\end{array}$} & \multicolumn{2}{|c|}{ JEUNESSE } & \multicolumn{3}{|c|}{ DEBUT DE L'ÂGE ADULTE } \\
\hline GARÇONS & & \multicolumn{3}{|c|}{ PRÉADOLESCENCE } & \multicolumn{3}{|c|}{$\begin{array}{c}\text { DÉBUT DE } \\
\text { L'ADOLESCENCE }\end{array}$} & \multicolumn{2}{|c|}{$\begin{array}{c}\text { MILIEU DE } \\
\text { L'ADOLESCENCE }\end{array}$} & $\begin{array}{c}\text { FIN DE } \\
\text { ADOLESC. }\end{array}$ & \multicolumn{2}{|c|}{ JEUNESSE } & \multicolumn{3}{|c|}{ DEBUT DE L'ÂGE ADULTE } \\
\hline & \multicolumn{6}{|c|}{$\begin{array}{l}\text { Les domaines de } \\
\text { changements qui se } \\
\text { produisent entre l'âge de } \\
10 \text { et de } 14 \text { ans, différenciés } \\
\text { par sexe, comprennent: }\end{array}$} & \multicolumn{9}{|c|}{$\begin{array}{l}\text { - LE DÉVELOPPEMENT PHYSIQUE } \\
\text { - LES CHANGEMENTS BIOLOGIQUES (début de la puberté) } \\
\text { - LE DÉVELOPPEMENT COGNITIF ET ÉMOTIONNEL } \\
\text { - L'ÉVOLUTION DES ROLES SOCIAUX (chez les plus pauvres, } \\
\text { un changement radical vers des responsabilités d'adulte) }\end{array}$} \\
\hline
\end{tabular}

Source: Adapté de Breinbauer et Maddaleno (2005) 
émotionnels et sociaux. Bien que d'autres définissent ces domaines de manière quelque peu différente ou les regroupent en changements physiques, cognitifs et socio-émotionnels (Sawyer et al. 2012), les éléments fondamentaux demeurent les mêmes. Puisque ce rapport met l'accent sur les très jeunes adolescents, seuls les changements s'appliquant à la préadolescence et au début de l'adolescence sont inclus ici. Nous avons choisi cette classification particulière car elle décompose les domaines en fonction de l'âge et du genre, qui sont des distinctions particulièrement importantes pendant l'adolescence. Les fourchettes d'âge données sont des estimations moyennes de l'âge auquel les changements développementaux se produisent. II existe des exceptions à ces limites d'âge, avec les adolescents précoces à une extrémité du spectre et les

TABLEAU 1 Les étapes du développement des très jeunes adolescents, par âge et par genre

\section{DOMAINES DE DÉVELOPPEMENT

LES
CHANGEMENTS
DU CORPS \\ LES \\ CHANGEMENTS DU CERVEAU}

\section{CHANGEMENTS} SEXUELS

\section{CHANGEMENTS} ÉMOTIONNELS

CHANGEMENTS SOCIAUX

\section{LA PRÉADOLESCENCE (de 9 à 12 ans chez les filles, de 10 à 13 ans chez les garçons)}

La poussée de croissance commence; le corps acquiert progressivement des caractéristiques sexuelles secondaires. Augmentation des graisses corporelles et du poids. Une augmentation progressive de la recherche de sensations fortes.

Un changement progressif se produit de la pensée égocentrique à la pensée sociocentrique. Un raisonnement logique plus concret. Une soif croissante d'informations nouvelles, mais le langage reste concret. Peu de développement du lobe préfrontal et des fonctions exécutives.

Les garçons et les filles explorent des rôles masculins et féminins plus différenciés. Pour les filles, l'androgynie est une alternative viable à la féminité exclusive, tandis que pour les garçons, la masculinité exclusive correspond aux attentes sociales.

Augmentation progressive de la timidité, avec des fluctuations de l'image de soi et des sentiments d'embarras. Besoin naissant d'une plus grande intimité, d'individuation et davantage d'autonomie émotionnelle vis-à-vis des parents. Capacité croissante à différencier les nuances et degrés des sentiments.

Le besoin d'un meilleur ami ou d'une meilleure amie du même sexe apparaît. Le temps est encore passé avec les parents et la supervision parentale est toujours présente, mais ils commencent à diminuer, accompagnés par une augmentation progressive des conflits entre le préadolescent et les parents. La sensibilité à la pression exercée par les pairs augmente.
LE DÉBUT DE L'ADOLESCENCE (de 12 à 14 ans chez les filles, de 13 à 15 ans chez les garçons)

Début des menstruations pour les filles (âge moyen=12,4 ans), et de l'éjaculation pour les garçons (âge moyen=13,4 ans). Une poussée de croissance importante et une augmentation marquée de la recherche de sensations fortes, en particulier chez les garçons.

Une pensée plus abstraite. II y a toujours peu de développement du lobe préfrontal et des fonctions exécutives (par ex., la prise de décisions, le jugement et la résolution des problèmes), en particulier chez les garçons. Approfondissement de la réflexion morale.

L'excitation sexuelle augmente, ainsi que le besoin de masturbation. D'autres comportements autoérotiques se produisent, comme les fantasmes sexuels et les éjaculations nocturnes. L'identité sexuelle se développe au cours des premières années de la vie, mais est de plus en plus mise à l'épreuve; développement de l'orientation sexuelle.

Niveau de timidité élevé et fluctuations importantes de l'image de soi. Le niveau de stress augmente, en particulier chez les filles. Besoin d'une autonomie émotionnelle plus importante vis-à-vis des parents; désidéalisation des parents, définition des opinions personnelles de l'adolescent. Augmentation de la dépendance émotionnelle à l'égard des amis. L'intimité, la loyauté, et les valeurs et attitudes partagées prennent une plus grande importance dans l'amitié.

Davantage de temps est passé avec les sous-groupes sociaux (la bande) et/ou seul. Intérêt naissant pour le sexe opposé et/ou des amis «différents». Moins de temps passé avec les parents, la supervision parentale diminue, et les conflits à propos de l'indépendance augmentent. De nouveaux privilèges sociaux sont attendus. La sensibilité à la pression exercée par les pairs atteint un sommet.

Source: Adapté de Breinbauer et Maddaleno (2005). 
Le début de l'adolescence est un

moment crucial pendant lequel

l'exposition aux influences négatives

et la perte de droits peuvent

entraîner les vies sur une mauvaise

voie, même si parfois les effets de

ces expositions nocives sur la santé

ne se manifestent que plus tard au cours de la vie.

adolescents ayant une maturité tardive à l'autre. On estime que l'âge du début de la puberté a chuté au cours de ces dernières décennies, bien que les données soient plus claires pour les filles, ce qui allonge potentiellement la période de vulnérabilité (Bellis, Downing et Ashton 2006; Herman-Giddens 2006; Downing et Bellis 2009). La rapidité avec laquelle un jeune traverse ces étapes dépend de nombreux facteurs endogènes et environnementaux, notamment les différences biologiques; les capacités cognitives; les expériences éducatives, émotionnelles et de la vie; et les contextes culturels et sociaux. Puiser des connaissances scientifiques à partir des sciences sociales, cognitives et comportementales et intégrer des domaines de la biologie au modèle peut offrir une compréhension plus complexe de cette étape de la vie, qui peut ensuite avoir une incidence sur la conception des programmes destinés auxTJA.

\section{Le développement du cerveau pendant l'adolescence}

Il est largement admis que l'adolescence est une période de changements marqués dans la structure et la fonction du cerveau (Blakemore 2008; Steinberg 2014), et que le développement du cerveau se poursuit tout au long de l'adolescence. Le cerveau est le dernier organe du corps à atteindre sa pleine maturité, et les estimations actuelles indiquent que ce processus n'est probablement pas achevé avant la fin de la vingtaine et le début de la trentaine (Blakemore 2008; Giedd 2008; Dosenbach et al. 2010; Steinberg 2014). Cela signifie que pendant une grande partie de l'adolescence, le cerveau est en mode «construction ». Les neurones communiquent entre eux par le biais des synapses, qui sont très excitables et «plastiques » vers la fin de l'enfance et pendant l'adolescence. Les synapses se développent lorsqu'elles sont utilisées fréquemment; l'entraînement peut donc aider à maîtriser la mémorisation, les compétences musicales et les performances sportives: des domaines dans lesquels les jeunes adolescents réussissent souvent. C'est cette programmation biologique du cerveau qui facilite l'apprentissage, le fait de se souvenir et le développement de certaines compétences pendant l'adolescence, par rapport à l'âge adulte.

En même temps, cette plasticité neuronale pourrait également être à l'origine de l'addiction. Comme c'est le cas pendant l'apprentissage, les synapses sont activées par des substances qui entraînent un renforcement des circuits de récompense, entraînant à son tour un état d'addiction. Certains des «stimuli» négatifs les plus courants rencontrés par les adolescents dont il a été prouvé qu'ils modifient le développement du cerveau comprennent certaines drogues, la nicotine, l'alcool ainsi que les facteurs environnementaux tels que le stress, la surcharge numérique et le manque de sommeil (Jensen et Nutt 2015). II convient cependant de noter que les adolescents réagissent davantage à la récompense qu'aux stimuli négatifs ou punitifs.

Les cortex frontal et préfrontal sont les régions du cerveau responsables du jugement, du raisonnement, du contrôle des impulsions et de l'empathie. L'immaturité du cortex frontal explique, en partie, les comportements à risque adoptés par les adolescents, mais les processus cognitifs, affectifs et sociaux jouent également un rôle clé. Le contexte social influence également les hormones endogènes dans le corps, la manière dont le système nerveux se développe et dont les adolescents prennent des décisions (Crone et Dahl 2012; Blakemore et Mills 2014).

L'étude du développement du cerveau adolescent a réalisé d'énormes progrès en un laps de temps relativement court. Les informations dont nous disposons sur le fonctionnement du cerveau sont aujourd'hui plus nombreuses que jamais, et les adolescents d'aujourd'hui sont la première gé- 
nération de jeunes pour lesquels nous disposons d'informations si approfondies et importantes concernant leur développement. Utiliser au mieux ces informations pour concevoir des interventions qui prennent davantage en compte le développement cognitif et social des jeunes adolescents est un objectif qui en vaut la peine.

\section{Le début de l'adolescence : un moment décisif pour les filles}

Comme cela a été mentionné précédemment, à partir du début de la puberté, les trajectoires empruntées par les filles peuvent commencer à diverger considérablement de celles des garçons. Si aucun mécanisme de protection n'est en place, ces changements peuvent entraîner une perte de droits, qui peut influencer irrémédiablement la vie future des filles.

Dans certains PRFM, comme l'attestent les données issues de certains pays présentés dans la Figure 4, il existe une tendance générale selon laquelle les filles abandonnent l'école à un moment correspondant au début de la puberté
(Panneau A). Un exemple de filles indigènes âgées de10 à 19 ans au Guatemala illustre également la manière dont le fait de s'écarter dans un domaine (par ex., quitter l'école) peut coïncider avec d'autres transitions négatives (une grossesse précoce) (Panneau B). Ces changements, ainsi que l'isolement social qui y est souvent associé, peuvent précipiter d'autres événements qui ont une influence négative sur le bien-être et la santé future des jeunes filles, comme une grossesse précoce et le fait de contracter une infection sexuellement transmissible, par exemple le VIH (Hallman et al. 2007). Les données basées sur les déclarations d'adolescentes au Malawi confirment également cet enchaînement « d'événements négatifs dans la vie». En particulier, une étude a démontré que les filles déclarent vivre un nombre plus important d'événements généralement négatifs, comme quitter l'école, une initiation sexuelle précoce et le mariage d'enfants ( $42 \%$ des filles ont vécu trois événements de ce type ou plus) par rapport aux garçons (4\%) (Mensch et al. 2011; Mensch et al. 2014).

\section{FIGURE 4 Le début de l'adolescence : lorsque la vie des filles prend une mauvaise direction}

A. Étude de cas de quatre pays : Pourcentage de filles âgées de 10 à 17 ans allant à l'école, par âge

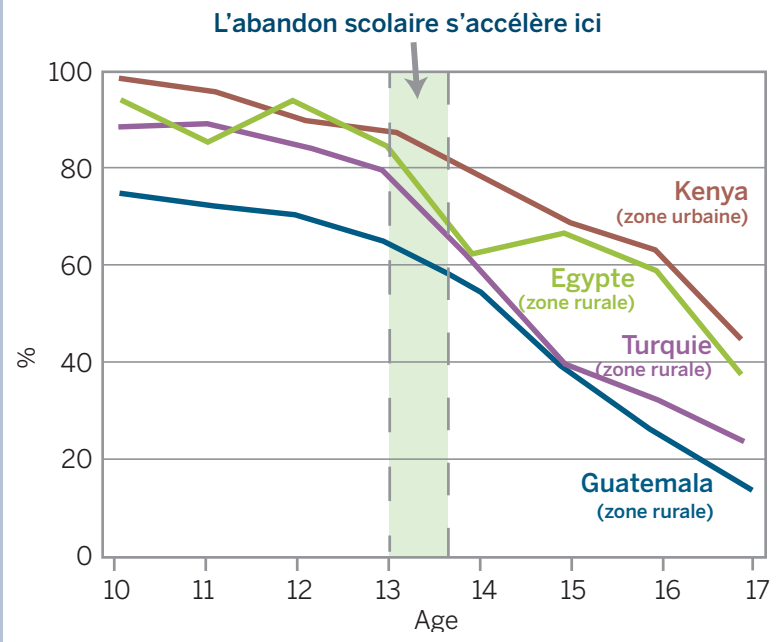

Sources: (Guatemala) Hallman et al. 2007; (Turquie 2003, Kenya 2003) Population Council Adolescent Experience In Depth Data Guides Series 2000-15; (Égypte) ERF 2006.
B. L'exemple du Guatemala : Scolarisation et début de la transition vers l'âge adulte chez les filles âgées de 10 à 19 ans

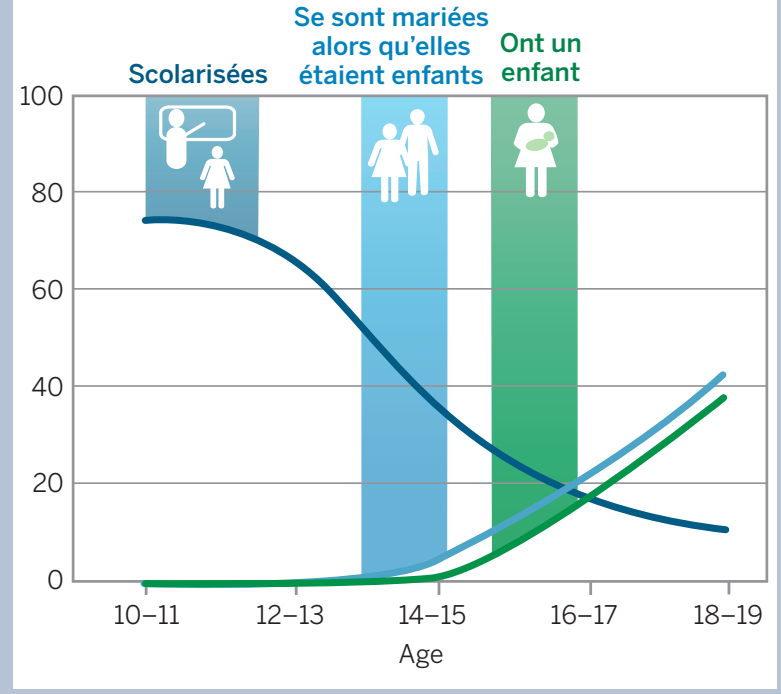

Source: Hallman et al. 2007. 

La santé et le

bien-être des TJA:

vue d'ensemble et état actuel
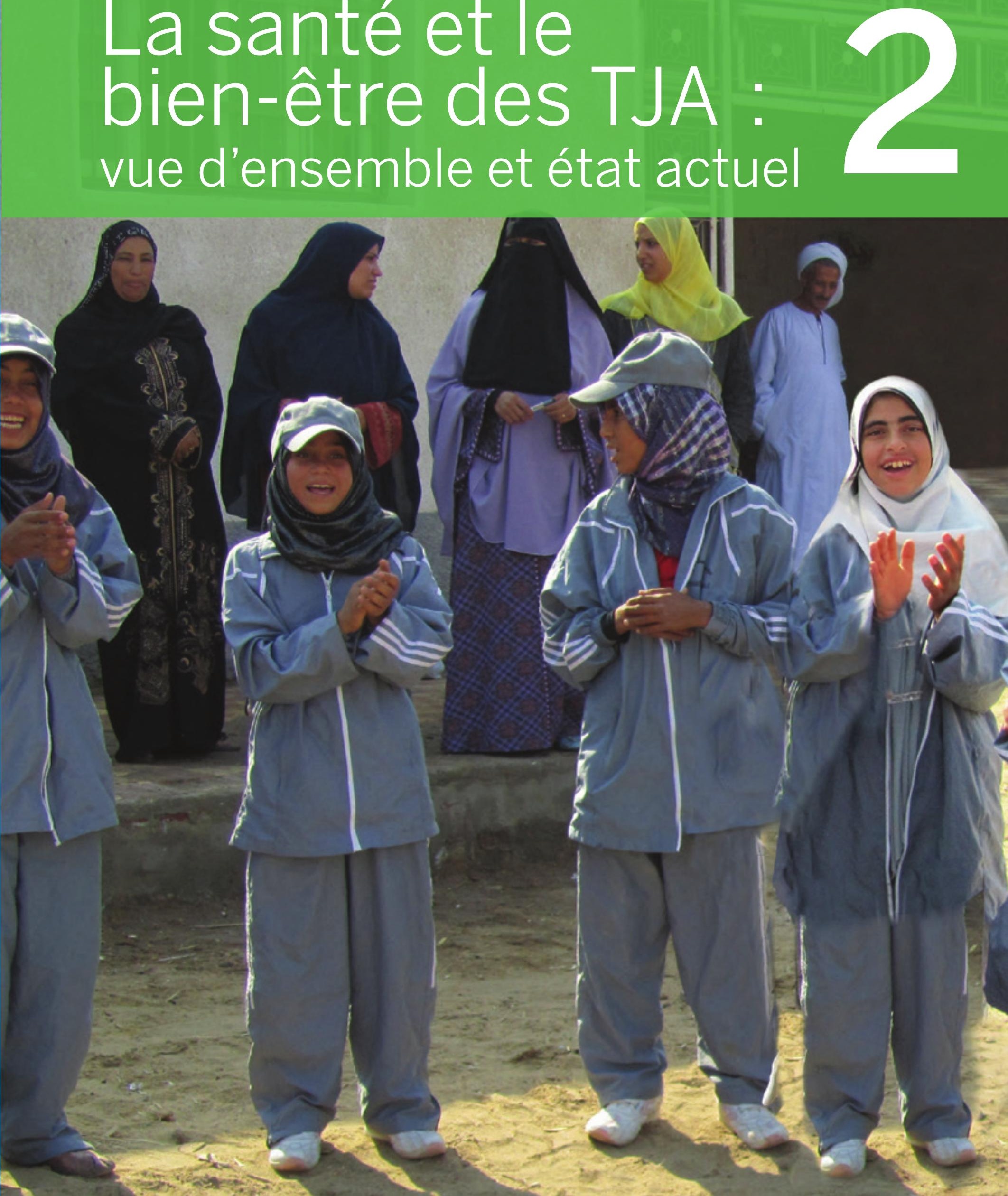


\subsection{La maladie et l'incapacité: comment s'en sortent les jeunes adolescentes et adolescents?}

Les problèmes de santé les plus courants pour les TJA sont chroniques plutôt que des maladies mettant la vie en danger. En matière de morbidité et de mortalité, une fois que les filles et les garçons ont survécu aux cinq premières années de la vie, ils bénéficient généralement d'une période de bonne santé relative, entre les dangers de la jeune enfance et le début des risques et des problèmes de santé liés à l'âge adulte. Cependant, le fait de souffrir de problèmes de santé au moment de ces changements physiques et sociaux si dynamiques peut avoir des effets durables. Nous étudions les transitions épidémiologiques qui ont lieu au début de l'adolescence et discutons de leur influence sur la santé immédiate et plus tard au cours de la vie.

\section{Données mondiales sur la maladie et l'incapacité}

Les taux de mortalité des 10-14 ans sont faibles par rapport aux autres phases de la vie et ont suivi une tendance légèrement à la baisse au cours de ces dernières décennies. Bien que les taux de mortalité soient faibles, les cinq premières causes de décès chez les TJA sont le VIH et le SIDA, les maladies diarrhéiques, les accidents de la route, les infections des voies respiratoires inférieures et la noyade (OMS 2013; OMS 2014a et 2014b). À ce stade de la vie, les taux de mortalité sont similaires pour les filles et les garçons. Par exemple, en2012, le taux de mortalité due au VIH/SIDA était sensiblement équivalent chez les deux sexes. Même s'il existe des variations pour les autres causes de décès (par exemple, les maladies diarrhéiques sont une cause plus importante de mortalité chez les filles, tandis que les taux d'accidents de la route sont plus élevés chez les garçons), ces écarts sont limités compte tenu de la prévalence relativement faible de la maladie.
Malgré les nombreuses similarités concernant les causes de mortalité chez les TJA, il existe de grandes différences concernant les régions où ces décès surviennent: 61 décès de TJA sur 100 se sont produits en Afrique subsaharienne et en Méditerranée orientale (OMS 2013; OMS 2014b).

Les problèmes de santé chroniques non mortels, comme les troubles de santé mentale, sont une autre cause importante de mauvaise santé ou de morbidité chez les TJA. Lorsqu'elles ne sont pas identifiées et traitées, ces maladies chroniques peuvent également représenter un obstacle à une transition saine vers l'âge adulte.

Comprendre la morbidité est important pour orienter l'établissement des priorités en matière de santé publique. Les données de morbidité permettent d'évaluer les maladies et conditions non mortelles qui se développent pendant l'adolescence et peuvent avoir des répercussions tout au long de la vie. Les années de vie corrigées de l'incapacité (AVCI) sont l'une des manières de mesurer la morbidité. L'AVCl est un indicateur de la charge globale de morbidité et mesure les années de vie saine perdues en raison d'une mauvaise santé, d'une incapacité ou d'un décès prématuré.

En2012, les cinq premières causes d'AVCI chez les 10-14 ans à l'échelle mondiale étaient les troubles dépressifs, l'anémie ferriprive, le VIH/ SIDA, les maladies diarrhéiques et les accidents de la route (OMS 2014a). Bien qu'il existe des différences entre les premières causes d'AVCI pour les filles et pour les garçons âgés de 10 à 14 ans (par exemple, la dépression est une source plus importante de morbidité chez les filles, tandis que les accidents de la route sont plus importants chez les garçons), les différences se manifestent plus clairement chez les 15-19 ans. Comme pour les différences régionales en matière de mortalité pour les TJA, la région africaine représente le niveau le plus élevé d'AVCI chez les 10-14 ans, suivie par la région de la Méditerranée orientale (OMS 2014b). 


\subsection{Comprendre les} comportements des TJA en matière de santé pour endiguer la propagation des maladies non transmissibles

Les années de vie corrigées de l'incapacité ne reflètent pas la charge de morbidité future due aux comportements de santé qui commencent ou sont renforcés pendant l'adolescence (Gore et al. 2011). Par exemple, même si certains comportements comme le fait de fumer, de consommer de l'alcool et les pratiques alimentaires malsaines, peuvent commencer pendant l'adolescence, ces comportements à risque n'entraînent souvent pas de maladies non transmissibles (MNT), comme les maladies cardiovasculaires, les maladies respiratoires, le diabète et le cancer, avant l'âge adulte. L'Organisation mondiale de la Santé estime que $70 \%$ de l'ensemble des décès prématurés sont attribuables aux comportements nuisibles pour la santé établis pendant l'adolescence, et les MNT sont les principales causes de mortalité pour les adultes à l'échelle mondiale, représentant environ 38 millions de décès par an (dont 28 millions dans les PRFM) (PRB 2015).

De plus, d'après les prévisions, dans certaines régions des PRFM connaissant une mondialisation et une modernisation rapides, la consommation d'alcool, de tabac et les habitudes alimentaires nuisibles pour la santé devraient augmenter chez les jeunes. Étant donné que les TJA représentent une grande partie de la population dans de nombreux pays (voir la Figure2), les MNT devraient devenir une cause majeure de décès dans l'ensemble de l'Afrique (PRB 2015). Le coût élevé associé aux MNT dans ces contextes disposant déjà de ressources limitées a des conséquences majeures sur la capacité des pays à traiter et répondre à ces maladies. Pour résumer, le début de l'adolescence représente une occasion unique pour les interventions qui visent à améliorer la santé future des personnes et à influencer les trajectoires des épidémies futures.
En 2012, les cinq premières causes d'AVCI chez les 10-14 ans à l'échelle mondiale étaient les troubles dépressifs, l'anémie ferriprive, le VIH/SIDA, les maladies diarrhéiques et les accidents de la route.

\subsection{Les résultats de santé pour les TJA: le monde divergent des filles et des garçons}

Nous abordons ensuite trois résultats importants sur la santé des TJA, compte tenu de leur influence durable et potentiellement irrémédiable: l'activité sexuelle et la santé reproductive, la santé mentale et la nutrition.

\section{Activité sexuelle, santé reproductive, mariages d'enfants : des conséquences profondément négatives pour les filles}

Les engagements des politiques et des programmes pour répondre aux besoins des adolescents en matière de santé sexuelle et reproductive (SSR) se sont étendus grâce à l'attention et aux investissements accrus liés à la santé des adolescents depuis le début du21e siècle. Cependant, les données sur les connaissances, les attitudes, les comportements et les résultats en matière de SSR chez les filles et les garçons de 10 à 14 ans sont extrêmement limitées, en raison des sensibilités liées à la collecte de telles informations auprès de ce groupe d'âge. En revanche, nous savons que les jeunes adolescentes et adolescents du monde entier sont généralement peu ou mal informés concernant leur corps, leur santé sexuelle et reproductive et les comportements favorables à la santé (Santhya et Jejeebhoy 2015). Les fausses informations abondent concernant la fertilité (les premières menstruations et l'éjaculation), la sexualité, la conception, la contraception, etc. (Bankole et al. 2007; Dixon-Mueller 2010;

Igras et al. 2014), ce qui peut entraver l'adoption de comportements favorables à la santé. 
Lorsqu'il existe des données sur la santé sexuelle et reproductive des jeunes adolescents, il y a davantage de données disponibles pour les filles que pour les garçons, pour les 15-19 ans que pour les 10-14 ans, et pour les filles mariées que pour les filles non mariées (UNICEF 2014a). Lorsque nous observons les données disponibles pour les 15-19 ans, I'UNICEF rapporte qu'environ $11 \%$ des filles et $6 \%$ des garçons âgés de 15 à 19 ans dans le monde en développement, à l'exception de la Chine, ont eu des rapports sexuels avant l'âge de 15 ans (UNICEF 2011). II convient cependant de noter que, d'après ces données, la prévalence rapportée pour les filles est près de deux fois supérieure à celle concernant les garçons. L'analyse des données de l'enquête démographique et de santé (EDS) (se référer au Tableau2) indique qu'une activité sexuelle précoce est assez courante dans certaines régions du monde; $15 \%$ ou plus des filles âgées de 15 à 19 ans ont indiqué avoir eu des rapports sexuels avant l'âge de 15 ans dans 10 pays d'Afrique subsaharienne.

L'activité sexuelle précoce, en particulier dans des circonstances de rapports sexuels forcés, augmente le risque d'exposition aux infections sexuellement transmissibles, comme le VIH. Bien que la prévalence du $\mathrm{VIH}$ soit généralement faible chez les 10-14 ans et qu'il y ait peu de différence entre les garçons et les filles (Idele et al. 2014), cela change plus tard au cours de l'adolescence. Chez les adolescents âgés de 15 à 19 ans, les filles commencent à connaître des taux de VIH plus élevés que les garçons; environ deux tiers des nouvelles infections par le VIH chez les adolescents âgés de 15 à 19 concernent les filles (UNICEF 2013). Bien que les taux de prévalence varient en fonction des pays, cette tendance à la féminisation du VIH est valable dans la majeure partie de l'Afrique subsaharienne où la transmission hétérosexuelle est la plus élevée (Idele et al. 2014).

Les autres menaces à la santé reproductive peuvent inclure des rites culturels de passage qui coïncident souvent avec la puberté et donc, avec le début de l'adolescence. Dans certains contextes, ces rites de passage comprennent des pratiques traditionnelles nocives pour les jeunes filles, comme les mutilations génitales féminines (MGF) et les excisions génitales féminines (EGF). L'E/MGF implique l'ablation partielle ou totale des organes génitaux de la femme, et est pratiquée dans de nombreux pays d'Afrique, ainsi que dans plusieurs pays du Moyen-Orient et d'Asie (UNICEF 2014b). Une étude de2013 estime que 14,1 millions de filles âgées entre 10 et 14 ans dans 27 pays d'Afrique, et y compris le Yémen, sont susceptibles de subir une excision (Yoder, Wang et Johansen 2013).

Une partie des jeunes adolescentes sont mariées, et l'activité sexuelle a lieu dans le contexte du mariage. Dans 5PRFM sur 71, les grossesses ayant lieu avant l'âge de 15 ans ont été rapportées par $5 \%$ ou plus des filles âgées de 15 à 19 ans (voir le Tableau2). Bien que ces chiffres indiquent que les grossesses précoces ne sont pas courantes, ils indiquent aussi qu'une partie des filles courent toujours un risque et ont besoin de services (se référer à la Section 4.2 pour une discussion sur les programmes de SSR pour les TJA).

\section{Le mariage d'enfants : une violation des droits humains}

Dans certains cas, la transition précoce vers l'activité sexuelle et la maternité se produit dans le cadre de mariages d'enfants. On reconnaît de plus en plus que le mariage d'enfants est une violation des droits humains des filles et un obstacle pour les résultats globaux de développement. Le mariage d'enfants (défini comme ayant lieu avant l'âge de18ans) coïncide avec l'activité sexuelle et le commencement de la grossesse, et impose aux filles des rôles et responsabilités auxquels elles ne sont pas préparées.

Des recherches ont rapporté des effets négatifs chez les filles qui se marient tôt, bien que le lien de cause à effet n'ait pas encore été établi (Mensch, Singh et Casterline 2005). Par exemple, des études ont révélé que les filles qui se marient avant l'âge de 18 ans connaissent des taux supérieurs de violences conjugales que celles qui ne sont pas mariées (UNICEF 2005; UNICEF 2014b), sont plus susceptibles d'abandonner l'école (Erulkar et al. 2004), vivent dans des ménages plus pauvres (UNICEF 2005), courent un risque plus élevé de contracter des infections sexuellement transmissibles, dont le VIH/SIDA (Clark 2004), et ont plus 


\begin{tabular}{|c|c|c|c|c|c|c|c|c|c|}
\hline PAYS & $\begin{array}{l}\text { ANNÉE DE } \\
\text { LENQUEETE } \\
\text { EDS LA } \\
\text { PLUS } \\
\text { RÉCENTE }\end{array}$ & $\begin{array}{l}\text { \% AYANTEU } \\
\text { RAPPORTS } \\
\text { SEXUELS } \\
\text { AVANT } \\
\text { L'ÂGE DE } \\
15 \text { ANS }\end{array}$ & $\begin{array}{l}\% \\
\text { MARIÉES } \\
\text { ANANT } \\
\text { L'ÂGE DE } \\
15 \text { ANS }\end{array}$ & $\begin{array}{l}\text { \% AYANT } \\
\text { DONNÉ } \\
\text { NAISSANCE } \\
\text { AVANT } \\
\text { L'ÂGE DE } \\
15 \text { ANS }\end{array}$ & PAYS & $\begin{array}{l}\text { ANNÉE DE } \\
\text { L'ENQUÊTE } \\
\text { EDS LA } \\
\text { PLUS } \\
\text { RÉCENTE }\end{array}$ & $\begin{array}{l}\text { \% AYANT EU } \\
\text { RAPPORTS } \\
\text { SEXUELS } \\
\text { AVANT } \\
\text { L'ÂGE DE } \\
15 \text { ANS }\end{array}$ & $\begin{array}{l}\% \\
\text { MARIÉES } \\
\text { AVANT } \\
\text { L'ÂGE DE } \\
15 \text { ANS }\end{array}$ & $\begin{array}{l}\text { \% AYANT } \\
\text { DONNÉ } \\
\text { NAISSANCE } \\
\text { AVANT } \\
\text { L'ÁGE DE } \\
15 \text { ANS }\end{array}$ \\
\hline \multicolumn{5}{|c|}{ Afrique occidentale et centrale } & \multicolumn{5}{|c|}{ Amérique latine et Caraïbes } \\
\hline Bénin & 2006 & 10 & 5 & 2 & Bolivie & 2008 & 7 & 3 & 1 \\
\hline Burkina Faso & 2010 & 3 & 6 & 1 & Brésil & 1996 & 11 & 4 & 1 \\
\hline Burundi & 2011 & 3 & 1 & 0 & Colombie & 2010 & 13 & 4 & 2 \\
\hline Cameroun & 2011 & 9 & 9 & 3 & Guatemala & 1999 & 7 & 7 & 2 \\
\hline Congo & 2006 & 23 & 4 & 2 & Guyana & 2009 & 9 & 5 & 2 \\
\hline Côte d'Ivoire & 2012 & 18 & 6 & 4 & Haïti & 2012 & 14 & 3 & 1 \\
\hline Gabon & 2012 & 16 & 4 & 3 & Honduras & 2012 & 11 & 8 & 2 \\
\hline Ghana & 2008 & 8 & 3 & 1 & Nicaragua & 2001 & 9 & 9 & 2 \\
\hline Guinée & 2012 & 12 & 14 & 6 & Paraguay & 1990 & 5 & 3 & 1 \\
\hline Liberia & 2013 & 22 & 4 & 3 & Pérou & 2008 & 5 & 2 & 1 \\
\hline Mali & 2013 & 14 & 19 & 8 & Répub. dominicaine & 2007 & 12 & 10 & 2 \\
\hline Mauritanie & 2001 & 2 & 13 & 4 & \multicolumn{5}{|c|}{ Asie du sud et du sud-est } \\
\hline Niger & 2012 & 8 & 24 & 5 & Bangladesha $^{a}$ & 2011 & 1 & 17 & 4 \\
\hline Nigeria & 2013 & 5 & 12 & 2 & Cambodge & 2011 & 0 & 1 & 0 \\
\hline Répub. centrafricaine & 1995 & 23 & 16 & 5 & Inde & 2006 & 1 & 8 & 1 \\
\hline Répub. dém. du Congo & 2013 & 17 & 6 & 3 & Indonésie & 2012 & 1 & 2 & 0 \\
\hline Sao Tomé-et-Principe & 2009 & 9 & 3 & 1 & Maldives $^{a}$ & 2009 & 0 & 0 & 0 \\
\hline Sénégal & 2011 & 4 & 9 & 3 & Népal & 2011 & 3 & 5 & 0 \\
\hline Sierra Leone & 2013 & 17 & 6 & 4 & Pakistan ${ }^{a}$ & 2012 & n.d. & 2 & 0 \\
\hline Tchad & 2004 & 7 & 18 & 6 & Philippines & 2013 & 2 & 2 & 0 \\
\hline \multicolumn{5}{|c|}{ Afrique orientale et australe } & Timor-Leste & 2010 & 1 & 1 & 0 \\
\hline Afrique du Sud & 1998 & 8 & 0 & 1 & \multirow{2}{*}{\multicolumn{5}{|c|}{ Moyen-orient et Afrique du nord }} \\
\hline Comores & 2012 & 4 & 6 & 1 & & & & & \\
\hline Éthiopie & 2011 & 2 & 8 & 1 & Egypte ${ }^{a}$ & 2008 & n.d. & 1 & 0 \\
\hline Kenya & 2009 & 11 & 1 & 1 & Jordanie ${ }^{a}$ & 2012 & 0 & 0 & 0 \\
\hline Lesotho & 2010 & 8 & 1 & 1 & Maroc & 2004 & n.d. & 2 & 0 \\
\hline Madagascar & 2009 & 15 & 12 & 1 & Yémena & 1992 & n.d. & 9 & 2 \\
\hline Malawi & 2010 & 11 & 4 & 1 & \multicolumn{5}{|c|}{ Europe et républiques d’Asie centrale } \\
\hline Mozambique & 2011 & 22 & 10 & + & Albanie & 2009 & 1 & 1 & 0 \\
\hline Namibie & 2007 & $2<$ & 1 & 4 & Arménie & 2005 & 0 & 0 & 0 \\
\hline Ouganda & 2011 & $r^{\prime}$ & \pm & 1 & Azerbaïdjan & 2006 & 0 & 0 & 0 \\
\hline Rwanda & 2011 & 5 & 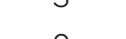 & 2 & Moldavie & 2005 & 1 & 1 & 0 \\
\hline Swaziland & 2007 & J & 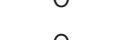 & 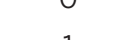 & Kazakhstan & 1999 & 1 & 0 & 0 \\
\hline Tanzanie & 2010 & & & & Ouzbékistan & 1996 & 0 & 1 & 0 \\
\hline Togo & 1998 & 10 & 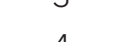 & $\perp$ & République kirghize & 2012 & 0 & 0 & 0 \\
\hline Zambie & 2007 & 11 & 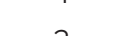 & 2 & Tadjikistan & 2012 & 0 & 0 & 0 \\
\hline Zimbabwe & 2011 & 4 & 3 & 1 & Turquie ${ }^{a}$ & 2004 & n.d. & 1 & 0 \\
\hline & & & & & Ukraine & 2007 & 1 & 0 & 0 \\
\hline
\end{tabular}

n.d. = Les données pour l'indicateur n'ont pas été recueillies. Remarque: Toutes les analyses sont basées sur des données des enquêtes démographiques et de santé. a Les femmes âgées entre 15 et 49 ans ayant déjà été mariées ont été interrogées. Les données pour les $15-$ 19 ans ont été ajustées pour représenter toutes les femmes du groupe d'âge. On suppose que les femmes qui n'ont jamais été mariées n'ont jamais donné naissance ou n'ont jamais eu de rapports sexuels, conformément aux normes EDS pour l'analyse et l'établissement de rapports. 
Bien que, globalement, la plupart des filles âgées entre 10 et 14 ans ne soient pas mariées, dans certains pays et régions, la proportion de filles âgées entre 15 et 19 ans mariées avant l'âge de 15 ans est considérable.

d'enfants à un âge plus précoce (UNICEF 2014a). L'analyse des données EDS issues de 38PRFM a également démontré que les filles âgées de 15 à 19 ans ont un risque de décès pendant la grossesse ou l'accouchement de $28 \%$ supérieur à celui des filles âgées entre 20 et 24 ans (Blanc, Winfrey et Ross 2013).

Bien que, globalement, la plupart des filles âgées entre 10 et 14 ans ne soient pas mariées, dans certains pays et régions, la proportion de filles âgées entre 15 et 19 ans mariées avant l'âge de 15 ans est considérable. La comparaison entre l'analyse EDS publiée dans le rapport de 2006 Investing When It Counts (Chong, Hallman et Brady 2006) et l'analyse actualisée de ce rapport (Tableau 2), indique que le pourcentage de filles âgées entre 15 et 19 ans qui déclarent avoir été mariées avant l'âge de 15 ans semble relativement stable dans la plupart des pays.

Il existe, cependant, quelques exceptions notables. Par exemple, la prévalence du mariage d'enfants en Éthiopie a baissé, passant de $14 \%$ en 2000 à $8 \%$ en 2011. Au Bangladesh, 27\% des filles ont déclaré s'être mariées avant l'âge de 15 ans en 1999, un chiffre qui est passé à $17 \%$ en2011. II convient de noter que ces deux pays ont réalisé des investissements considérables dans les politiques et les programmes visant à garder les filles à l'école et à retarder l'âge du mariage. D'autres analyses des tendances concernant l'âge du mariage ont prouvé que davantage de progrès ont été accomplis concernant le mariage des enfants avant l'âge de 18 ans (Mensch, Singh et Casterline 2005; UNICEF 2014a).

\section{La santé mentale : la dépression représente la première cause d'incapacité chez les filles}

L'adolescence est le moment où de nombreux problèmes de santé mentale apparaissent; près de la moitié des troubles mentaux présents à vie (y compris la dépression, les troubles bipolaires, les troubles anxieux, les troubles du contrôle des impulsions et les troubles liés à la consommation de substances) commencent avant l'âge de 14 ans (Belfer 2008; Kessler et al. 2007).

L'interruption des processus de développement qui se déroulent normalement pendant l'adolescence due à l'apparition d'une maladie mentale, comme l'achèvement des études et la capacité à former des relations d'amitié et romantiques, peut avoir une influence négative sur la santé future d'un adolescent, en particulier si la maladie n'a pas été traitée ou identifiée (Sawyer et al. 2012).

À l'échelle mondiale, on estime que parmi les enfants et adolescents âgés de 17 ans ou moins jusqu'à un sur cinq connaît un problème de santé mentale débilitant au cours d'une année donnée (Kieling et al. 2011). Bien que la charge totale de morbidité chez les adolescents soit généralement faible, les troubles neuropsychiatriques1 représentent près de la moitié (45\%) de l'ensemble des incapacités liées à la santé entre l'âge de 10 et24 ans (Gore et al. 2011). ${ }^{2}$ Chez les 10-14 ans, la dépression est la principale cause d'incapacité liée à la santé chez les filles, et la troisième cause chez les garçons. Cet écart entre les sexes concernant la dépression s'accroît autour de l'âge de 15-19 ans, la dépression restant

1 Les troubles neuropsychiatriques comprennent: la dépression, les troubles bipolaires, les troubles anxieux, la schizophrénie, l'épilepsie, les troubles liés à la consommation d'alcool et de drogue et la démence, entre autres (Gore et al. 2011).

2 Données de l'analyse de Gore et al. tirée de l'étude de I'OMS sur la charge de morbidité dans le monde, qui incluait 194 États membres de l'OMS appartenant à différentes régions du monde et groupes de revenus. Pour une liste complète, veuillez consulter: http:// www.who.int/countries/fr/. 
la cause principale d'incapacité liée à la santé chez les filles et la quatrième chez les garçons (OMS 2014b). Le suicide, qui est étroitement lié à la dépression, est également une cause majeure de mortalité chez les adolescentes et les adolescents, en particulier à l'âge de 15-19 ans (11,7 décès pour 100000 adolescents de 15 à 19 ans pour chaque sexe, respectivement) (OMS 2012b). En général, les problèmes de santé mentale se concentrent chez ceux qui vivent dans la pauvreté ou qui subissent d'autres formes d'inégalités sociales (Patel et al. 2007; Kieling et al. 2011).

\section{Le problème de la sous- et surnutrition}

Les années entre 10 et 14 ans correspondent généralement à une forte poussée de croissance qui implique des gains importants de poids et de taille. Ce développement entraîne une augmentation du volume total de sang et de la masse musculaire et osseuse. La maturation des organes reproducteurs commence également autour de cet âge, et la plupart des filles ont leurs premières menstruations. En raison de ces changements, les garçons comme les filles ont des besoins accrus en micronutriments à ce stade. Une nutrition inadéquate peut interférer avec la capacité des adolescents à atteindre leur plein potentiel de croissance et peut affaiblir leur fonctionnement cognitif (Hermoso et al. 2011).

Comme mentionné précédemment, chez les 10-14 ans, l'anémie ferriprive est l'une des cinq principales causes d'AVCI. II s'agit du déficit nutritionnel le plus courant, représentant 3,4\% des AVCl chez les garçons, et 3,5\% chez les filles (Gore et al. 2011). L'anémie est particulièrement préoccupante chez les filles ayant un risque de grossesse, comme dans les régions où le mariage d'enfants est courant, en raison du risque de retard de croissance de la mère et du faible poids à la naissance du nourrisson (Black et al. 2013).

En plus de la carence en micronutriments, l'apport alimentaire insuffisant ainsi qu'excessif peut entraîner des conséquences sur la santé qui se manifestent plus tard au cours de la vie, dont les maladies cardiovasculaires, l'obésité, le

\section{Chez les 10-14 ans, la dépression est la principale cause d'incapacité liée à la santé chez les filles, et la troisième cause chez les garçons.}

diabète et l'ostéoporose (Gore et al. 2011; Black et al. 2013). L'introduction d'aliments à forte teneur énergétique et transformés dans les régions se modernisant rapidement, en parallèle avec une activité physique faible (Keino et al. 2014), contribue à la tendance à la hausse de l'obésité et du diabète observée dans de nombreux PRFM (se référer à la Section 2.2 sur les maladies non transmissibles). Des régions comme l'Afrique évoluent des zones caractérisées par l'insécurité alimentaire aux zones caractérisées à la fois par l'insécurité alimentaire et la surnutrition. Par conséquent, nous constatons maintenant des épidémies de faim et d'obésité au sein des mêmes contextes. Une nutrition adéquate étant nécessaire pour répondre aux besoins accrus liés au développement des TJA, doter les TJA des compétences nécessaires pour naviguer au sein de ces environnements revêt une importance croissante. 



\section{Influences sociales et contextuelles sur la santé : preuves et observations}
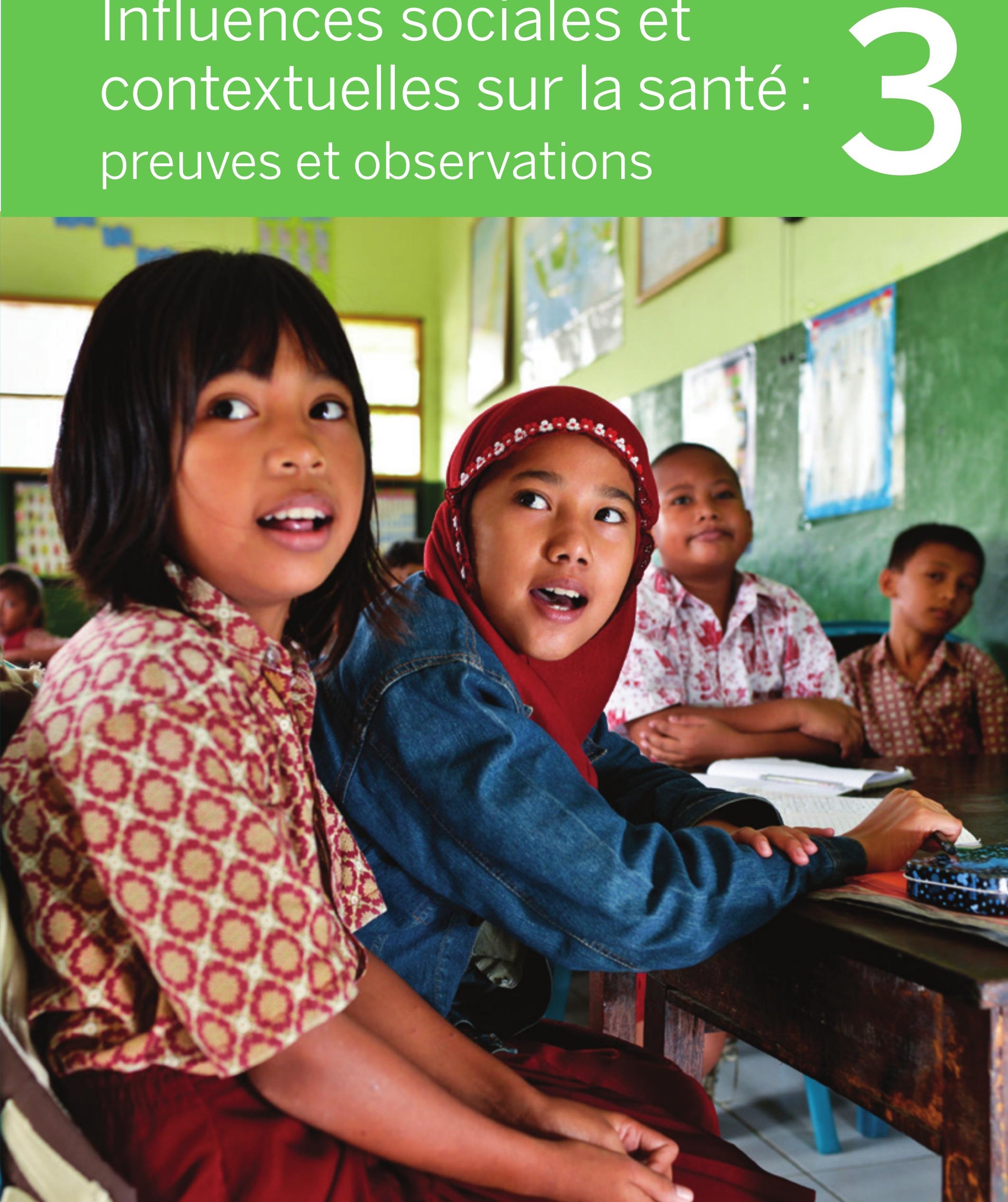


\subsection{Les domaines critiques dans la vie des jeunes adolescentes et adolescents}

Dans cette section, nous examinons de plus près plusieurs domaines sociaux et contextuels clés qui influencent la vie des jeunes adolescents. La multitude de facteurs influençant la santé et le bien-être des TJA couvre les attributs personnels (par ex., l'âge, le genre, le patrimoine génétique); l'environnement immédiat (par ex., les parents et l'école, le statut socio-économique); l'environnement physique (par ex., la prévalence des maladies, la qualité du logement et la pollution, les communautés dénuées de ressources); et les politiques et les lois qui réglementent les conditions où résident les TJA (OMS2014b).

L'âge entre 10 et 14 ans est généralement une période où les filles et les garçons résident avec leurs parents et sont inscrits à l'école. Lorsque c'est le cas, les TJA passent beaucoup de temps dans ces environnements. Ainsi, même si les pairs, les partenaires, les prestataires de services et d'autres adultes importants ont aussi une influence essentielle dans la vie des TJA, nous nous concentrons ici sur deux influences qui sont sans doute les plus importantes pour une grande partie des jeunes adolescents: l'école et les parents.

Le fait de résider avec ses parents et/ou d'être scolarisé permet d'étendre ses atouts sociaux, comme les réseaux sociaux, l'accès aux institutions de la société et les relations de confiance. Le fait de vivre avec ses parents et/ou d'aller à l'école peut également augmenter le capital humain, y compris les connaissances et les compétences, l'estime de soi et l'efficacité personnelle pour adopter des comportements et prendre des décisions. Par exemple, environ $50 \%$ des adolescents interrogés pendant une étude de l'OMS ont mentionné que leur famille était une source d'informations sur la santé (OMS 2014b).

En revanche, les TJA qui ne sont pas scolarisés et/ ou qui ne résident pas avec l'un de leurs parents ou les deux peuvent manquer ou avoir un accès réduit aux atouts offerts par ces systèmes de soutien et, par conséquent, courir un risque plus élevé de connaître divers effets négatifs. Il est égale- ment important de noter que les autres influences pouvant avoir lieu au sein ou en dehors de l'école et des environnements familiaux peuvent diminuer l'utilité de ces domaines. Par exemple, étant donné les changements sociaux et liés au genre qui ont lieu au début de l'adolescence, les normes de genre inculquées au sein des environnements immédiats de l'école, la famille ou d'autres espaces peuvent être à l'origine des disparités dans la capacité des TJA à accéder à et acquérir des atouts (Igras et al. 2014; OMS 2014b).

L'exposition à la violence ou le fait d'être victime dans ces contextes pourraient fragiliser davantage la santé et le bien-être. Une étude internationale sur les perceptions des adolescents concernant la santé dans leur communauté a révélé que le genre et la sécurité personnelle étaient les préoccupations majeures des jeunes en termes d'obstacles à une bonne santé (Mmari et al. 2014). Enfin, les sous-ensembles de TJA ayant des vulnérabilités particulières peuvent être davantage à risque car leur capacité d'accès aux systèmes de soutien, comme l'école et la famille, peut être interrompue dans des situations d'urgence et de crises humanitaires, de migration ou de trafic d'êtres humains.

Nous reconnaissons que la pauvreté est à la fois une cause et une conséquence de la mauvaise santé et que la vulnérabilité économique influence de manière radicale les trajectoires de vie des filles et des garçons de manières différentes. Bien que ce sujet soit d'une importance capitale, ce rapport n'est pas en mesure d'aborder l'influence de la pauvreté de manière aussi complète que ce sujet le mérite. Nous notons que l'interaction des conditions de pauvreté sur le bien-être des adolescents fait l'objet d'autres études en cours (Mmari et al. 2014; Decker et al. 2014).

Nous commençons cette section en présentant une vue d'ensemble des indicateurs de ces dimensions disponibles dans les enquêtes démographiques et de santé (EDS) à l'échelle nationale (Tableau3). Nous développons ensuite les principaux facteurs d'influence identifiés pour la santé des TJA (par ex., l'école, les parents, les normes de genre, l'exposition à la violence et les contextes de vulnérabilité particuliers). 


\begin{tabular}{|c|c|c|c|c|c|c|c|c|c|c|c|c|}
\hline \multirow[b]{3}{*}{ PAYS } & \multirow{3}{*}{$\begin{array}{l}\text { ANNÉE DE } \\
\text { L'ENQUÊTE } \\
\text { EDS LA } \\
\text { PLUS } \\
\text { RÉCENTE }\end{array}$} & \multirow{3}{*}{$\begin{array}{c}\% \text { DE LA } \\
\text { POPU- } \\
\text { LATION } \\
\text { ÂGÉE } \\
\text { ENTRE } \\
10 \text { ET } \\
14 \text { ANS }\end{array}$} & \multicolumn{10}{|c|}{ CHEZ LES ADOLESCENTS AGÉS DE 10 À 14 ANS* } \\
\hline & & & \multirow{2}{*}{$\begin{array}{c}\% \\
\text { VIVANT } \\
\text { DANS } \\
\text { LES } \\
\text { ZONES } \\
\text { RU- } \\
\text { RALES }\end{array}$} & \multirow{2}{*}{$\begin{array}{l}\text { \% POUR } \\
\text { LESQUELS } \\
\text { UN PARENT } \\
\text { OULES } \\
\text { DEUX SONT } \\
\text { DÉCÉDÉS }\end{array}$} & \multicolumn{2}{|c|}{$\begin{array}{l}\% \text { NE VIVANT } \\
\text { AVEC AUCUN } \\
\text { DES PARENTS }\end{array}$} & \multicolumn{2}{|c|}{$\begin{array}{l}\text { \% NON } \\
\text { SCOLARISÉS }\end{array}$} & \multicolumn{2}{|c|}{$\begin{array}{c}\text { PARMI LES } \\
\text { SCOLARISÉS, \% } \\
\text { DONT LE NIVEAU } \\
\text { SCOLAIRE NE } \\
\text { CORRESPOND } \\
\text { PAS Â LEUR ÂGE }\end{array}$} & \multicolumn{2}{|c|}{$\begin{array}{l}\text { \% NE VIVANT } \\
\text { AVEC AUCUN } \\
\text { DES PARENTS } \\
\text { ET NALLANT } \\
\text { PAS Á L'ÉCOLE }\end{array}$} \\
\hline & & & & & FILLES & GARÇ. & FILLES & GARÇ. & FILLES & GARÇ. & FILLES & GARÇ. \\
\hline \multicolumn{13}{|c|}{ Afrique occidentale et centrale } \\
\hline Bénin & 2006 & 14 & 63 & 11 & 23 & 15 & 37 & 24 & 27 & 32 & 11 & 4 \\
\hline Burkina Faso & 2010 & 14 & 80 & 9 & 16 & 11 & 49 & 45 & 30 & 33 & 9 & 4 \\
\hline Burundi & 2011 & 13 & 92 & 22 & 17 & 14 & 13 & 11 & 54 & 56 & 4 & 4 \\
\hline Cameroun & 2011 & 13 & 55 & 16 & 27 & 24 & 17 & 11 & 36 & 43 & 3 & 3 \\
\hline Répub. centrafricaine & 1995 & 13 & 55 & 17 & 28 & 25 & 49 & 27 & 45 & 66 & 17 & 8 \\
\hline Chad & 2004 & 14 & 79 & 13 & 17 & 17 & 60 & 44 & 30 & 41 & 9 & 7 \\
\hline Congo & 2006 & 13 & 49 & 15 & 29 & 25 & 8 & 8 & 57 & 62 & 3 & 3 \\
\hline Cote d'Ivoire & 2012 & 13 & 55 & 13 & 34 & 28 & 38 & 25 & 31 & 37 & 16 & 7 \\
\hline Répub. dém. du Congo & 2013 & 14 & 66 & 14 & 23 & 20 & 11 & 7 & 55 & 53 & 4 & 2 \\
\hline Gabon & 2012 & 12 & 15 & 10 & 28 & 25 & 2 & 2 & 46 & 52 & 1 & 1 \\
\hline Ghana & 2008 & 13 & 58 & 11 & 27 & 21 & 12 & 12 & 49 & 52 & 4 & 2 \\
\hline Guinée & 2012 & 15 & 68 & 14 & 26 & 23 & 46 & 34 & 25 & 31 & 13 & 9 \\
\hline Liberia & 2013 & 13 & 41 & 11 & 38 & 29 & 13 & 15 & 78 & 78 & 5 & 5 \\
\hline Mali & 2013 & 15 & 78 & 7 & 16 & 11 & 50 & 44 & 10 & 11 & 10 & 5 \\
\hline Mauritanie & 2001 & 15 & 60 & 12 & 19 & 18 & 34 & 25 & 36 & 34 & 8 & 5 \\
\hline Niger & 2012 & 15 & 83 & 9 & 14 & 9 & 57 & 50 & 9 & 12 & 8 & 5 \\
\hline Nigeria & 2013 & 12 & 60 & 9 & 15 & 12 & 28 & 23 & 30 & 33 & 3 & 2 \\
\hline Sao Tome-et-Principe & 2009 & 14 & 47 & 6 & 27 & 20 & 7 & 7 & 37 & 36 & 3 & 1 \\
\hline Sénégal & 2011 & 13 & 59 & 11 & 23 & 18 & 36 & 37 & 21 & 19 & 9 & 6 \\
\hline Sierra Leone & 2013 & 13 & 68 & 14 & 34 & 30 & 20 & 23 & 43 & 40 & 7 & 9 \\
\hline \multicolumn{13}{|c|}{ Afrique orientale et australe } \\
\hline Afrique du Sud & 1998 & 14 & 54 & 14 & 34 & 33 & 2 & 3 & 11 & 18 & 1 & 2 \\
\hline Comores & 2012 & 13 & 68 & 7 & 25 & 20 & 10 & 10 & 57 & 41 & 3 & 2 \\
\hline Éthiopie & 2011 & 14 & 84 & 14 & 17 & 14 & 24 & 28 & 45 & 44 & 5 & 5 \\
\hline Kenya & 2009 & 14 & 88 & n.d. & n.d. & n.d. & 4 & 5 & 47 & 52 & 0 & 0 \\
\hline Lesotho & 2010 & 13 & 80 & 39 & 36 & 33 & 4 & 11 & 45 & 61 & 1 & 5 \\
\hline Madagascar & 2009 & 15 & 88 & 11 & 20 & 19 & 22 & 22 & 53 & 58 & 6 & 6 \\
\hline Malawi & 2010 & 15 & 87 & 21 & 30 & 26 & 7 & 9 & 64 & 68 & 3 & 3 \\
\hline Mozambique & 2011 & 14 & 70 & 20 & 28 & 25 & 21 & 20 & 52 & 55 & 6 & 6 \\
\hline Namibie & 2007 & 13 & 67 & 26 & 45 & 43 & 5 & 7 & 18 & 24 & 2 & 4 \\
\hline Ouganda & 2011 & 15 & 89 & 18 & 17 & 15 & 6 & 5 & 76 & 78 & 2 & 1 \\
\hline Rwanda & 2011 & 13 & 89 & 21 & 19 & 18 & 5 & 6 & 57 & 61 & 2 & 2 \\
\hline Swaziland & 2007 & 15 & 86 & 33 & 39 & 41 & 8 & 9 & 48 & 61 & 4 & 4 \\
\hline Tanzanie & 2010 & 14 & 79 & 15 & 25 & 20 & 16 & 15 & 19 & 28 & 5 & 4 \\
\hline Togo & 1998 & 15 & 70 & 15 & 29 & 23 & 34 & 15 & 54 & 68 & 12 & 4 \\
\hline Zambie & 2007 & 15 & 64 & 25 & 32 & 29 & 10 & 10 & 38 & 41 & 4 & 4 \\
\hline Zimbabwe & 2011 & 14 & 77 & 33 & 37 & 36 & 6 & 8 & 19 & 25 & 3 & 4 \\
\hline
\end{tabular}




\section{TABLE 3 Suite}

CHEZ LES ADOLESCENTS AGÉS DE 10 À 14 ANS*

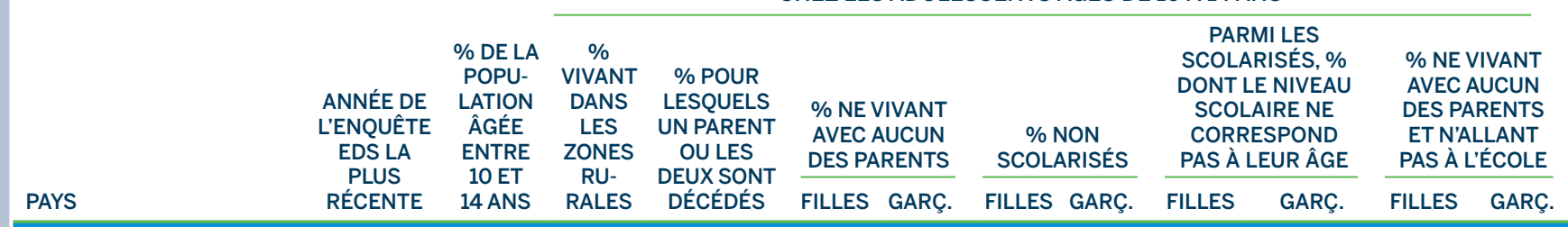

Amérique latine et Caraïbes

$\begin{array}{lcccccccccccc}\text { Bolivie } & 2008 & 13 & 45 & 6 & 10 & 10 & 5 & 3 & 14 & 15 & 1 & 1 \\ \text { Brésil } & 1996 & 12 & 24 & 8 & 12 & 9 & 6 & 6 & 35 & 44 & 2 & 1 \\ \text { Colombie } & 2010 & 10 & 29 & 7 & 13 & 12 & 2 & 4 & 20 & 29 & 1 & 1 \\ \text { Guatemala } & 1999 & 13 & 62 & 9 & 11 & 9 & 25 & 17 & 27 & 34 & 4 & 2 \\ \text { Guyana } & 2009 & 12 & 74 & 9 & 17 & 16 & 4 & 4 & 7 & 7 & 2 & 1 \\ \text { Haïti } & 2012 & 12 & 62 & 16 & 27 & 23 & 5 & 6 & 69 & 75 & 2 & 2 \\ \text { Honduras } & 2012 & 13 & 57 & 9 & 17 & 16 & 13 & 16 & 21 & 29 & 3 & 3 \\ \text { Nicaragua } & 2001 & 14 & 46 & 7 & 13 & 12 & 17 & 24 & 42 & 46 & 3 & 3 \\ \text { Paraguay } & 1990 & 12 & 55 & 0 & 15 & 12 & 17 & 11 & 35 & 45 & 3 & 1 \\ \text { Pérou } & 2008 & 12 & 42 & 6 & 10 & 9 & 4 & 3 & 15 & 18 & 1 & 0 \\ \text { Répub. dominicaine } & 2007 & 12 & 34 & 6 & 22 & 20 & 5 & 7 & 30 & 45 & 2 & 2\end{array}$

\begin{tabular}{|c|c|c|c|c|c|c|c|c|c|c|c|c|}
\hline \multicolumn{13}{|c|}{ Asie du sud et du sud-est } \\
\hline Bangladesh ${ }^{a}$ & 2011 & 12 & 77 & n.d. & n.d. & n.d. & 13 & 18 & 44 & 46 & n.d. & n.d. \\
\hline Cambodge & 2011 & 12 & 86 & 10 & 11 & 11 & 10 & 10 & 42 & 52 & 1 & 2 \\
\hline Inde & 2006 & 12 & 72 & 7 & 6 & 5 & 22 & 16 & 20 & 22 & 2 & 1 \\
\hline Indonésie & 2012 & 10 & 53 & n.d. & n.d. & n.d. & 5 & 5 & 4 & 5 & n.d. & n.d. \\
\hline Maldives ${ }^{a}$ & 2009 & 11 & 75 & 4 & 8 & 7 & 1 & 1 & 7 & 10 & 0 & 0 \\
\hline Népal & 2011 & 13 & 89 & 7 & 10 & 8 & 11 & 5 & 64 & 69 & 1 & 1 \\
\hline Pakistan ${ }^{a}$ & 2012 & 12 & 68 & 7 & 3 & 2 & 36 & 24 & 45 & 56 & 2 & 1 \\
\hline Philippines & 2013 & 11 & 55 & n.d. & n.d. & n.d. & n.d. & n.d. & n.d. & n.d. & n.d. & n.d. \\
\hline Timor-Leste & 2010 & 14 & 78 & 11 & 13 & 12 & 14 & 14 & 44 & 50 & 2 & 2 \\
\hline Vietnam & 2002 & 13 & 86 & n.d. & n.d. & n.d. & 9 & 6 & 10 & 13 & 0 & 0 \\
\hline
\end{tabular}

Moyen-orient et Afrique du nord

$\begin{array}{lccccccccccccc}\text { Égypte }^{a} & 2008 & 10 & 61 & 7 & 1 & 1 & 8 & 7 & 8 & 10 & 0 & 0 \\ \text { Jordanie }^{a} & 2012 & 11 & 18 & 4 & 1 & 1 & 3 & 4 & 1 & 1 & 0 & 0 \\ \text { Maroc } & 2004 & 11 & 47 & \text { n.d. } & \text { n.d. } & \text { n.d. } & 25 & 17 & 25 & 33 & \text { n.d. } & \text { n.d. } \\ \text { Yémen } & 1992 & 15 & 81 & 9 & 0 & 0 & 62 & 8 & 37 & 91 & 0 & 0\end{array}$

Europe et républiques d'Asie centrale

\begin{tabular}{|c|c|c|c|c|c|c|c|c|c|c|c|}
\hline Albanie & 2009 & 10 & 61 & 4 & 1 & 0 & 3 & 2 & 6 & 6 & 0 \\
\hline Arménie & 2005 & 9 & 44 & 4 & 2 & 2 & 2 & 2 & 3 & 2 & 0 \\
\hline Azerbaïdjan & 2006 & 11 & 48 & 4 & 2 & 2 & 4 & 2 & 3 & 4 & 0 \\
\hline Moldavie & 2005 & 9 & 69 & 6 & 14 & 9 & 1 & 3 & n.d. & n.d. & 0 \\
\hline Kazakhstan & 1999 & 12 & 59 & 9 & 5 & 7 & 1 & 1 & 1 & 1 & 0 \\
\hline Ouzbekistan & 1996 & 12 & 65 & 5 & 1 & 1 & 1 & 2 & 1 & 1 & 0 \\
\hline République kirghize & 2012 & 10 & 74 & 6 & 14 & 15 & 1 & 1 & 0 & 1 & 0 \\
\hline Tadjikistan & 2012 & 11 & 77 & 5 & 3 & 3 & 4 & 2 & 1 & 1 & 0 \\
\hline Turquie & 2004 & 10 & 35 & 4 & 3 & 2 & 14 & 5 & 12 & 17 & 0 \\
\hline Ukraine & 2007 & 6 & 39 & 8 & 6 & 6 & 0 & 1 & 2 & 1 & 0 \\
\hline
\end{tabular}

Remarque: Toutes les analyses sont basées sur les données des enquêtes démographiques et de santé. $n$. $d$. . Les données pour les indicateurs n'ont pas été recueillies. * *Les données sur les 10-14 ans sont rapportées par le chef de famille. a Ces pays ont interrogé les femmes ayant déjà été mariées. Les données rétrospectives présentées dans les rapports finaux ont fait l'objet d'un ajustement mathématique pour être représentatives de toutes les femmes. On suppose que les femmes qui n'ont jamais été mariées n'ont jamais donné naissance. 


\section{Élargir l'optique et cibler davantage la vie des jeunes adolescents : utiliser les données d'EDS pour examiner les influences dans l'environnement immédiat des TJA}

Pour fournir un aperçu des contextes dans lesquels les TJA des PRFM résident, nous avons effectué de nouvelles analyses des dimensions de l'environnement immédiat des TJA (statut scolaire, survie des parents et résidence du ménage) tirées des Enquêtes démographiques et de santé (EDS) les plus récentes3 (des années 2011 à 2013). Notre nouvelle analyse fournit des informations portant sur 71 PRFM4 et présente également des données sur les résultats qui reflètent les pratiques culturelles et les normes de genre qui apparaissent pendant la période correspondant à l'âge de 10-14 ans, comme les expériences de mariage, d'initiation sexuelle et de grossesse précoces. Les résultats pour les groupes d'âge 10-14 ans et 15-19 ans sont présentés dans le Tableau 3. Pour une description plus détaillée des informations disponibles dans l'EDS et d'autres programmes d'enquête incluant les adolescents, veuillez vous référer à la Section 5.1.

\subsection{Différences liées au genre dans la transition de l'école primaire à l'école secondaire}

L'éducation est associée à de meilleurs résultats en matière de santé. Comme le montrent les indicateurs sélectionnés concernant l'éducation des TJA, certains progrès ont été accomplis. Comme l'atteste notre analyse EDS présentée dans le Tableau 3, dans la majorité des pays, moins de $20 \%$ des TJA ne sont pas scolarisés. II s'agit d'une amélioration considérable depuis notre rapport de2006, qui montrait que $50 \%$ ou plus des TJA n'étaient pas scolarisés dans de nombreux pays (Chong, Hallman et Brady 2006). Les changements de politiques en vue d'universaliser l'accès

3 Pour plus d'informations, veuillez consulter: www. dhsprogram.com.

4 L'édition2006 de ce rapport incluait l'analyse de données pour 39 pays. à l'éducation primaire ont permis la scolarisation d'un nombre beaucoup plus important d'enfants. Malgré ces améliorations, les TJA non scolarisés sont toujours trop nombreux. Le Tableau 3 montre que dans plusieurs pays d'Afrique occidentale et centrale, et dans quelques pays de la région Moyen-Orient/Afrique du Nord (MOAN), environ la moitié des filles et un tiers des garçons âgés de 10 à 14 ans ne vont pas à l'école.

Notre analyse EDS indique que la transition de l'école primaire à l'école secondaire est susceptible de présenter un défi dans les PRFM, en particulier pour les filles. Même s'il varie en fonction des structures d'éducation nationale, le passage au secondaire se produit souvent après six années complètes d'étude, ce qui même après une entrée tardive dans le primaire et/ou un redoublement, coïncide avec la période de vie des TJA.

Dans le Tableau 3, la variable du « niveau scolaire correspondant à l'âge », fournit des informations indiquant si les adolescents sont en retard par rapport au niveau d'étude qu'ils devraient suivre. Les adolescents qui sont en retard de deux ans ou plus sur leurs pairs dans leur classe actuelle peuvent courir un risque accru d'abandon scolaire (Grant et Hallman 2008; Jimerson, Anderson et Whipple 2002). Nos résultats révèlent que dans une multitude de pays, près de la moitié des 10-14 ans toujours scolarisés n'étudient pas dans une classe correspondant à leur âge. La progression scolaire des garçons est également en retard par rapport à celle des filles.

Une analyse plus approfondie montre que même si les garçons sont plus susceptibles d'entrer à l'école en premier lieu, la probabilité que les filles abandonnent l'école au moment de la puberté semble être supérieure à celle des garçons dans de nombreux contextes (UNESCO 2015). Comme le montrent les études de cas des pays de la Figure 5, la scolarisation est plus faible pour les filles au début de la puberté que pour les garçons.

Une analyse de l'UNESCO (2011) de 14 pays ayant le nombre le plus élevé de jeunes adolescentes non scolarisées démontre que parmi les enfants inscrits en primaire, dans huit pays, les filles ont moins de chance que les garçons de passer au secondaire (Burkina Faso, Burundi, Guinée, Mali, 
FIGURE 5 Pourcentage d'adolescents sco-

larisés, âgés entre 10 et 14 ans et 15 et 19 ans,

quatre pays sélectionnés, 2004-2009

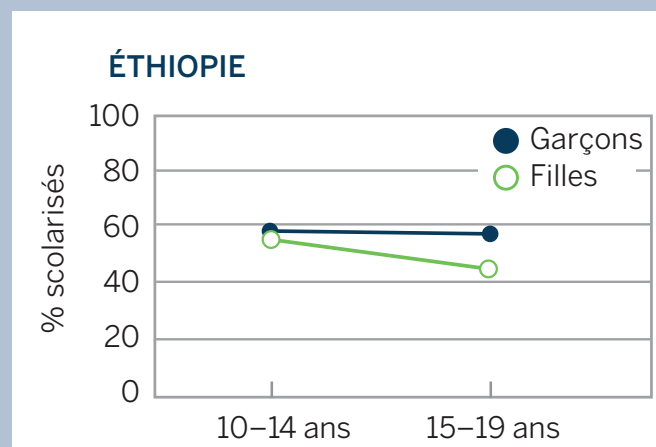

KENYA

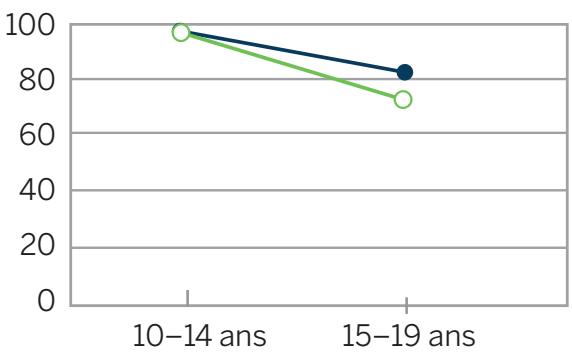

MALAWI

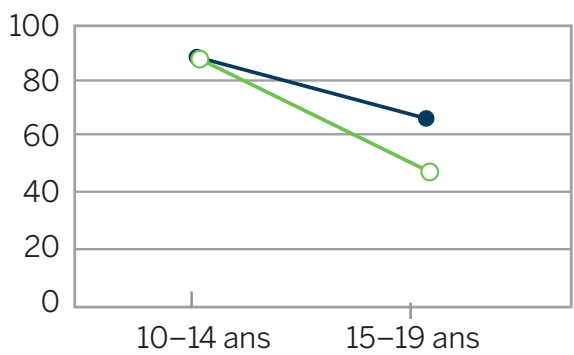

ZAMBIE

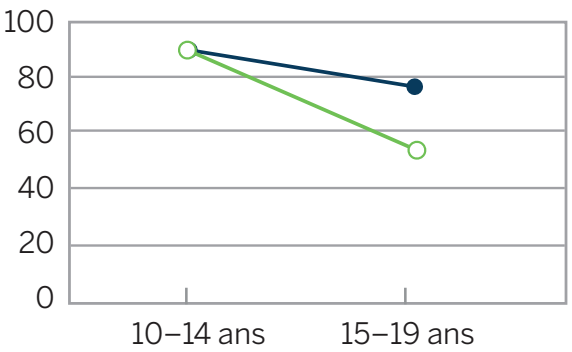

Sources: Enquêtes démographiques et de santé. Éthiopie, 2005; Zambie, 2007; Kenya, 2009; Malawi, 2004. Analyse réalisée par Katharine McCarthy, Population Council, 2013.
Niger, Madagascar, Tanzanie, Ouganda); dans six pays, les chances sont égales (Ghana, Indonésie, Madagascar, Malawi, Myanmar, Pakistan), et dans deux pays (Éthiopie et Mozambique) les filles ont plus de chance de progresser que les garçons (UNESCO 2011). Le fait de ne pas passer au niveau secondaire s'explique par diverses raisons (Banerjee et al. 2013). L'école secondaire implique souvent des frais plus élevés et un temps de trajet et une distance plus importants, ce qui décourage certains parents et tuteurs d'y envoyer les jeunes adolescentes. Malgré des preuves solides indiquant le retour sur investissements offert par des années supplémentaires d'éducation (des revenus de 5 à $15 \%$ supérieurs par année de scolarité atteinte, et des retours supérieurs pour les filles que pour les garçons), les parents et les étudiants sont souvent mal renseignés à propos de ces avantages. Les efforts pour faciliter une transition harmonieuse du primaire au secondaire sont justifiés et abordés dans la Section 4. La violence scolaire est un problème répandu qui peut limiter les avantages de l'éducation (se référer à la Section 3.6).

\subsection{Survie et présence des parents}

Les parents ont la possibilité d'exercer une influence immense sur la santé et le bien-être des TJA. Les jeunes adolescents qui ne résident avec aucun de leurs parents peuvent être particulièrement vulnérables à l'exploitation sexuelle, aux travaux dangereux et à la toxicomanie (Temin et al. 2013). Des données d'EDS portant sur plusieurs pays indiquent qu'une proportion élevée des adolescents de 10 à 14 ans dans certains pays d'Afrique subsaharienne ne vivent avec aucun de leurs parents (Tableau3). Bien que les données rapportées ici ne fournissent pas d'informations détaillées sur leur situation, comme la résidence avec la famille élargie ou un placement dans un foyer ou une famille d'accueil, les résultats soulignent qu'il est nécessaire d'effectuer davantage de recherches.

Dans l'ensemble des régions du monde, davantage de TJA filles que de garçons dans lesPRFM ne vivent avec aucun de leurs parents, tandis que le pourcentage le plus élevé des 10-14 ans chez les 
deux sexes ne vivant avec aucun de leurs parents se situe en Afrique subsaharienne (par exemple, jusqu'à $45 \%$ des filles et $43 \%$ des garçons âgés entre 10 et 14 ans en Namibie) (Tableau 3). Ceci peut être expliqué en partie par une longue tradition de placement des enfants dans cette région. Le pourcentage de TJA dont un ou les deux parents sont décédés est également plus élevé dans les pays d'Afrique subsaharienne, ce qui est probablement dû au fardeau important du SIDA. Comparé à notre analyse de 2006 , le pourcentage de filles et de garçons qui ne vivent avec aucun de leurs parents reste relativement similaire, tandis que l'évolution du pourcentage des 10-14 ans dont un ou les deux parents sont décédés est mitigé et varie en fonction des pays (Chong, Hallman et Brady 2006).

\subsection{Le rôle des normes de genre au début de l'adolescence}

Dans la majeure partie du monde, il a été prouvé que les normes de genre rigides concernant les rôles et comportements appropriés pour les hommes et les garçons et les femmes et les filles influencent de nombreux aspects de la vie des adolescents, y compris les comportements liés à la santé (Barker et al. 2007; Peacock et Barker 2014). Par exemple, le fait que les filles puissent ou non voyager loin de chez elles, les endroits où elles peuvent ou non se rendre dans leur communauté, et la nature et le type d'interactions sociales permises avec le sexe opposé sont fortement influencés par les normes de genre. En général, les garçons ont la possibilité de se déplacer plus librement en dehors de chez eux, ce qui leur offre davantage d'opportunités que les filles de participer à des activités de loisir ou génératrices de revenus (Brady 2005; Barker 2000; Hallman et Roca 2006), et l'avantage masculin concernant l'accès à la communauté et aux espaces à l'intérieur de celle-ci devient plus extrême au moment de la puberté(Hallman et al. 2014).

Les changements positifs des normes sociales, des valeurs et des pratiques liées au genre peuvent avoir un effet transformateur sur la valeur que les sociétés accordent aux filles et la manière dont les TJA perçoivent leurs rôles sociaux et
Dans l'ensemble des régions du monde, davantage de TJA filles que de garçons dans les PRFM ne vivent avec aucun de leurs parents, tandis que le pourcentage le plus élevé des 10-14 ans chez les deux sexes ne vivant avec aucun de leurs parents se situe en Afrique subsaharienne.

sexuels, car les normes de genre jouent un rôle dans leurs perceptions et leurs comportements (Lundgren et al. 2013). Par exemple, un examen systématique à l'échelle mondiale des facteurs influençant les comportements sexuels des jeunes a confirmé que les stéréotypes de genre et les différentes attentes concernant les comportements sexuels appropriés pour les garçons et pour les filles influençaient leur comportement sexuel (Marston et King 2006). Les attentes traditionnelles concernant les rôles liés au genre peuvent entraîner des comportements qui désavantagent les garçons également, notamment le risque de violence, la toxicomanie et les accidents (Barker 2000 et 2007).

Chez les filles, le début de la puberté et le commencement des menstruations ont une profonde signification à la fois au niveau biologique et social, car elles signalent que les filles sont en train de devenir des femmes. Un examen systématique de l'hygiène menstruelle et de la santé chez les jeunes adolescentes dans les PRFM a mis en évidence une honte considérable liée à la menstruation, en raison de son association à la sexualité et à la reproduction (Sumpter et Torondel 2013). Les résultats suggèrent que les filles ont souvent honte de leur incapacité à gérer les règles de manière efficace, et que la menstruation est associée à la saleté et à la pollution. La honte associée aux menstruations peut se traduire par des restrictions et/ou une exclusion des filles et des femmes. Par exemple, dans certains contextes, les filles qui ont leurs règles n'ont parfois pas le droit 
d'effectuer les activités quotidiennes (se rendre à l'église/participer aux activités religieuses, la cuisine et la préparation de la nourriture, établir un contact/toucher un homme, etc.). Bien que certains avancent que la menstruation est un obstacle à la fréquentation scolaire et qu'elle contribue aux taux d'abandon scolaire chez les filles pubères, les preuves sont mitigées (Grant, Lloyd et Mensch 2013).

Nous soulignons brièvement les domaines où les normes de genre émergentes peuvent influencer l'accès des TJA aux ressources.

\section{L'alimentation, la nutrition et le genre}

Le genre a été identifié comme l'un des facteurs influençant les résultats nutritionnels chez les TJA, bien que la direction de cette relation varie en fonction des contextes. Chez les adolescents scolarisés en Afrique subsaharienne, un examen a découvert que les filles étaient plus susceptibles que les garçons d'être en surpoids (Keino et al. 2014). Inversement, dans de nombreux contextes d'insécurité alimentaire, comme en Inde où vit une grande partie de la population sous-alimentée dans le monde, il apparaît que les ménages privilégient la distribution d'aliments riches en nutriments aux garçons mais pas aux filles (More et al. 2013; Keino et al. 2014). Des études menées au Cameroun, en Éthiopie et dans les régions de l'Amazonie ont révélé que les filles vivant dans des ménages en situation d'insécurité alimentaire étaient plus susceptibles de souffrir de retards de croissance que les garçons. Cette différence aurait pour origine les préférences culturelles accordant aux garçons un meilleur accès à la nourriture et à l'éducation (Mukuddem-Petersen et Kruger 2004; Hadley et al. 2008; Piperata et al. 2013). II est possible que de telles pratiques soit le reflet des normes sociales qui accordent davantage de valeur aux garçons qu'aux filles.

Les tabous alimentaires culturels influent également sur l'accès des jeunes adolescents aux aliments et peuvent refléter les normes de genre. Dans certains contextes pauvres en ressources, ces pratiques accordent la priorité aux hommes adultes pour l'obtention de certains aliments riches en protéines, et limitent leur accès aux jeunes adolescents et aux femmes (Meyer-Ro- chow et Benno 2009). Les habitudes et tabous alimentaires sont liés aux transitions clés du cycle de vie, comme la menstruation, la grossesse, l'accouchement et l'allaitement, qui concernent les filles. Bien que les pratiques liées à la grossesse et à l'accouchement puissent être destinées à protéger les filles (ou le développement du fotus), elles peuvent également entraîner un accès restreint aux aliments nutritifs pendant une période où le besoin biologique en micronutriments est particulièrement important (Meyer-Rochow et Benno 2009).

\section{Les technologies de l'information et de la communication : le genre a-t-il un impact sur leur accès?}

Les technologies de l'information et de la communication (TIC), comme la télévision, la radio, les téléphones mobiles et Internet ont le pouvoir d'étendre l'accès des TJA à l'information, leurs liens aux autres et leur capacité à apprendre et à partager des idées. Les TIC sont de plus en plus accessibles dans les PRFM. L'omniprésence croissante des TIC et leur constante évolution font qu'il est essentiel de considérer les avantages et les risques des TIC pour les jeunes. Comme le monde en a été témoin pendant les mouvements de jeunes militants dans l'ensemble du Moyen-Orient en2011 et2012, les jeunes peuvent tirer parti de la technologie en ligne et des médias sociaux pour faire entendre leur voix et avoir une influence puissante sur le changement social et politique (Martin 2012).

Mais l'accès accru des TJA aux TIC présente également des influences potentiellement négatives (Brody 2015), notamment l'exposition aux attentes idéalistes concernant l'image et le corps; la commercialisation d'aliments, de boissons et de comportements sociaux mauvais pour la santé (comme le tabagisme); les moqueries en ligne par les pairs; la diffusion involontaire d'informations privées; et les comportements de prédateurs en ligne. Ces risques mettent également en évidence la nécessité d'équiper les TJA afin qu'ils puissent tirer profit du potentiel offert par les TIC, tout en employant des mesures de protection pour atténuer les risques potentiels (voir la Section 4). 
Tous les TJA n'ont pas un accès égal aux TIC. Chez les 10-14 ans, les données disponibles indiquent que l'utilisation des TIC varie fortement en fonction des pays. Par exemple, parmi les pays ayant des données disponibles en 2011, I'utilisation d'Internet au cours des 12 derniers mois allait de $12 \%$ des 10-14 ans au Paraguay, à 99\% en Slovénie (UNICEF 2012c). De même, I'utilisation des téléphones portables allait de $37 \%$ au Salvador à $98 \%$ en Slovénie (UNICEF 2012c). II existe également des disparités au sein des pays concernant l'accès, plus particulièrement liées au genre. Par exemple, dans les PRFM, les filles et les femmes ont $21 \%$ moins de chances de posséder un téléphone portable que les hommes (GSM Association 2013b).

Si une fille ou une femme réside en Afrique ou au Moyen-Orient, sa probabilité de posséder un téléphone portable est d'un quart inférieure à celle des hommes de son pays, et de plus d'un tiers inférieure si elle réside en Asie du Sud (GSM Association 2013b). Au sein des pays, les personnes qui sont plus éduquées, ont des revenus supérieurs ou résident dans les zones urbaines utilisent généralement davantage Internet que celles qui sont moins éduquées, ont un statut socio-économique inférieur ou vivent dans les zones rurales (UNICEF 2012c).

Les adolescents n'ayant pas accès ou n'ayant pas été formés à l'utilisation des TIC sont moins capables de participer au dialogue mondial pour obtenir des informations, communiquer et se connecter aux autres, et participer aux campagnes sociales. Les téléphones mobiles sont un moyen de participation important, en particulier dans les lieux où l'accès à Internet est limité. Par exemple, les résultats des adolescents âgés de 8 à 18 ans interrogés dans le cadre d'une étude en Afrique du Nord et au Moyen-Orient ont montré que plus de la moitié utilisaient leur téléphone mobile pour accéder à Internet (GSM Association 2013a). Les SMS et les applications de messagerie mobile se sont également développés, représentant de nouvelles manières pour les jeunes d'établir des liens et de partager l'information.

\subsection{La violence à l'égard des TJA: les garçons et les filles sont touchés, mais la prévalence varie en fonction du genre}

L'expérience de la violence a une influence critique sur le bien-être des TJA, y compris sur leur santé physique et mentale et leurs progrès scolaires. La Convention relative aux droits de l'enfant garantit que tous les enfants devraient pouvoir vivre sans subir aucune forme de violence. Pourtant, partout dans le monde les TJA sont soumis aux mauvais traitements infligés par les personnes qui s'occupent d'eux, leurs enseignants, leurs pairs et d'autres personnes. Ces expériences perturbent souvent l'accès des TJA et leur engagement auprès des systèmes de soutien comme la famille et l'école, car la majorité des auteurs de ces violences sont des connaissances de l'enfant et sont souvent précisément les personnes chargées de le protéger.

La première étape pour remédier à ce problème est de reconnaître sa nature et sa prévalence. Malgré de grandes difficultés de mesure, les mauvais traitements à l'encontre des TJA commencent à être quantifiés, grâce à des données de plus en plus nombreuses depuis la fin des années 1990 (voir la Section 5.2. pour les sources de données sur la violence à l'encontre des TJA).

Faire davantage la lumière sur ce problème incite le développement de législations, de politiques et de programmes, dont des campagnes destinées à changer le comportement des adultes pour modifier les normes et attitudes sociales qui contribuent à perpétuer les violences contre les TJA (voir la Section 4). La violence à l'égard des TJA prend plusieurs formes majeures. Les données sur ces formes de violence, recueillies en grande partie par I'UNICEF, sont décrites ci-dessous (UNICEF 2014c).

\section{La violence physique}

Alors que les enfants abordent la deuxième décennie de leur vie, les blessures volontaires et involontaires deviennent toutes deux plus fréquentes (se référer à la discussion sur les années de vie corrigées de l'incapacité chez les TJA dans 
Dans les 58 pays inclus dans la base de données mondiale de I'UNICEF, près de 85 \% des 10-14 ans ont déclaré avoir subi une discipline violente au cours du mois où ils ont été interrogés.

la Section 2.1). Globalement, les blessures sont responsables des décès de $28 \%$ des adolescents âgés de 10 à 14 ans à l'échelle mondiale. Les blessures involontaires comprennent les accidents de la route, les chutes, l'empoisonnement, l'exposition au feu/à la chaleur/aux substances chaudes, l'exposition aux forces de la nature, et autres; celles-ci représentent $25 \%$ des décès chez les 10-14 ans à l'échelle mondiale, contre 15\% chez les 0-9 ans (UNICEF 2014c). La proportion de décès dus aux blessures physiques volontaires (dont les homicides, l'automutilation et la violence collective) chez les garçons et les filles augmente, passant de $0,5 \%$ chez les $0-9$ ans, à $6 \%$ et $5 \%$ respectivement, à l'âge de 10 et 14 ans. En général, dans les PRFM, les taux d'homicides chez les adolescents sont plus élevés dans les pays caractérisés par une répartition inégale des richesses. Chez l'ensemble des 10-14 ans en Afrique occidentale et centrale, et chez les garçons en Amérique latine et dans les Caraïbes, I'homicide représente la part la plus élevée de la charge de morbidité causée par les blessures volontaires. II est alarmant de constater que dans sept pays d'Amérique latine et des Caraïbes, l'homicide est la cause majeure de décès chez les garçons adolescents, et 3 homicides sur 10 dans cette région sont dus à la violence des gangs.

Bien que ces statistiques donnent à réfléchir, beaucoup plus d'enfants subissent la violence sous la forme de discipline par leurs tuteurs et/ou enseignants. Cela se produit généralement chez eux ou dans le cadre scolaire, et commence dès le plus jeune âge. Environ 6 enfants sur 10 dans le monde, âgés de 2 à 14 ans, sont régulièrement soumis à des châtiments physiques (corporels) par les personnes qui s'occupent d'eux. Au sein du groupe d'âge des TJA, dans les 58 pays inclus dans la base de données mondiale de l'UNICEF, près de $85 \%$ des 10-14 ans ont déclaré avoir subi une discipline violente au cours du mois où ils ont été interrogés (UNICEF 2014c).

\section{La violence psychologique}

La violence psychologique à l'encontre des enfants, également appelée violence/abus émotionnel ou mental, est fréquemment commise par des personnes avec lesquelles l'enfant entretient une relation étroite. La plupart du temps, il s'agit des parents et des personnes s'occupant des enfants, mais de nouvelles preuves suggèrent que les enseignants, les pairs et les partenaires amoureux seraient également couramment les auteurs de ces violences (Sedlak et al. 2010; Leen et al. 2013; Theoklitou, Kabitsis et Kabitsi 2012; Shumba 2002). Cette forme de violence fait partie des moins étudiées, et les statistiques font défaut. Ceci est largement dû à la difficulté de conceptualiser et de quantifier les nombreuses formes de cette violence, notamment le fait d'effrayer, de menacer, d'exploiter, de terroriser, de rejeter, d'isoler, d'ignorer, d'insulter, d'humilier et de ridiculiser. Refuser d'offrir un soutien émotionnel, négliger les besoins en matière de santé et d'éducation, exposer un enfant à la violence conjugale et l'intimidation sont également considérés comme une violence émotionnelle. Les formes extrêmes comprennent le fait de soumettre un enfant à l'isolement ou à d'autres formes humiliantes de détention.

Bien que les données de prévalence concernant la violence psychologique à l'encontre des enfants dans les PRFM soient rares, les enquêtes sur la violence contre les enfants (VACS) de I'UNICEF et des Centres pour le contrôle et la prévention des maladies (CDC) ont posé des questions sur cette forme de violence (et d'autres) dans des enquêtes représentatives à l'échelle nationale portant sur les adolescents au Kenya, au Swaziland, en Tanzanie et au Zimbabwe en utilisant des déclarations rétrospectives. Les données disponibles ne sont pas ventilées pour les TJA; cependant, les résultats indiquent que le fait d'avoir déjà connu une violence émotionnelle avant l'âge de 18 ans est légèrement plus courant chez les hommes que chez les femmes. Pour les hommes, la prévalence varie entre 30 et $38 \%$, contre 24 et $30 \%$ chez les femmes (UNICEF 2007; UNICEF, CDC, et Université de Muhimbili 2011; UNICEF et al. 2012; UNICEF 
et Collaborating Centre for Operational Research and Evaluation 2013). Les formes de violence étaient similaires en fonction du sexe et entre les quatre pays. L'humiliation par des membres adultes de la famille et les enseignants était la forme la plus courante, suivie par le manque de soin et les menaces d'abandon de la part des personnes s'occupant des enfants.

\section{La violence sexuelle : un taux plus élevé de déclaration chez les filles, souvent avec des conséquences irrémédiables}

La violence sexuelle est une violation extrême des droits humains des adolescents, et est soumise à une législation internationale spécifique, conçue pour protéger les enfants contre ses formes multiples. La violence sexuelle est souvent liée à d'autres formes de violence. L'abus sexuel est nocif et traumatisant, quelle que soit sa forme. Bien qu'il existe des estimations concernant le nombre d'enfants ayant subi des violences sexuelles, la sous-déclaration représente un défi extrême et entrave les efforts visant à évaluer la véritable ampleur du problème. Les recherches ont démontré que 30 à $80 \%$ des survivants ne révèlent leurs expériences de maltraitance sexuelle pendant l'enfance qu'une fois devenus adultes, et que de nombreux autres gardent le silence tout au long de leur vie (UNICEF 2014c).

Les données sur l'abus sexuel des mineurs varient beaucoup, y compris les définitions, les méthodes d'obtention des données, la conception des études et l'échantillonnage. Les examens systématiques et les méta-analyses des données auto-déclarées tirées des enquêtes reflètent une grande disparité en matière de prévalence.
Des questions rétrospectives sont disponibles dans l'EDS pour 21 pays sur le pourcentage de filles adolescentes (âgées de 15 à19 ans) ayant déjà subi des violences sexuelles et l'âge auquel elles les ont subies pour la première fois. Dans 16 des 21 pays, au moins 1 adolescente sur 5 ayant déclaré avoir été victime de violences sexuelles a indiqué les avoir subies pour la première fois entre l'âge de10 et 14 ans. Des données d'EDS comparables sur l'âge auquel les garçons adolescents (âgés de 15 à 19 ans) ont connu pour la première fois des violences sexuelles n'étaient disponibles que pour quatre pays et le nombre de garçons ayant déclaré de telles expériences n'était pas suffisant pour produire des estimations fiables.

Pour les TJA vivant dans les PRFM, les enquêtes sur la violence contre les enfants (VACS) sont la source de données les plus récentes et les plus détaillées, et incluent souvent les filles et les garçons. Puisque ces enquêtes portent sur une grande variété d'expériences de violence sexuelle, elles génèrent des taux de prévalence globale plus élevés que les questions moins détaillées (Pereda et al. 2009a et 2009b); CollinVézina, Daigneault et Hébert 2013; Andrews et al. 2004; Stoltenborgh et al. 2011; Pereda et al. 2009a et 2009b). De plus, les enquêtes comme les EDS qui limitent les questions aux formes les plus extrêmes de violence sexuelle, comme les rapports sexuels forcés, produiront des taux de prévalence plus faibles car ces actes ne sont pas aussi courants, et lorsqu'ils se produisent, ils sont moins susceptibles d'être déclarés que les formes moins graves. Le Tableau 4 présente une comparaison des données de prévalence des VACS et des EDS pour les rapports sexuels

TABLEAU 4 Pourcentage d'adolescents déclarant avoir subi une expérience sexuelle non désirée au cours de l'année passée, en fonction du type d'enquête

\begin{tabular}{|c|c|c|c|c|}
\hline \multirow[b]{2}{*}{ PAYS (ENQUÊTE) } & \multicolumn{2}{|c|}{ FEMMES } & \multicolumn{2}{|c|}{ HOMMES } \\
\hline & VACS & EDS & VACS & EDS \\
\hline Swaziland (VACS 2007a) & 33 & n.d. & N'ont pas été interrogés & n.d. \\
\hline Tanzanie (VACS 2009ª , EDS 2010c) & 14 & 5 & 6 & n.d. \\
\hline Kenya (VACS 2010 ${ }^{b}$, EDS 2008-09c) & 11 & 4 & 4 & n.d. \\
\hline Zimbabwe (VACS 2011 ${ }^{\mathrm{b}}$, EDS 2010-11c) & 9 & 7 & 2 & n.d. \\
\hline
\end{tabular}

a $13-24$ ans $\quad{ }^{b} 13-17$ ans $\quad{ }^{c} 15-19$ ans. 
non désirés au cours de l'année passée pour les adolescentes et les adolescents. Bien que l'année de l'enquête, la méthode d'échantillonnage et le groupe d'âge varient, les niveaux des VACS pour les adolescentes sont considérablement supérieurs, et ceux des garçons âgés de 13 à 17 ans ne sont pas négligeables.

Bien que l'exploitation sexuelle des enfants (définie comme des rapports sexuels en échange d'argent, de nourriture ou d'autres faveurs) soit probablement une forme d'abus grave et répandue, elle n'est pas rapportée ici en raison du manque de disponibilité des statistiques nationales. Cela est dû au fait que de tels actes, par leur nature même, sont cachés, ce qui fait qu'il est très difficile de déterminer l'ampleur réelle du problème. De plus, parmi les quelques enquêtes explorant ce problème, certaines s'interrogent uniquement sur les actes menés à terme tandis que d'autres incluent les tentatives dans le chiffre global, ce qui rend la comparaison difficile.

\subsection{La scolarité et la violence}

Même s'il n'existe pas de données systématiques sur sa prévalence, il a été établi que la violence scolaire est un phénomène répandu à la fois dans les PRFM et les pays à haut revenu (PHR) (ONU 2015; Leach, Dunne et Salvi 2014). En plus de ses effets psychologiques négatifs et de son incidence sur la santé, son impact sur le niveau de scolarité représente une préoccupation centrale. La plupart des études existantes sur ce problème ont été descriptives ou qualitatives, et les études quantitatives sont principalement de nature transversale (Psaki, Mensch et Soler-Hampejsek 2015). De plus, peu d'études ventilent les données en fonction des catégories d'âge particulières auxTJA, et les preuves les plus nombreuses viennent des pays à haut revenu (PHR).

Il a été démontré que les expériences de violence à l'école dans les PRFM sont liées à l'absentéisme et à l'abandon scolaire (Pereznieto et al. 2010). Par exemple, une enquête représentative à l'échelle nationale des étudiants d'école primaire en Éthiopie a révélé que l'exposition à la violence scolaire réduisait la participation des filles en classe, réduisait leurs résultats scolaires et augmentait les taux de redoublement et d'abandon (Save the Children, Ministère éthiopien de l'Education, et Ministère de la Condition féminine de l'Éthiopie 2008).

Les données d'une recherche plus vaste (UNICEF 2014c) indiquent que le fait de subir tout type de violence pendant l'enfance, que ce soit à l'école ou en dehors, a de graves conséquences sur le succès scolaire (NCAC 2013). Peu de statistiques sont disponibles pour les enfants des PRFM. Mais une étude d'Afrique du Sud (Hallman 2007) a montré que l'expérience déclarée de rapports sexuels forcés, quel que soit le lieu ou l'auteur, était associée à des niveaux plus faibles de scolarisation chez les filles et les garçons, et à une réussite et une progression scolaires inférieures chez les filles. Les TJA qui subissent des formes de violence multiples courent un risque particulièrement élevé de mauvais résultats scolaires. Ceux qui sont intimidés par leurs pairs à l'école et qui subissent aussi des mauvais traitements en dehors de l'école courent un risque plus important d'avoir de mauvais résultats scolaires (Schwartz et al. 2013). Une étude utilisant des données longitudinales du Malawi examine les effets de la violence scolaire et familiale sur les résultats en matière d'éducation, et révèle que la violence familiale est associée à des résultats scolaires plus faibles pour les filles, tandis que la violence sexuelle dans le contexte scolaire est associée à des résultats scolaires plus faibles pour les garçons (Psaki, Mensch et Soler-Hampejsek 2015).

\subsection{Les programmes répondant aux TJA dans des circonstances graves et particulières}

Les adolescents qui grandissent dans des circonstances difficiles (par ex., mariage d'enfants, travail fondé sur l'exploitation des enfants, migration forcée, conflit armé, et violence au sein du ménage et de la communauté) sont poussés à assumer prématurément des rôles et comportements d'adultes. Les chercheurs étudient toujours les effets à long terme de ces transitions accélérées sur le développement des individus et leur capacité future à assumer des rôles d'adulte. Par exemple, les chercheurs ont commencé à explorer les effets à plus long terme liés à la vie 
dans des ménages affectés par le SIDA, l'exposition à la violence, et un statut socio-économique précaire au début de l'adolescence. Un nombre de preuves croissant a documenté les effets néfastes associés au fait de grandir dans des conditions perturbatrices, par exemple, en consignant les problèmes de santé mentale chez les enfants orphelins du SIDA (Han, Ssewamala et Wang 2013). De nombreux autres facteurs comme le conflit armé et la guerre, le déplacement forcé, le statut en matière d'immigration, et les mauvais traitements ou la négligence pendant l'enfance ou l'adolescence ont également été identifiés comme des facteurs de risque de problèmes de santé mentale (Kieling et al. 2011). Dans cette section, nous décrivons les conditions sociales qui contribuent aux vulnérabilités particulières au sein des groupes marginalisés de TJA.

\section{Les TJA dans les contextes humanitaires, de crise et d'urgence : un risque élevé d'abandon scolaire précoce et de mariage}

Les très jeunes adolescents dans les contextes humanitaires sont confrontés à des défis inhabituels, qui peuvent varier en fonction du sexe. Les situations d'urgence peuvent prendre des formes diverses, notamment des catastrophes naturelles, des conflits humains et des épidémies. Bien que la plupart des personnes, quel que soit leur âge ou leur sexe, soient forcées d'assumer de nouveaux rôles pendant les situations de crise, les adolescentes sont les plus susceptibles d'assumer de nouveaux rôles d'exercice des responsabilités au sein de leur famille. Elles prennent fréquemment la tête du ménage lorsque les parents sont absents ou disparus, sont chargées de veiller sur leurs frères et sœurs plus jeunes, et sont celles qui fournissent un abri, de l'eau et de la nourriture. Les filles peuvent involontairement devenir un moyen de survie pour les familles en situation de crise. Elles peuvent quitter l'école, se priver de repas, s'engager dans des moyens de subsistance risqués, assumer une part disproportionnée des tâches domestiques et/ou se marier tôt pour alléger les responsabilités financières de leur famille.

On rapporte que les parents et les filles ont recours au mariage précoce pour se protéger contre les risques perçus ou réels liés aux moyens de subsistance, parce qu'ils croient qu'ils n'ont pas
Pendant les crises humanitaires, les filles peuvent quitter l'école, se priver de repas, s'engager dans des moyens de subsistance risqués, assumer une part disproportionnée des tâches domestiques et/ou se marier tôt pour alléger les responsabilités financières de leur famille.

d'autre alternative. Les risques liés à la grossesse sont également aggravés pour les filles présentes dans des contextes de conflit en raison de l'exposition accrue aux rapports sexuels forcés et de la disponibilité réduite des services de SSR. Ces circonstances les isolent de leurs amis, de leur école et de leurs réseaux sociaux. Un exemple extrême est l'enlèvement par Boko Haram en2014 de lycéennes au Nigeria, qui auraient ensuite été vendues comme esclaves, forcées à se convertir à I'Islam, à épouser leurs ravisseurs et à faire des attentats suicides (CBS News 2015).

Les très jeunes garçons adolescents sont également confrontés à des dangers résultant des crises, y compris l'exploitation sexuelle et des rôles de combattants forcés. En Afghanistan, par exemple, les garçons sont abusés sexuellement et exploités par des groupes armés et de riches chefs de guerre. La pratique du bacha bazzi (jouer avec les garçons) utilise de jeunes garçons adolescents qui dansent dans des vêtements de femmes et sont violés. Les garçons y sont généralement forcés par les circonstances économiques et en tant que moyen de survie (UNICEF 2014c).

Les enfants soldats sont une autre forme d'exploitation des TJA. On estime à 250000 le nombre d'enfants ayant involontairement servi en tant que soldats en 2015, que l'on définit comme des mineurs de moins de 18 ans, faisant partie d'une force ou d'un groupe armé régulier ou non, en quelque capacité que ce soit (War Child 2015). Cette définition inclut non seulement ceux qui portent des armes, mais également les rôles qui ne sont pas liés au combat. Les filles enfants-soldats peuvent être recrutées dans le but de les 
soumettre à des rapports sexuels forcés et/ou au mariage, en plus de servir dans des rôles de combat, et sont particulièrement vulnérables à la violence sexuelle. Bien qu'il soit difficile de l'établir, on estime que les filles représentent entre $10 \%$ et $30 \%$ des enfants soldats (CSI 2015). La grande majorité des enfants soldats sont recrutés de force par des enlèvements, tandis que d'autres sont contraints par des pressions sociales, culturelles ou économiques. Depuis 2015, la présence d'enfants soldats a été rapportée dans au moins 14 pays dans des régions d'Afrique, du MoyenOrient, d'Asie de l'Est et du Pacifique (CSI 2015). II est apparu que le fait de se trouver dans un environnement d'extrême violence a une incidence sur la santé psychologique et physique, et entraîne notamment des troubles de stress post-traumatique, l'abus de substances, la dépression et le suicide (Schauer et Elbert 2010). L'exposition à la violence et au traumatisme en tant qu'enfant soldat interfère avec le développement normal et fait obstacle à la capacité des jeunes de s'intégrer en tant que membres de la société à part entière.

\section{La migration volontaire : courante chez les adolescents, mais on ne connaît pas sa prévalence pour les TJA}

Les très jeunes adolescents qui migrent indépendamment de leurs parents ou tuteurs, comme des migrants adultes, recherchent généralement de nouvelles opportunités économiques et sociales (Edmonds et Shrestha 2009). Lorsqu'ils sont reconnus par les systèmes juridiques, ces enfants sont souvent appelés « mineurs non accompagnés ». De nombreux migrants adolescents, cependant, ne sont pas reconnus en tant que migrants car ils sont identifiés autrement, en tant qu'employés de maison, enfants des rues ou en tant qu'enfants placés en famille d'accueil. Un grand nombre d'entre eux envoient des paiements à leur famille, concilient le travail et la scolarité et parviennent à économiser de l'argent. II existe peu d'informations pour faciliter la comparaison entre les avantages et les nombreux coûts et risques auxquels sont confrontés les migrants adolescents. Les adolescents migrants indépendants peuvent être considérablement affectés par l'absence de protection et de soutien de la part de leur famille et par les défis présentés par leur nouvel environnement après la migration (Yaqub 2009).

Une étude de 2013 a examiné les preuves concernant les facteurs sociaux et économiques de la migration interne pour les adolescentes des pays en développement, et les liens entre la migration, le risque et l'opportunité (Temin et al. 2013). Le rapport s'est concentré sur les manières dont la migration des zones rurales aux zones urbaines peut, lorsque les ressources et filets de sécurité nécessaires sont mis en place, offrir aux adolescentes de nouvelles opportunités qui ne sont pas disponibles dans leur village ou leur ville. Bien que cela puisse être risqué, les recherches ont découvert que pour la majorité des filles, la migration volontaire peut conduire à l'autonomie, aux opportunités et à la prospérité. Certaines filles migrantes parviennent à concilier le travail et la scolarité, et à élargir leurs opportunités, et la plupart gagne suffisamment pour pouvoir envoyer de l'argent à leur famille. Lorsqu'une fille envoie de l'argent chez elle, la manière dont sa famille la perçoit évolue: cela peut lui donner d'avantage d'influence dans la prise de décisions et, dans certains cas, retarder un mariage précoce.

Cependant, pour pouvoir tirer pleinement profit de la migration, les filles ont besoin d'une préparation adéquate avant de migrer, de dispositions pour assurer leur sécurité pendant le voyage et de soutien une fois arrivées à destination. Les programmes et politiques visant à aider les migrants ont largement ignoré les adolescentes, en particulier les très jeunes adolescentes. Dans les rares cas où des politiques et des programmes tentent d'atteindre les migrantes vulnérables, ceux-ci fonctionnent à petite échelle et sont plus susceptibles de bénéficier aux filles migrantes plus avantagées ou aux migrants. De nombreux programmes et gouvernements locaux perçoivent la migration urbaine en des termes négatifs, sans reconnaître ses avantages potentiels (Temin et al. 2013).

Les besoins en matière de SSR des jeunes adolescents qui migrent peuvent être compromis par un manque de liens sociaux à leur destination. La plupart des spécialistes s'accordent 
sur le fait que les migrants ont souvent une bonne santé, et certains aspects du processus de migration peuvent s'avérer protecteurs. Cependant, plusieurs études ont révélé que les migrants adolescents, en particulier les migrants pauvres dans de très grandes villes des PRFM, présentent de mauvais résultats en matière de SSR (Mmari et Astone 2014). Des preuves distinctes portant sur le segment des TJA au sein de la population migrante adolescente font défaut d'enfants.

\section{La traite des personnes: les filles de moins de 18 ans pourraient représenter près de la moitié des victimes de la traite}

La migration volontaire qui, dans l'idéal, implique une planification, une protection et un soutien social, est très différente de la traite des personnes, qui est caractérisée par la tromperie, l'abus, l'isolement social et de multiples violations des droits humains. Bien que la traite des personnes semble être en hausse, sa nature secrète fait qu'il est difficile d'obtenir des estimations précises de sa fréquence. Les nombres rapportés dans les publications sont contradictoires et sont probablement sous-estimés. Une étude de 2013 estime qu'environ 800000 personnes victimes de la traite franchissent des frontières internationales chaque année, et parmi cellesci, $80 \%$ sont des femmes ou des filles et $50 \%$ sont mineures (Deshpande et Nour 2013), ce qui signifie que $40 \%$ de l'ensemble des personnes victimes de la traite sont probablement des filles âgées de moins de 18 ans. Les mineurs n'ont pas été ventilés par segment d'âge dans l'étude.

Les victimes de la traite sont contraintes à différents types de travaux, l'exploitation sexuelle commerciale étant la catégorie principale, suivie par le mariage des enfants (Buckley et Barry 2015; CBS News 2015). Les jeunes femmes et les adolescents sont un groupe particulièrement vulnérable pour le trafic sexuel national et international (Logan, Walker et Hunt 2009). Le commerce mondial du sexe semble être la forme de commerce qui connaît la croissance la plus rapide, avec une valeur annuelle de 32 milliards USD (Deshpande et Nour 2013). Cette violation criminelle des droits humains a de pro- fondes conséquences sur la santé des victimes. Un élément particulièrement préoccupant est l'accès aisé aux très jeunes adolescentes qui sont très recherchées par les «clients» par le biais de sites populaires et légitimes de ventes de services basés sur le web et de sites de réseaux sociaux (McClain et Garrity 2011).

Des recherches provenant des États-Unis indiquent que la pauvreté, l'origine indigène, l'isolement social, la toxicomanie, la violence au sein de la famille, les échecs scolaires, une histoire d'abus sexuels pendant l'enfance, les dysfonctionnements familiaux, et une histoire d'implication dans des activités criminelles rendent les adolescents vulnérables sur le plan émotionnel et économique à la traite (Konstantopoulos et al. 2013; Logan, Walker et Hunt 2009; Williamson et Prior 2009; Small and Justice Policy Center 2008). Pour les adolescentes victimes de la traite internationale, la promesse d'une vie meilleure dans un autre pays est assez fréquente pour leurrer les jeunes filles ou pour que leur famille les place dans les mains des trafiquants (Hodge et Lietz 2007). Ces adolescentes et leurs familles se voient promettre des opportunités légitimes comme le mariage, une carrière de mannequin ou un emploi, mais une fois arrivées dans le pays, sont forcées à servir d'esclaves sexuelles (McClain et Garrity 2011). Les jeunes adolescentes sont très sensibles aux stratagèmes des trafiquants, et l'âge moyen des filles forcées au trafic sexuel est estimé à 13 ans (Shared Hope International 2009). Les victimes du trafic sexuel acquièrent différents problèmes physiques et psychologiques et des désavantages sociaux. Les victimes peuvent être confrontées à des obstacles juridiques, car les trafiquants confisquent ou isolent toutes les formes de documentation concernant leur citoyenneté ou l'immigration. Les obstacles linguistiques et culturels, la peur, les connaissances limitées et le manque d'argent sont d'autres obstacles auxquels sont confrontées les filles victimes de la traite internationale, et qui les empêchent de s'échapper des réseaux de trafic sexuel (Deshpande et Nour 2013). 



\subsection{Identifier les principaux domaines pour les pro- grammes destinés aux TJA}

Dans cette section, nous décrivons les grands domaines programmatiques qui travaillent, ou pourraient travailler, avec les jeunes adolescentes et adolescents dans un éventail de sujets. Dans la mesure du possible (et lorsque les données sont disponibles), nous évaluons les preuves résultant de ces efforts. Comme mentionné précédemment, il existe une diversité immense au sein de la population des TJA (filles/garçons, scolarisés/non scolarisés, mariés/non mariés, ruraux/urbains, etc.), qui a des implications importantes pour l'élaboration de programmes. Nous affirmons que pour être efficaces, tous les programmes doivent, dans un premier temps, identifier clairement le genre et le segment d'âge de la population des TJA ciblée, et doivent être conçus à son intention.

Un principe clé pour une «conception intelligente» est de connaitre les besoins et les intérêts de l' «utilisateur final », dans ce cas précis, la population spécifique des TJA. Un autre principe clé est de travailler à des niveaux multiples de l'environnement des TJA. Appliquer un cadre écologique reconnaissant les relations dynamiques entre l'individu (TJA fille ou garçon), la famille, la communauté et les institutions sociales plus larges peut être extrêmement utile pour penser et concevoir des programmes examinant le sujet dans son ensemble (Figure 6) (Blum et al. 2012). Le cadre écologique examine les effets multiples et l'interdépendance des facteurs sociaux sur les personnes, et est développé à partir des facteurs de niveau immédiat, notamment les influences parentales, scolaires, les normes de genre et la violence abordées dans la Section 3. La mise en œuvre de politiques et programmes adaptés au genre et examinant le sujet dans son ensemble, qui reconnaissent et répondent aux besoins uniques de cette phase de la vie permet de faciliter une transition saine vers l'âge adulte (Blum, Astone et Decker 2013).

Du point de vue programmatique, les points de contact potentiels pour les jeunes enfants comprennent les programmes de vaccination et de santé infantile, ainsi que les efforts d'inscription scolaire, tandis que les adolescents plus âgés peuvent être ciblés dans le cadre d'une éducation par les pairs ou d'autres activités. Les TJA passent souvent entre les mailles du filet, car ils sont trop âgés pour la plupart des interventions de santé infantile. Les filles en particulier sont trop jeunes pour participer aux programmes de santé maternelle et d'autonomisation des femmes. Dans la plupart des pays, il existe des politiques exigeant la scolarisation des enfants de cet âge, et nous constatons une proportion plus importante de TJA scolarisés qu'il y a 10 ans (Chong, Hallman et Brady 2006) et par rapport aux adolescents plus âgés. En même temps, beaucoup de TJA ne sont pas scolarisés et peuvent donc être exposés à un risque plus élevé d'effets négatifs. II est nécessaire d'avoir une compréhension plus nuancée de la variété et du type de circonstances auxquelles sont confrontés les adolescents vulnérables pour créer des programmes efficaces et adaptés aux besoins.

L'intensification ou le «passage à l'échelle » des programmes est un sujet fréquent de discussion, mais qui a fait l'objet de peu d'études rigoureuses dans le domaine des programmes pour les adolescents (Brady 2011b). Bien qu'il soit nécessaire de mettre en œuvre les interventions efficaces à grande échelle, les défis sont considérables. Nous savons, par exemple, que pour développer un projet pilote à grande échelle, il est nécessaire d'accorder de l'attention à l'orientation du contenu, à la structure et au coût des programmes. Nous savons que rester fidèle à l'intervention d'origine est également un défi. À moins d'avoir été soigneusement planifiés dès le début, les projets de démonstration pilote sont souvent intensifs et coûteux, ce qui réduit la probabilité d'une adoption à grande échelle. Souvent, la capacité institutionnelle et de gestion nécessaire pour le développement d'un programme n'est pas en place, et les budgets des pays disposent rarement des fonds nécessaires.

Bien qu'il existe un nombre croissant de recherches sur le développement des interventions de santé (Simmons, Faijans et Ghiron 2007; ExpandNet n.d.; Kohl 2011), il existe moins de connaissances et de recherches concernant l'ex- 


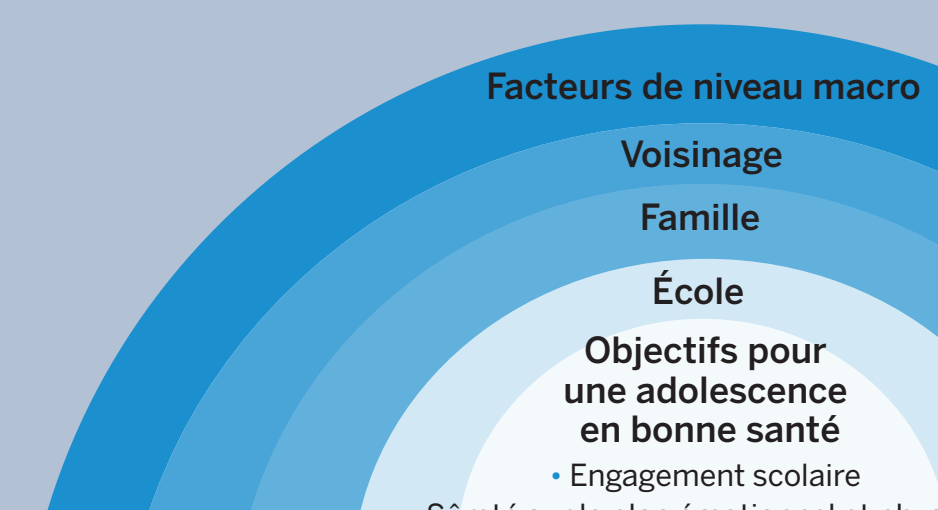

- Sûreté sur le plan émotionnel et physique

- Sens positif de soi ou

de l'efficacité personnelle

- Compétences nécessaires à la vie courante et compétences décisionnelles

Sécurité $\begin{gathered}\text { Bonne santé physique } \\ \text { et mentale }\end{gathered}$

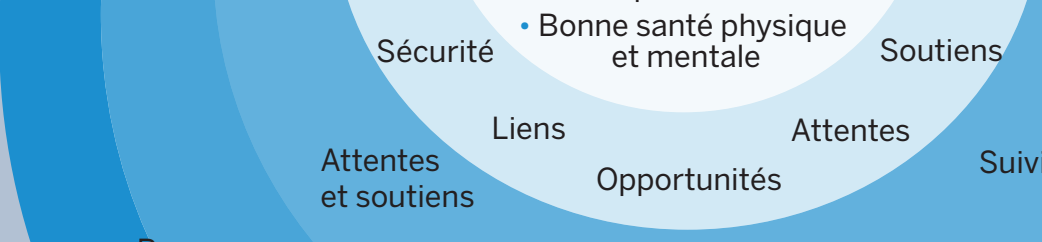

Suivi

Ressources

de la communauté

Liens sociaux

Régulation

comportementale Risques

Normes

de genre

Événements naturels
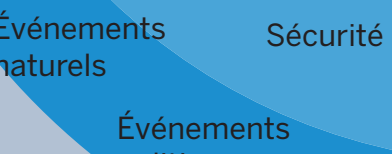

politiques

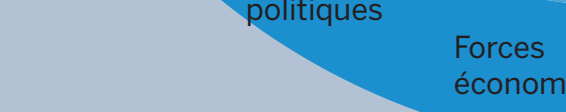

économiques

communautaires

Ressources économiques

(

\section{historiques}

Croyances ou

attitudes

Socialisation

culturelles

\section{Priorités} nationales

collective

Cours de la vie

Pré- $>$ Périnatal Nourisson > Enfant Début de

conception 
Il existe relativement peu de programmes pour les TJA à grande échelle qui reposent sur des recherches sérieuses, et le nombre de programmes ayant fait l'objet d'une évaluation rigoureuse est encore plus faible.

pansion des programmes pour les adolescents en général et pour les interventions concernant les filles et les TJA en particulier. Étant donné qu'il s'agit d'un domaine relativement nouveau à un stade précoce d'expérimentation, la base de connaissances concernant les stratégies les plus efficaces est encore émergente. De plus, comme la plupart des programmes pour les adolescentes sont multisectoriels, le fait d'identifier les institutions appropriées pour adopter l'innovation est une étape clé dans le parcours essentiel menant à l'expansion du projet. À partir de maintenant, des recherches sont nécessaires à la fois pour guider le processus d'expansion et pour mesurer l'impact de ces efforts.

Il convient de noter qu'il existe relativement peu de programmes à grande échelle qui reposent sur des recherches sérieuses, et le nombre de programmes ayant fait l'objet d'une évaluation rigoureuse est encore plus faible. De plus, la plupart des programmes n'accordent pas une attention explicite aux besoins et risques distincts des jeunes filles et garçons adolescents. Cela dit, plusieurs grands domaines thématiques méritent davantage de considération et d'expérimentation. Nous soulignons certains d'entre eux dans cette section. Plutôt que de nous concentrer sur les programmes individuels spécifiques, nous tentons de brosser un tableau d'ensemble des domaines d'intervention. Pour des informations plus détaillées sur les programmes et les ressources concernant les TJA, veuillez consulter: www. k4health.org/search/site/VYA\%20alliance.

\subsection{Programmes pour lesquels nous disposons de preuves solides}

\section{L'éducation complète à la sexualité}

Malgré le peu de données disponibles sur les résultats en matière d'activité sexuelle et de santé reproductive pour les jeunes adolescentes et adolescents (comme indiqué dans la Section2.3), il est clair que les très jeunes adolescents, autant les garçons que les filles, qu'ils aient ou non une activité sexuelle, pourraient bénéficier d'une éducation complète à la sexualité (ECS) adaptée à l'âge. Les militants et les chercheurs ont lancé un appel en faveur de l'ECS (UNESCO 2009; UNFPA 2010). En adoptant une approche basée sur l'autonomisation, l'ECS permet aux jeunes de se voir eux-mêmes et les autres comme des membres égaux dans les relations, et comme des personnes capables de participer à la société. II est important de mentionner qu'un examen de 2015 des programmes d'éducation à la sexualité a révélé que très peu d'entre eux prennent en compte le genre et le pouvoir; ceux qui le font, par contre, obtiennent de meilleurs résultats en matière de SSR (Haberland 2015). Suite à cet examen et à cette analyse, il apparaît nécessaire d'accorder davantage d'attention et de ressources à une éducation à la sexualité qui adopte une approche basée sur le genre et sur les droits.

\section{Les programmes visant à transformer les rôles et les normes de genre}

Le début de l'adolescence est un moment opportun pour intervenir avec des programmes progressistes et basés sur l'égalité entre les sexes avant que des normes de genre nocives ne soient enracinées. Les recherches suggèrent qu'il est possible de remettre en question les rôles dominants de la masculinité et de la féminité en les remplaçant par d'autres modèles (Barker et al. 2007; Peacock et Barker 2014). Les programmes visant à faire évoluer les inégalités entre les sexes-ceux qui promeuvent l'égalité des femmes et des hommes et remettent en question les normes et les rôles dominants basés sur l'inégalité entre les filles et les garçons-peuvent fournir la base nécessaire pour améliorer divers résultats, 
y compris la SSR et la non-violence, entre autres (Lundgren et al. 2013).

Plusieurs programmes ont expérimenté cette approche. Par exemple, «Keep It REAL » en Ouganda, le projet GREAT (Gender Roles, Equality, and Transformations) en Ouganda, et le programme ISHRAQ en Haute-Égypte, œuvrent en vue de transformer les normes de genre par le biais de diverses stratégies (Selim et al. 2013; IRH et USAID 2010). Au Népal, le programme CHOICES aide les jeunes adolescents et adolescentes à découvrir d'autres points de vue sur les rôles de genre traditionnels (Lundgren et al. 2013). Les programmes synchronisés axés sur le genre (qui fonctionnent avec les hommes et les femmes, de manière simultanée ou en séquence) apparaissent désormais comme une approche importante pour faire évoluer les normes de genre (IRH et USAID 2010). Bien qu'ils soient prometteurs, ces programmes synchronisés axés sur le genre nécessitent davantage d'expérimentation et d'évaluation.

\section{L'éducation à la puberté}

Le moment de la puberté ou précédant celle-ci est un point d'intervention logique et pertinent. La puberté est un domaine souvent négligé dans les programmes, il existe donc peu de données sur cette période. Comprendre les implications physiologiques, émotionnelles et sociales de la puberté est essentiel pour atténuer les risques nutritionnels et de santé sexuelle et reproductive chez les adolescents (Sommer 2011). La période du début de l'adolescence offre une occasion unique pour les interventions de santé, commençant avec l'éducation à la puberté, l'hygiène, la conscience du corps, etc. Récemment, un certain nombre de projets dont, notamment, le programme «Grow UP Smart» au Rwanda, se sont concentrés sur cette question (IRH 2015a). Un certain nombre de ressources pédagogiques, comme «My Changing Body », qui se concentrent spécifiquement sur la puberté, ont été élaborées et sont utilisées dans un certain nombre de contextes (IRH 2015b).

Le moment de la puberté constitue également un point d'intervention potentiel car il coïncide avec l'engagement dans le système de santé (par ex., les écoles qui exigent un examen de santé physique ou la vaccination). Certaines recherches
Comprendre les implications physiologiques, émotionnelles et sociales de la puberté est essentiel pour atténuer les risques nutritionnels et de santé sexuelle et reproductive chez les adolescents.

ont examiné la faisabilité et l'efficacité d'associer diverses interventions de santé spécifiques pendant cette période. Par exemple, l'Alliance mondiale pour les vaccins et la vaccination a réalisé un examen systématique des interventions de santé pour les adolescents, qui peuvent être mises en place en même temps que l'administration du vaccin contre le papillomavirus humain (VPH) auxTJA, avec une supplémentation en fer, et l'éducation à l'hygiène menstruelle et à la SSR, entre autres (Hindin, Bloem et Ferguson 2015). Bien que les synergies existent, il est nécessaire d'effectuer davantage de recherches.

\section{L'éducation basée sur l'acquisition de compétences nécessaires dans la vie courante}

L'éducation basée sur l'acquisition de compétences nécessaires dans la vie courante (LSE, Life Skills Education) est perçue par beaucoup comme un mécanisme important pour doter les jeunes des compétences et ressources nécessaires pour surmonter les risques et les défis de l'adolescence et être en mesure de participer de manière productive à la société (UNICEF 2012b). L'éducation aux compétences de la vie courante est utilisée pour enseigner un vaste ensemble de compétences sociales et comportementales conçues pour permettre aux jeunes de gérer efficacement les besoins de la vie quotidienne. Elle a pour objectif d'enseigner diverses compétences et connaissances comme: la pensée critique et créative, la prise de décision, une communication efficace, ainsi que des compétences pour développer des relations saines et une image de soi positive. Ces compétences sont considérées 


\section{Les programmes d'épargne bien conçus peuvent donner aux filles l'autonomie nécessaire pour prendre les bonnes décisions dans des domaines essentiels de leur vie, comme les soins de santé et l'éducation.}

comme universellement valables, mais certaines compétences psychosociales sont considérées comme particulièrement pertinentes pour lutter contre des risques spécifiques comme le VIH ou la violence, entre autres.

Bien que les programmes axés sur les compétences nécessaires dans la vie courante et adaptés au genre et à l'âge soient prometteurs, une évaluation plus rigoureuse est nécessaire. Jusqu'à présent, il n'y a eu aucun examen systématique de l'impact de l'éducation aux compétences de la vie courante sur des résultats spécifiques pour les TJA, que ce soient les filles ou les garçons. Étant donné les définitions diverses données à ce type d'éducation, la variété des modèles de mise en œuvre, et la diversité des domaines thématiques (par ex., VIH/SIDA, la santé et l'environnement, etc.), il est particulièrement difficile de mesurer les résultats. Le fait de clarifier quelles compétences de la vie courante sont pertinentes pour les filles et les garçons, à quels âges, dans quels contextes particuliers, et le meilleur modèle de programme pour leur prestation, permettra une mesure plus solide des résultats.

\section{L'éducation financière et les économies}

Les adolescents sont à la fois les acteurs économiques actuels et futurs dont les décisions et les actions façonneront le développement de leur société. La promotion de l'éducation financière pour les jeunes est essentielle pour assurer une population ayant des connaissances financières et capable de prendre des décisions informées. Le fait de disposer des compétences nécessaires pour gérer les ressources financières à un jeune âge peut atténuer la vulnérabilité sociale et financière et réduire ainsi le risque de pauvreté (UNICEF 2012a). L'introduction de l'éducation financière à un stade précoce, alors que les jeunes sont encore en train d'élaborer des comportements personnels dans le domaine financier, comporte des avantages (Mandell 2009). Le début de l'adolescence est le moment de développer des compétences essentielles en matière de gestion d'argent et des habitudes d'épargne. Ces compétences aideront les adolescents à atteindre des objectifs à court terme et à répondre aux besoins financiers quotidiens, à atténuer les effets des situations d'urgence du ménage, et à prévoir un futur plus sain et plus stable (Austrian et Muthengi 2013).

L'éducation financière peut offrir aux jeunes adolescents une meilleure compréhension des questions financières en leur enseignant les principes de la planification, de la gestion d'argent, de la budgétisation, des économies et des dépenses, des investissements et du crédit. De plus, l'éducation financière expose les jeunes à divers types de systèmes financiers et leur enseigne comment interagir de manière efficace avec différents modes de prestation de services, comme la banque en ligne et par téléphone (UNICEF 2012a). Ce type d'éducation, associé à la possibilité de participer à des systèmes d'épargne (que ce soit des économies individuelles ou des clubs d'épargne de groupe), peut s'avérer un outil extrêmement puissant pour les jeunes. Une expérience réalisée en Afrique du Sud a montré que l'éducation financière améliore le capital social des filles (pas celui des garçons), et que le fait d'avoir un objectif financier était associé à une plus grande probabilité que les filles accèdent au dépistage du VIH (Hallman, Govender et Roca 2007 et 2015).

Un examen systématique récent des études de recherche sur l'éducation financière a révélé une grande diversité de programmes et de contenu fournis de diverses manières et dans différents contextes. Les résultats de cet examen suggèrent que l'éducation financière est efficace pour améliorer les connaissances, les attitudes et les comportements, et produit des avantages tangibles en matière de capacité financière chez les enfants et les jeunes (O'Prey et Shepard 2014). 
Bien que le degré de qualité méthodologique varie en fonction des études, certaines des études les plus rigoureuses sont également les plus récentes, ce qui suggère que la base de preuves s'élargit au fil du temps. L'étude YouthSave de 2015 sur quatre pays PRFM a révélé des résultats prometteurs pour les TJA et a indiqué l'importance de faire baisser l'âge limite légal pour les comptes d'épargne, de permettre d'autres cosignataires adultes pour les comptes lorsque les parents sont absents ou décédés, d'améliorer l'accès des filles aux documents d'identité et de réduire les contraintes spatiales des filles en fournissant des services bancaires là où elles se rassemblent déjà (Johnson et al. 2015).

Il existe une reconnaissance croissante de la part des gouvernements nationaux, des organisations multilatérales et non gouvernementales (ONG) et des prestataires de services financiers de l'importance de l'éducation financière pour les adolescents. Cependant, il n'existe pas de meilleures pratiques universellement acceptées pour intégrer l'éducation financière, sociale et axée sur les moyens de subsistance pour les jeunes adolescents. Au cours de ces dernières années, davantage d'attention a été accordée à l'éducation financière et aux programmes d'épargne pour les adolescents, en particulier les filles. II s'agit d'une possibilité importante pour les interventions, étant donné qu'à mesure que les adolescentes grandissent et assument davantage de responsabilités personnelles et familiales, le besoin d'accéder à l'argent augmente également. Les programmes d'épargne bien conçus peuvent donner aux filles l'autonomie nécessaire pour prendre les bonnes décisions dans des domaines essentiels de leur vie, comme les soins de santé et l'éducation. Nous plaidons en faveur d'une attention accrue pour les programmes et les recherches dans ce domaine.

\section{Les efforts visant à réduire le mariage des enfants}

Pendant la dernière décennie, le nombre de programmes de prévention du mariage d'enfants a augmenté; le nombre le plus important de programmes évalués se trouve en Asie du Sud, en particulier au Bangladesh et en Inde, ainsi qu'en Éthiopie. Les programmes dans un ensemble
Un ensemble de stratégies axées sur l'autonomisation des filles, la mobilisation des communautés, l'amélioration de la scolarité, les incitations économiques (y compris les transferts d'espèces), et les changements politiques ont montré leur efficacité pour réduire le mariage d'enfants dans plusieurs contextes.

plus large de pays d'Afrique et du Moyen-Orient contribuent également aux données de base sur les interventions efficaces (Malhotra et al. 2011). Les recherches visant à déterminer les stratégies les plus efficaces pour réduire le mariage d'enfants ont été effectuées ou sont en cours dans un certain nombre de contextes. Par exemple, au Bangladesh et en Éthiopie, le Population Council et ses partenaires mettent en œuvre des programmes à composantes multiples visant à retarder l'âge du mariage (Erulkar et Muthengi 2009; Amin et al. 2014).

Bien qu'aucune approche unique n'ait prouvé son efficacité pour lutter contre le mariage d'enfants dans tous les contextes, un ensemble de stratégies axées sur l'autonomisation des filles, la mobilisation des communautés, l'amélioration de la scolarité, les incitations économiques (y compris les transferts d'espèces), et les changements politiques ont montré leur efficacité pour réduire le mariage d'enfants dans plusieurs contextes (Malhotra et al. 2011; Erulkar et Muthengi 2009; Amin et al. 2014). Une étude réalisée en 2011 par le Centre international de recherche sur les femmes a identifié 150 initiatives pour empêcher le mariage d'enfants qui fournissent des compétences et une éducation pour les filles et encouragent les familles et les communautés à changer les normes de genre qui perpétuent le mariage précoce. Les programmes œuvrant directement en vue d'améliorer l'autonomie des filles et de leur famille semblent être plus efficaces que les efforts visant seulement à changer les lois ou les politiques (Malhotra et al. 2011). 


\section{Le rôle des tuteurs dans la vie des TJA : la promesse des interventions basées sur les compétences parentales}

Les statistiques présentées dans la Section 3.3 montrent que la plupart des TJA résident avec un de leurs parents ou les deux. Les parents et les tuteurs représentent donc une ressource puissante et sous-utilisée pour améliorer les résultats concernant les adolescents dans les PRFM. Les recherches montrent que des compétences parentales plus solides chez les personnes s'occupant de TJA peuvent réduire les impacts nocifs sur le développement des adolescents liés au fait de résider dans un milieu disposant de peu de ressources (Mejia, Calam et Sanders 2014). Bien qu'il existe de grandes variations entre et au sein desPRFM, beaucoup d'entre eux sont caractérisés par des niveaux de pauvreté élevés, les inégal- ités en matière de revenus et la désorganisation sociale, et les systèmes de soutien social et de santé mis en place pour surmonter ces difficultés manquent souvent largement de ressources (Knerr, Gardner et Cluver 2013). Ces conditions augmentent le risque de violence interpersonnelle ou au sein de la famille (IOM 2008) (abordé dans la Section 3.5). La pauvreté elle-même peut exacerber les risques de difficultés émotionnelles et comportementales chez les enfants et dans les familles (Slopen et al. 2010). De telles circonstances soulignent la nécessité d'interventions parentales efficaces dans les PRFM (OMS 2001; OMS 2009).

Les programmes de compétences parentales dans les PRFM ont un potentiel important. Ces interventions présentent une opportunité d'augmenter les pratiques parentales positives, d'améliorer les relations entre parents et enfants

\section{L'ÉDUCATION}

\section{LES OBSERVATIONS CLÉS ISSUES DE LA DOCUMENTATION DANS LES PRFM COMPRENNENT:}

- Le pourcentage des TJA dans les PRFM achevant l'enseignement primaire a fortement augmenté entre 2000 et 2015.

- Les taux d'achèvement des études primaires restent très faibles en Afrique occidentale et centrale et dans certains pays du Moyen-Orient et de l'Afrique du Nord (MOAN).

- Les parents et les étudiants sous-estiment les avantages économiques que peuvent offrir plusieurs années supplémentaires d'éducation, et n'investissent donc pas suffisamment dans la scolarité.

- Les transferts conditionnels en espèces (TCE) et les transferts inconditionnels en espèces (TIE) augmentent la participation des TJA à l'école primaire. Les TCE sont plus efficaces que les TIE pour les deux sexes, mais plus particulièrement pour les filles.

- Les interventions de santé dans le contexte scolaire augmentent la participation à l'école primaire.

\section{LE RÔLE DES PARENTS}

\section{LES OBSERVATIONS CLÉS ISSUES DE LA DOCUMENTATION DANS LES PRFM COMPRENNENT:}

- Peu d'études sont axées sur le groupe d'âge des TJA.

- La plupart des études portent soit sur les résultats pour les parents, soit sur les résultats pour les adolescents, mais peu d'entre elles portent sur les deux.

- Parmi les études qui évaluent les pratiques parentales, la grande majorité évalue uniquement la communication parent-enfant.

- Les autres comportements parentaux, comme l'engagement et les méthodes disciplinaires, ne sont pas suffisamment mesurés.

- Une minorité d'études évaluent les connaissances ou les attitudes parentales.

- Seule une étude a évalué les résultats concernant le comportement des enfants.

- Concernant les méthodes de déclaration, il n'existe aucune étude présentant une observation directe des interactions parent-enfant (considérée comme la référence absolue en matière de recherche sur les comportements parentaux). 
et de réduire les comportements parentaux abusifs. Ces améliorations ont, à leur tour, montré ensuite des effets sains sur l'architecture du cerveau, le fonctionnement psychologique et social, la santé mentale, les comportements à risque futurs et l'espérance de vie des enfants (Larkin, Beckos et Shields 2012). Malgré les preuves de l'efficacité de ces interventions dans diverses strates socio-économiques et groupes ethniques au sein des pays à haut revenu (Kumpfer et al. 2008), il existe peu d'études portant sur les interventions parentales dans les PRFM.

Bien que les appels lancés par les organismes internationaux pour étendre les recherches à l'échelle mondiale sur ces approches (UNODC 2009; ISPCAN 2007; OMS 2009; Knerr, Gardner et Cluver 2013) aient amorcé la conversation, les études au sein des PRFM demeurent rares, et il existe peu d'évaluations rigoureuses sur les interventions parentales dans les PRFM (Eshel et al. 2006; Butchart 2006; Kumpfer et al. 2008). Un examen de 26 articles de revue de haute qualité sur les approches de prévention de la maltraitance des enfants (Mikton et Butchart 2009) a révélé au total298 études bien structurées, qui venaient toutes, sauf deux d'entre elles, des pays à haut revenu. Un examen des interventions réalisées avec les parents pour promouvoir la santé sexuelle de leurs enfants (Wight et Fullerton 2013) a révélé des évaluations solides de 44 programmes, dont quatre seulement venaient desPRFM. Un examen consacré auxPRFM a étudié les programmes d'éducation parentale positive pour empêcher la violence (Knerr, Gardner et Cluver 2013) et n'a trouvé que 12 évaluations avec des essais randomisés contrôlés (ERC). Mejia et des collègues ont examiné les évaluations publiées depuis 1990 des programmes dans les PRFM pour les parents et tuteurs ayant des enfants âgés jusqu'à 12 ans, et ont trouvé 44 études qualitatives et quantitatives (Mejia, Calam et Sanders 2012).

Parmi les interventions axées sur les compétences parentales dans les PRFM présentes dans les diverses études de revue et par une recherche dans les publications effectuée par les auteurs, six programmes évalués ont été trouvés. Ceux-ci incluaient des participants du groupe d'âge 1014 ans, étaient proposés de manière non sélec-
Les recherches menées dans les milieux à faible revenu ont prouvé que des compétences parentales solides peuvent réduire les impacts néfastes sur le développement des adolescents.

tive aux populations potentiellement à risque et étaient de nature préventive (par rapport à des interventions de traitement proposées à des enfants identifiés comme ayant des problèmes spécifiques). L'encadré intitulé «Observations clés issues de la documentation sur les compétences parentales dans les PRFM» résume les conclusions que nous avons tirées de ces six études (Baptiste et al. 2009; Peña et al. 2008; Phetla et al. 2008; Villarruel et al. 2008; Kagitcibasi, Sunar et Bekman 2001; Van Wyk, Eloff et Heyns 1983; Wight et Fullerton 2013).

Malgré la promesse de ces interventions pour les populations des PRFM, il y a un manque évident d'études de haute qualité sur les interventions parentales en général, et en particulier sur les très jeunes adolescents. II est nécessaire de trouver des études de programmes de compétences parentales auprès des TJA dont la taille d'échantilIon est suffisante, qui possèdent une conception rigoureuse (expérimentale ou quasi-expérimentale), l'évaluation des effets sur le comportement des parents et des enfants, et la mesure des comportements, non seulement par l'auto-déclaration mais aussi par des observations directes des interactions parents-enfants.

\section{Engager et retenir les TJA à l'école}

Bien que le pourcentage d'enfants dans lesPRFM achevant l'école primaire ait beaucoup augmenté entre2000 et 2015 (ONU2015), les très jeunes adolescents continuent à être confrontés à des difficultés en matière d'éducation. Ces difficultés demeurent, même si l'éducation est un droit humain fondamental et malgré la présence de lois rendant la scolarité obligatoire dans de nombreux pays. Comme décrit dans la Section 3.2, la transition de l'école primaire à l'école secondaire 
La transition de l'école primaire à l'école secondaire demeure un défi particulier dans les PRFM et est liée, en partie, à la faible demande des parents pour l'éducation des enfants (en particulier des filles).

demeure un défi particulier dans les PRFM et est liée, en partie, à la faible demande des parents pour l'éducation des enfants (en particulier des filles). La mauvaise alimentation et les infections parasitaires courantes peuvent interférer avec l'apprentissage et la réussite scolaire.

L'environnement scolaire peut entraver la fréquentation et les résultats scolaires. Comme cela a été décrit précédemment, la sécurité à l'école est perçue comme un obstacle à l'éducation, en particulier pour les filles, bien que les données confirmant cette hypothèse soient limitées. L'assiduité des enseignants et les méthodes pédagogiques sont également une source de préoccupation, peu comprise dans les PRFM. Des solutions politiques ont été expérimentées pour surmonter certaines de ces difficultés. Par exemple, les interventions fournissant des informations exactes sur les avantages de la scolarisation ont conduit les parents à revoir leurs croyances concernant la valeur de l'éducation (Nguyen 2008; Jensen 2010). Des données montrent également que les femmes jouant un rôle de modèle positif à l'échelle locale peuvent augmenter la réussite scolaire des filles (Banerjee et al. 2013; Beaman et al. 2012).

Un examen récent des études sur les incitations financières pour l'éducation (Baird et al. 2014) conclut que la fréquentation de l'école primaire et la réussite des études primaires sont sensibles au coût, et que les ménages moins riches connaissent les augmentations de scolarité les plus importantes après la réduction des coûts. Les transferts conditionnels en espèces (TCE) et les transferts inconditionnels en espèces (TIE) améliorent tous les deux les chances d'inscription et de participation scolaire des TJA, avec des effets plus importants pour les TCE. Parmi les pro- grammes de transferts conditionnels en espèces, ceux qui possèdent des règles explicites, un suivi du respect des règles et des sanctions en cas de non-respect ont l'impact le plus important.

L'impact des TCE et des TIE semble être similaire pour les garçons, mais les TCE semblent être plus efficaces pour la participation des filles. Ceci peut s'expliquer par le fait que les parents sont plus susceptibles d'envoyer leurs garçons à l'école de toute façon, et que les conditions associées au transfert servent de mécanisme contraignant pour la fréquentation scolaire des filles. Nous n'avons trouvé aucune recherche sur des expériences de transfert monétaire soutenant la transition du primaire au secondaire; nous les recommandons, en particulier pour les filles.

Il existe peu de preuves indiquant que les incitations monétaires améliorent l'apprentissage des étudiants, en partie parce que les transferts monétaires ont tendance à inclure des étudiants mal préparés dans le système. II n'existe également aucune preuve empirique indiquant que les récompenses financières basées sur les résultats des étudiants augmentent la réussite scolaire. Aucune recherche n'a été trouvée sur l'impact de l'augmentation des crédits monétaires (prêts) aux ménages pour l'éducation dans les PRFM.

Certains problèmes de santé des TJA ont été traités dans le contexte scolaire. Au niveau de l'enseignement primaire, les études ont prouvé que le traitement vermifuge, la fortification en fer et la supplémentation en iode dans le contexte scolaire améliorent la participation scolaire (Kremer et Holla 2009). Cependant, il n'existe pas de preuves tirées d'expériences similaires au niveau secondaire dans les PRFM.

Des études qualitatives indiquent l'importance de l'assainissement dans les écoles pour la scolarisation des filles (Birdthistle et al. 2011). Des recherches utilisant des données scolaires annuelles provenant de l'Inde comparent les écoles ayant reçu des latrines au cours d'une initiative nationale de construction de latrines par rapport aux écoles n'en ayant pas reçu (Adukia 2013). La construction de latrines a augmenté l'inscription de tous les étudiants. Plus jeunes, les filles et les garçons ont considérablement bénéficié d'une latrine, que celle-ci soit unisexe ou non. Concernant 
les adolescents plus âgés, cependant, seules les latrines séparées pour les hommes et les femmes ont augmenté l'inscription des filles, et ces effets ont persisté au moins trois ans après l'installation des latrines.

Des initiatives visant à améliorer la sécurité à l'école font l'objet d'essais pilotes. Les interventions axées sur l'ensemble de l'école, conçues pour lutter contre la violence scolaire sont soutenues par de grandes organisations bilatérales en coopération avec les agences de l'ONU (Fulu, Kerr-Wilson et Lang 2014). L'intervention « Good School Toolkit» élaborée par Raising Voices pour réduire la violence physique du personnel scolaire envers les élèves d'école primaire en Ouganda est le premier essai randomisé contrôlé pour réduire la violence scolaire chez les jeunes adolescents (Devries et al. 2015). L'intervention a inclus une formation à certaines techniques de modification des comportements pour le personnel, les étudiants et l'administration, notamment: fixer des objectifs à l'échelle de l'école, développer des plans d'action avec des dates spécifiques pour les livrables, encourager l'empathie en facilitant la réflexion sur les expériences de violence, fournir des connaissances sur les méthodes de discipline non violentes, et offrir des possibilités de pratiquer les nouvelles compétences comportementales. Les résultats ont également montré que l'intervention a amélioré le sentiment de sécurité des étudiants à l'école, ce qui montre des possibilités d'améliorer l'environnement scolaire pour les TJA.

Au niveau de l'école primaire, le fait de verser des incitations pour la participation des enseignants améliore la réussite des étudiants. Les incitations liées aux résultats d'examen des étudiants ont obtenu des résultats mitigés. Des études sont nécessaires pour déterminer si ces incitations peuvent augmenter les résultats des étudiants alors qu'ils commencent dans l'enseignement secondaire. Bien que les programmes scolaires et la pédagogie soient des sujets présentant un intérêt croissant, on ne sait pas quelles approches sont les plus efficaces. La fourniture de technologies de l'information et de la communication est prometteuse, mais seule une étude a examiné le potentiel des TIC sur l'apprentissage des étudiants. Cette étude a eu lieu au niveau de l'école primaire, et n'a pas montré d'amélioration de l'apprentissage des étudiants (Barrera-Osorio et Linden 2009). De toute évidence, davantage de recherches sont nécessaires pour étudier l'impact potentiel des TIC sur l'apprentissage.

\subsection{Programmes prometteurs, mais pour lesquels il est nécessaire d'obtenir davantage de preuves}

Les services de médecine préventive répondant aux besoins de santé particuliers des TJA filles et garçons sont quasiment inexistants dans les PRFM. En considérant la myriade de services sociaux et de santé dont les jeunes adolescentes et adolescents peuvent avoir besoin, le Population Council et ses partenaires ont développé un exemple de «Contrôle de santé» (voir le Tableau 5) qui expose les éléments sociaux et de santé clés pertinents pour ce groupe d'âge. Certains de ces éléments ont fait l'objet d'essais pilotes au Rwanda et ailleurs, mais une approche de «Contrôle de santé» complète doit encore être explorée.

L'expérimentation de certaines ou de l'ensemble de ces composantes, spécifiquement adaptées aux filles et aux garçons, est nécessaire et mérite un investissement. De tels efforts encourageraient des bilans de santé systématiques et la collecte de données de suivi sur la santé, et contribueraient à créer une «culture de la santé ». II s'agit d'une période potentiellement cruciale pour établir des comportements favorables à la santé et créer une demande de services de santé (Temin et Levine 2009).

\section{Promouvoir la santé mentale}

Afin de mieux assurer la transition saine des TJA vers l'âge adulte, les recherches ont décrit les facteurs de risque et de protection associés aux troubles mentaux chez les adolescents afin de guider les interventions (voir le Tableau 6) (Patel et al. 2007; Sawyer et al. 2012). Les preuves obtenues des examens systématiques ont démontré le potentiel des interventions de santé mentale pendant l'enfance ou l'adolescence. 


\section{TABLEAU 5 Les exemples d'éléments d'un « contrôle de santé » pour les TJA}

\section{doivent être adaptés aux filles et aux garçons}

\section{SANTÉ}

- Examen physique

- Examen des vaccinations et rappel

- Examen de nutrition/croissance

- Informations et services de santé sexuelle et reproductive

- Vaccin anti-VPH*

- Informations sur la prévention duVIH et du SIDA

- Dépistage de la violence et soutien

- Dépistage dans le domaine de la santé mentale et soutien

- Dépistage des blessures et prévention

- Vérification de l'activité physique/des jeux/ des sports

\section{SOCIAL}

- Conseils

- Renforcement des compétences nécessaires à la vie courante

- Évaluation pédagogique et soutien

- Dépistage et amélioration du soutien social offert par les pairs

- Dépistage de la consommation de drogues/d'alcool/de tabac et soutien pour la prévention des addictions

- Bien-être de la famille et soutien social

- Citoyenneté et renforcement des compétences de participation sociale, y compris l'acquisition de documents d'identité

Source : Catino 2012.

*REMARQUE: L'Organisation mondiale de la Santé recommande que les filles âgées entre 9 et 13 ans reçoivent le vaccin contre le papillomavirus humain (VPH). Même si, dans l'idéal, les filles comme les garçons devraient être vaccinés, étant donné les limitations liées au coût du vaccin et les difficultés de faire vacciner les garçons, le fait de cibler les filles et d'accroître la couverture pour les filles est une priorité.

TABLEAU 6 Les facteurs de risque et de protection associés à la santé mentale des adolescents

\begin{tabular}{|c|c|c|}
\hline & FACTEURS DE RISQUE & FACTEURS DE PROTECTION \\
\hline Biologiques & $\begin{array}{l}\text { Exposition aux toxines pendant la grossesse } \\
\text { Bagage génétique } \\
\text { Malnutrition } \\
\text { Dépression périnatale et post-partum } \\
\text { Autres maladies }\end{array}$ & $\begin{array}{l}\text { Programmes de soutien prénatal } \\
\text { Bonnes santé physique et nutritior } \\
\text { Soutien postnatal pour la mère }\end{array}$ \\
\hline Physiologiques & $\begin{array}{l}\text { Problèmes de développement et de comportement } \\
\text { Violence physique et émotionnelle } \\
\text { Négligence }\end{array}$ & $\begin{array}{l}\text { Amélioration de l'estime de soi } \\
\text { Compétences sociales } \\
\text { Capacité à résoudre les problèmes }\end{array}$ \\
\hline \multicolumn{3}{|l|}{ Sociaux } \\
\hline Famille & $\begin{array}{l}\text { Conflits familiaux } \\
\text { Soins inadéquats }\end{array}$ & $\begin{array}{l}\text { Attachement familial } \\
\text { Implication positive de la famille }\end{array}$ \\
\hline École & $\begin{array}{l}\text { Handicap éducatif } \\
\text { Environnement d'apprentissage inadéquat } \\
\text { Intimidation, harcèlement, victimisation }\end{array}$ & $\begin{array}{l}\text { Opportunités éducatives } \\
\text { Réussite scolaire gratifiante } \\
\text { Identité avec l'école }\end{array}$ \\
\hline Communauté & $\begin{array}{l}\text { Transitions (par ex., urbanisation, conflit, } \\
\text { déplacement, guerre) } \\
\text { Discrimination/marginalisation } \\
\text { Exposition à la violence }\end{array}$ & $\begin{array}{l}\text { Liens avec la communauté } \\
\text { Modèles positifs } \\
\text { Récompenses pour l'implication } \\
\text { au sein de la communauté }\end{array}$ \\
\hline
\end{tabular}

Source : Adapté de Patel et al. 2007. 
Un examen de 2013 des interventions de promotion de la santé mentale pour les jeunes âgés de 6 à 18 ans dans les PRFM a découvert des preuves de niveau modéré à élevé en faveur des interventions scolaires et communautaires concernant les résultats émotionnels et comportementaux (Barry et al. 2013). Sur les 22 interventions évaluées, les programmes inclus se concentraient sur les compétences nécessaires à la vie courante, la formation aux loisirs et à la résilience, les stratégies cognitives et comportementales, le renforcement des compétences liées à la famille, et les interventions psychosociales pour les enfants affectés par un conflit armé. Les résultats de l'examen ont mis en évidence le potentiel des programmes à composantes multiples, qui associent les parents et l'ensemble de la communauté pour fournir une formation scolaire sur les compétences sociales et émotionnelles. Dans certains cas, des effets différents ont été observés en fonction du genre, indiquant la nécessité d'effectuer davantage de recherches spécifiques au genre. Bien que cela n'ait pas été spécifiquement testé chez les adolescents, les preuves suggèrent également le potentiel de la délégation des tâches pour les services de santé mentale, y compris les interventions psychologiques pour la dépression périnatale, par des agents non spécialisés formés dans les PRFM (Patel et al. 2011; Chowdhary et al. 2014).

Bien que les résultats de ces études soient prometteurs, à l'échelle mondiale seules $10 \%$ des interventions de santé mentale ont été évaluées dans les PRFM, et les interventions incluant des adolescents étaient plus rares encore.

Pour guider les services de santé mentale appropriés sur le plan culturel et adaptés aux jeunes, il est nécessaire d'effectuer des recherches sur l'ampleur et les besoins spécifiques des TJA en matière de santé mentale à l'échelle locale. II est également nécessaire de renforcer les systèmes d'information liés à la maladie mentale. Un examen des États membres de l'OMS, par exemple, a découvert que peu de pays, et encore moins de pays en développement, disposaient d'un système de suivi des données des services de santé qui incluait la mesure de la santé mentale ( 8 sur 20 pays à haut revenu contre 1 sur 16 pays à faible revenu) (OMS, WPA et IACAPAP 2005). La mesure est également compliquée par la classification actuelle de la maladie mentale. Les normes actuelles s'appuient en général sur les critères DSM-V standard et sont basées sur des groupes de symptômes qui peuvent être observés chez une partie limitée de la population (Jacob et Patel 2014). Par conséquent, de nombreux adolescents qui présentent un risque de problèmes de santé mentale, mais qui ne montrent encore aucun symptôme clinique risquent de passer inaperçus.

Dans de nombreux PRFM, seule une faible proportion des adolescents ayant des problèmes de santé mentale reçoivent un traitement et un soutien appropriés (Morris et al. 2011). Les obstacles courants identifiés pendant un examen de 2012 de l'OMS sur les programmes de santé mentale pour les adolescents ont révélé que le manque de politiques gouvernementales ou nationales liées à la santé mentale entrave le développement de services, la responsabilisation et l'affectation des ressources (OMS 2012a). Les programmes de santé mentale existants identifiés pendant l'examen ne parvenaient souvent pas à atteindre les adolescents, n'étaient pas adaptés aux jeunes, étaient caractérisés par des coûts élevés à la charge des utilisateurs ou étaient planifiés en fonction de la mission d'une ONG ou d'une organisation, plutôt qu'en fonction des besoins de la population locale.

Le fait de promouvoir les besoins en santé mentale des très jeunes adolescents représente un défi à l'échelle mondiale, mais qui peut avoir un impact important sur la santé publique. Un nombre croissant de preuves montrent que les interventions réalisées tôt peuvent fournir des avantages à long terme sur la santé, pendant la transition des jeunes vers l'âge adulte. Des événements tels que la création du mouvement « Movement for Global Health », un forum de plaidoyer et de recherche en ligne, et du Programme d'action: Combler les lacunes en santé mentale de l'OMS, qui fournit des recommandations basées sur des preuves pour l'intensification des services dans les PRFM, révèlent une reconnaissance croissante de la pertinence de la santé mentale dans le développement à l'échelle mondiale (Patel, Boyce et al. 2011). 
La participation à des sports promeut la forme physique, permet de réduire les maladies chroniques et améliore la santé mentale en réduisant les symptômes du stress et de la dépression.

\section{Les avantages d'une bonne nutrition, de l'activité physique et des sports}

Il a été prouvé que les sports et l'activité physique ont des effets sociaux et mentaux positifs tout au long de la vie. Par exemple, les avantages des sports pour la santé physique et mentale sont appuyés par de nombreuses recherches: la participation régulière à des sports promeut la forme physique, permet de réduire les maladies chroniques et améliore la santé mentale en réduisant les symptômes du stress et de la dépression (CDC 2015). Des recherches de plus en plus nombreuses soutiennent la conclusion importante qu'un mode de vie actif entraîne une baisse du risque de maladies cardiaques, de certains cancers, de l'obésité et de l'ostéoporose (Staurowsky et al. 2015; OMS 2015; CDC 2015). En2004, l'Organisation mondiale de la Santé a proposé une «Stratégie mondiale pour l'alimentation, l'exercice physique et la santé» afin de catalyser l'action en vue d'améliorer l'alimentation et augmenter l'activité physique à l'échelle des pays. Plus récemment, l'OMS a adopté un Plan d'action mondial pour la lutte contre les maladies non transmissibles (MNT) 2013-20 qui comprend des indicateurs clés liés au régime alimentaire et à l'activité physique (Puska 2004). Ensemble, ces efforts sont conçus pour réduire les taux de maladie et de décès liés à une mauvaise alimentation et à un manque d'activité physique.

Une bonne nutrition et une activité physique adéquate sont des comportements modifiables qui peuvent endiguer l'incidence croissante des MNT observées dans les PHR et les PRFM (voir la Section2.2). La hausse à l'échelle mondiale du surpoids chez les jeunes est préoccupante, car les enfants et les jeunes adolescents obèses sont susceptibles de devenir des adultes obèses et de souffrir d'une mauvaise image d'eux-mêmes qui peut interférer avec un développement positif. Un examen des interventions liées à l'obésité dans le contexte scolaire desPRFM a souligné le potentiel des interventions qui ciblent les comportements alimentaires et/ou l'activité physique chez les enfants et les adolescents. Les résultats suggèrent que les interventions qui ciblent à la fois l'alimentation et l'activité physique, incluent des modèles parentaux pour un comportement sain et intègrent des activités éducatives dans le programme scolaire peuvent améliorer la santé physique des adolescents, bien que peu d'études examinées aient présenté des résultats anthropométriques (Verstraeten et al. 2012; Hingle et al. 2010). Les interventions scolaires qui incluent des programmes basés sur les compléments alimentaires, comme la fortification en fer, en plus de l'éducation à la diversité alimentaire, peuvent être particulièrement efficaces pour améliorer les résultats nutritionnels, comme réduire la prévalence de l'anémie, qui préoccupe particulièrement les filles enceintes (voir la Section2.3) (Black et al. 2013).

Les sports et les jeux sont également associés au développement sain de l'enfant et à l'efficacité personnelle, et semblent avoir des effets thérapeutiques après un traumatisme. De plus en plus, les sports sont utilisés comme des plateformes pour délivrer des campagnes et des messages de santé; ceux-ci sont rares à avoir été rigoureusement évalués (Clark et al. 2006). Malgré les preuves attestant clairement que les sports et l'activité physique promeuvent une bonne santé, les normes sociales et culturelles empêchent les femmes et les filles de faire du sport. Cependant, l'émergence récente de nouvelles opportunités sportives pour les filles représente un éloignement des scénarios traditionnels associés à la féminité (Brady 2011a).

La pratique sportive permet de transformer la perception que les filles ont d'elles-mêmes et la manière dont leur famille et leur communauté les perçoivent, ce qui peut contribuer à une évolution des normes de genre. L'entrée et la visibilité des filles dans l'arène publique remet en question les mythes culturels des comportements acceptables en fonction du genre. La participation aux sports permet aux filles d'accéder au réseau d'institu- 
tions, de programmes, de mentors et de modèles auxquels elles n'auraient autrement pas accès. Ces connections sont extrêmement précieuses et manquent souvent aux filles. II a été démontré que ce capital social a des avantages innombrables pour les filles, y compris en tant que facteur de protection contre les rapports sexuels forcés (Hallman 2011).

La fin de l'enfance et le début de l'adolescence (10-12 ans) est un moment où les filles bénéficient généralement des libertés accordées aux garçons en matière de mobilité et d'activités physiques avant que de nombreuses restrictions liées au genre n'apparaissent. Les normes sociales concernant la mobilité des filles et des garçons, les activités de loisir et l'accès à l'espace public commencent à devenir évidentes autour de la puberté; les préoccupations en matière de sécurité pour les filles sont exacerbées, et leur mobilité est donc souvent restreinte (Hallman et al. 2014). Les recherches suggèrent qu'un engagement précoce dans les sports et l'activité physique est important, en particulier pour les filles; si elles n'y participent pas avant le début de l'adolescence, elles sont moins susceptibles d'y participer plus tard au cours de leur vie. La période du début de l'adolescence est un moment opportun pour engager les TJA dans les sports et l'activité physique, qui fournit des avantages immédiats et à long terme.

\section{Les TJA et les technologies de l'informa- tion et de la communication (TIC)}

Les TIC ont le potentiel d'éduquer et d'offrir davantage d'autonomie aux jeunes adolescents, car les TJA sont souvent des «adeptes précoces » des nouvelles technologies (Raftree et Bachan 2013). Par exemple, une analyse des pays disposant de données indique que les jeunes de moins de 25 ans sont plus susceptibles d'utiliser Internet par rapport à ceux de 25 ans et plus (UNICEF 2012c).

Un nombre croissant de recherches, bien que limitées, s'efforcent d'examiner de manière systématique l'efficacité des interventions recourant aux TIC pour améliorer les résultats des adolescents. La plupart de ces recherches ont été menées dans les pays à haut revenu (Guse et al. 2012; Lau et al. 2011). Les résultats des examens systématiques qui se sont concentrés sur les interventions basées sur les TIC chez les adolescents ont, jusqu'à présent, été mitigés et ont eu différents résultats. Par exemple, une étude réalisée au Brésil et au Kenya a réparti de manière aléatoire des écoles pour inscrire les étudiants, soit dans une intervention en ligne sur la santé sexuelle, soit en leur fournissant un accès aux outils électroniques sans Internet (Halpern et al. 2008). Lors du suivi, on a constaté des différences importantes en matière de connaissances et d'attitudes entre les groupes d'intervention et de comparaison; cependant, le degré de changement était faible et l'effet obtenu ne suivait pas toujours la direction anticipée.

De plus, certains programmes basés sur les TIC ciblant les adolescentes ont rencontré une résistance des parents et de la communauté concernant les préoccupations liées aux normes sociales sur les comportements appropriés pour les filles, malgré l'efficacité prouvée de ces programmes. Par exemple, dans un effort d'améliorer l'alphabétisation chez les adolescentes au Pakistan, les filles ont reçu un téléphone portable avec une connexion prépayée et ont reçu des messages SMS des enseignants sur les sujets liés à la santé, à la nutrition et à la religion. Après des efforts consacrés à éduquer les familles quant à la nature des SMS et leurs avantages pour l'alphabétisation, le programme a été davantage accepté, indiquant que les interventions basées sur les TIC ont le potentiel d'influencer le changement social à de multiples niveaux (GSM Association 2013b). Les autres interventions basées sur les TIC et visant la population dans son ensemble, comme l'initiative « Half the Sky Movement Media and Technology Engagement Initiative », montrent également le potentiel de promouvoir l'égalité entre les sexes, par le biais de films, d'outils éducatifs en ligne et de jeux éducatifs mobiles (Dasgupta et al. 2012).

Bien que les résultats des interventions basées sur les TIC soient prometteurs, davantage de recherches tirées d'études rigoureuses sont nécessaires pour construire une base de preuves solides sur l'efficacité des TIC en général, et en particulier concernant leur utilisation par les TJA. De plus, des stratégies fondées sur des preuves sont nécessaires pour guider et aider les jeunes adolescents à utiliser les TIC de manière appropriée. En équipant les adolescents pour qu'ils de- 
viennent des utilisateurs informés des TIC, les TJA peuvent mieux éviter les risques comme le contenu inadapté sur Internet, les comportements de prédateurs en ligne, et le partage involontaire des informations privées (Raftree et Bachan 2013).

Pour assurer une application complète, des stratégies parallèles sont nécessaires pour lutter contre les obstacles tels que les normes de genre et les coûts, pour que les adolescents les plus défavorisés puissent en bénéficier.

\section{Les programmes répondant aux TJA dans des circonstances graves et particulières}

\section{Contextes humanitaires, de crise et d'urgence}

II semble que les TJA se voient souvent imposer certaines activités pendant les crises (CAG 2012). Alors même que les adolescents assument davantage de responsabilités pour assurer la survie des membres de leur famille, les adultes et le personnel humanitaire ne reconnaissent pas l'énormité de leurs actions pour renforcer la santé et le bien-être social de leur famille pendant la crise. Comme c'est souvent le cas des travaux non rémunérés, les soins et les activités productives dans lesquelles les TJA s'engagent les cachent aux yeux du public, ce qui les rend invisibles pour les acteurs chargés de l'intervention d'urgence. En raison de ce manque de visibilité, les programmes d'intervention humanitaire, tels qu'ils sont conçus actuellement, négligent en général les jeunes adolescentes et adolescents en tant que populations distinctes.

Les efforts d'intervention d'urgence parviennent rarement à répondre aux besoins des très jeunes adolescentes, par rapport à ceux des très jeunes adolescents. Certaines activités de secours négligent involontairement les adolescentes, par exemple, en partant du principe que leur liberté de mouvement au sein de la communauté ou du camp est identique à celle des garçons ou des femmes adultes (CAG 2012). Les recherches ont montré que même en dehors des situations de crise, les préoccupations pour la sécurité limitent fortement l'accès spatial des filles pubères au sein de la communauté par rapport aux garçons (Hallman et al. 2014). Des espaces sûrs réservés aux filles et aux garçons dans les contextes humanitaires sont donc étudiés en tant qu'outils de programmation (Browne 2013).

Le renforcement économique (l'éducation financière, la formation professionnelle et le micro-crédit) est examiné en tant que mécanisme pour réduire le risque de violences basées sur le genre pour les jeunes adolescents dans les contextes humanitaires (Caton et al. 2014). Une étude récente réalisée dans trois pays par la Commission des femmes pour les réfugiés (WRC) et Johns Hopkins University a conclu qu'il est essentiel d'impliquer les parents, les pairs, les amis, les enseignants et les prestataires de santé dans le renforcement des connaissances et la sensibilisation aux besoins et aux risques des TJA dans les contextes humanitaires (WRC et al. 2014).

L'évaluation de la politique et des programmes de secours d'urgence s'est, jusqu'à aujourd'hui, peu concentrée sur les besoins des très jeunes adolescents et adolescentes en tant que populations particulières. Cependant, cela est en train d'évoluer. L'étude WRC-JHU (WRC et al. 2014) a discuté avec les TJA en Éthiopie, au Liban et en Thaillande des connaissances, risques et besoins concernant la santé sexuelle et reproductive. Un consortium d'agences de développement et de secours a formé le «Groupe de travail sur les adolescentes dans les situations d'urgence» pour établir des priorités en matière de programmes et de recherches pour les filles dans des contextes d'urgence. Ce groupe postule qu'il existe en fait peu d'outils programmatiques ou de recherche pertinents pour les besoins des TJA dans les situations d'urgence. Les partenaires du consortium ont récemment identifié cinq phases dans le cycle d'intervention à une situation d'urgence dans lesquelles les besoins varient en fonction du genre et de l'âge. Parmi les rares recherches existantes sur les TJA dans les situations d'urgence, la plupart se concentre sur les périodes de stabilisation et de reconstruction plus tardives. Les interventions programmatiques pour les jeunes adolescentes et adolescents doivent être élaborées pour les phases précoces du cycle également.

Les bailleurs de fonds et les acteurs au sein du monde humanitaire doivent évaluer les défis 


\section{UNE NOUVELLE APPROCHE ÉLABOREÉ PAR LE GROUPE DE TRAVAIL SUR LES ADOLESCENTES DANS LES SITUATIONS D'URGENCE}

L'approche « I'm here ॥ [Je suis ici] pour les recherches orientées vers l'action implique les filles qui se trouvent dans les situations d'urgence. Des outils sont inclus pour accompagner chaque action (Robles 2014).

Dans un contexte de crise, les programmes devraient:

- Identifier la communauté spécifiquement affectée par la crise où des adolescentes déplacées sont concentrées et cartographier les principaux points de service où les acteurs humanitaires fournissent des informations et des services d'urgence.

- Rendre l'univers des adolescentes visible: les trier en fonction de catégories de vulnérabilité et de capacité de base, par ex., l'âge, la situation matrimoniale, l'éducation, le statut en matière d'accompagnement et de grossesse.

- Organiser des réunions de groupe avec les adolescentes ayant des vulnérabilités ou des capacités similaires pour connaître les besoins urgents, les peurs et les préoccupations concernant la protection des filles, et pour enregistrer les informations vitales, les compétences et les atouts dont elles ont besoin pour surmonter les conséquences négatives du déplacement et pour réduire leur risque de connaître la violence.

- Élaborer des plans spécifiques qui répondent à l'univers des filles dans la zone affectée par la crise, par ex., mettre en place des espaces physiques sûrs où les filles peuvent immédiatement apprendre et recevoir des informations et des services vitaux et, dès que possible, bénéficier d'un soutien ciblé axé sur le renforcement des atouts.

- Rassembler les secteurs humanitaires et les acteurs locaux sur la nécessité de mettre en place des interventions d'urgence, des stratégies, des indicateurs et des droits adaptés aux adolescents.

- Mettre à contribution la capacité des adolescentes à soutenir les interventions humanitaires et les opérations de secours.

spécifiques que rencontrent les adolescentes et les adolescents dans les situations d'urgence et développer des recommandations concrètes spécifiques à chaque sexe. Fournir aux TJA des services accessibles, des espaces sûrs et un soutien permettrait non seulement de réduire leurs risques, mais également d'entraîner une réaction globale plus solide, en particulier compte tenu des responsabilités invisibles assumées par les jeunes adolescents pour leur famille. Le fait d'identifier et de concevoir des programmes réduisant largement ces fardeaux importants mais non reconnus pourrait diminuer la probabilité qu'ils connaissent des déficits graves et irréversibles, de santé, psychosociaux et d'éducation.

\section{Migrants volontaires : des conseils sont disponibles pour les migrants internes, mais sont insuffisants pour les cas de migration transfrontalière}

Les jeunes adolescents sont affectés par la migration dans toutes les régions du monde, mais la compréhension de ses effets est très limitée. La collecte des données, le suivi et la recherche sont nécessaires pour mieux comprendre comment la migration affecte les sociétés, les familles et les adolescents dans les pays d'origine et d'installation; pour étayer les politiques en vue d'atténuer les impacts négatifs, et pour permettre aux familles et aux enfants de prendre des décisions 
LES FILLES EN DÉPLACEMENT : PROGRAMME D'ACTION POUR AMÉLIORER LA VIE DES ADOLESCENTES MIGRANTES

1. Préparer et équiper les filles avant leur migration : Assurer l'éducation, les compétences nécessaires à la vie courante, la connaissance des droits, les documents d'identité.

\section{Adapter les services de santé et} d'éducation aux filles migrantes: S'assurer que les prestataires de services prennent en compte l'âge, le sexe et le statut en matière de migration.

3. Assurer une arrivée en douceur pour les filles migrantes : Réduire l'isolement par des lieux sûrs où elles peuvent séjourner et des liens avec des personnes de confiance.

4. Mettre en place un filet de sécurité : Réserver le temps et l'espace nécessaires pour que les filles migrantes rencontrent leurs pairs, leurs mentors et leurs réseaux de soutien.

5. Tester des manières innovantes de préparer la réussite des filles migrantes : Examiner les moyens de développer les atouts des filles avant que les choses ne tournent mal.

6. Se concentrer sur les plus isolées et vulnérables: Concevoir des approches réservées aux filles pour atteindre les employées de maison, les épouses enfants et les filles exploitées sexuellement.

7. Combler les lacunes importantes en matière de preuves : Éclairer les taux de migration interne ventilés par âge et par sexe en utilisant des données quantitatives nouvelles et existantes. Développer des études qualitatives et longitudinales pour faire la lumière sur les expériences des filles migrantes, et pour évaluer et améliorer les programmes.

\section{Accroître la visibilité des filles mi-} grantes par le biais des politiques et du plaidoyer : Maximiser les avantages de la migration pour les filles adolescentes en augmentant leur visibilité dans l'engagement politique et les efforts de plaidoyer. informées à propos du déplacement. L'étude «Girls on the Move» (Temin et al. 2013) fournit aux décideurs politiques, aux planificateurs de programmes et aux bailleurs de fonds des preuves sur les circonstances des filles migrantes internes, et offre huit recommandations pour réduire au maximum les aspects négatifs de la migration et maximiser ses avantages pour les filles adolescentes dans le monde en développement.

Les TJA qui migrent à l'échelle internationale sont confrontés à des difficultés supplémentaires. Beaucoup d'entre eux traversent les frontières sans permission légale et deviennent des non-citoyens. La plupart des cadres juridiques existants ne traitent pas directement cette conséquence. Les lois nationales de protection des enfants ne couvrent pas les questions de citoyenneté étrangère, notamment le risque de déportation et le manque de droits aux avantages sociaux auxquels sont confrontés les enfants non-citoyens. De plus, la loi sur la migration, qui établit les paramètres du statut juridique pour les catégories reconnues de migrants, ne contient pas de dispositions pour les besoins et les circonstances de la plupart des enfants et des adolescents qui voyagent indépendamment de leur famille (Bhabha 2008).

La législation régionale et nationale qui réglemente l'immigration a inclus des dispositions promouvant l'unité des familles et le droit des mineurs à rejoindre leurs proches adultes. Cependant, un engagement plus large concernant les migrants mineurs qui traversent les frontières est nécessaire. Il existe peu de législations internationales ou régionales qui traitent de manière systématique et complète les besoins des mineurs. Par conséquent, la législation existante est réputée incohérente et incomplète concernant les enfants migrants internationaux, y compris les très jeunes adolescents (Bhabha 2008).

\section{Les adolescents victimes de la traite : les prestataires de soins de santé peuvent être des alliés clés pour identifier et aider les victimes}

Le fait de doter les prestataires de soin des connaissances et des compétences nécessaires représente un autre domaine d'intervention prometteur. Les études suggèrent que jusqu'à 
la moitié des victimes du trafic sexuel aux ÉtatsUnis (la plupart venues de l'étranger) obtiennent des soins médicaux alors qu'elles sont victimes du trafic. Cependant, la manière dont le système de soins de santé répond aux besoins des victimes du trafic sexuel n'est pas claire. Les services de santé pour les victimes sont incomplets et mal coordonnés, en particulier dans le domaine de la santé mentale (Konstantopoulos et al. 2013; Baldwin et al. 2011; Family Violence Prevention Fund et World Childhood Foundation 2005). Divers facteurs fonctionnent comme des obstacles à une meilleure réponse en matière de santé, y compris le manque de sensibilisation au trafic sexuel et les préjugés des agents de santé. Une réponse plus complète et coordonnée du système de santé face au trafic sexuel pourrait permettre d'atténuer ses effets dévastateurs sur les femmes et les filles vulnérables.

II n'existe aucun outil de dépistage validé cliniquement pour les jeunes adolescents, les adolescents plus âgés ou les adultes qui soit spécifiquement conçu pour identifier les victimes dans le milieu médical. Étant donné que ces personnes s'identifient rarement en tant que victimes, la majorité d'entre elles ne sont probablement pas reconnues (Greenbaum 2014). L'opportunité d'une évaluation et d'une intervention complète est ensuite perdue.
Les professionnels de la santé peuvent œuvrer en vue d'améliorer le dépistage, l'identification et l'assistance des victimes du trafic sexuel dans un contexte clinique et aider ces femmes et ces filles à accéder aux services juridiques et sociaux. Les prestataires de soins de santé représentent l'un des rares groupes de professionnels qui peuvent entrer en contact avec les victimes du trafic sexuel. Les professionnels de la santé peuvent œuvrer en vue d'améliorer le dépistage, l'identification et l'assistance des victimes du trafic sexuel dans un contexte clinique et aider ces femmes et ces filles à accéder aux services juridiques et sociaux. 



\section{Les recherches auprès des TJA: recueillir des informations pour guider les programmes et les politiques}
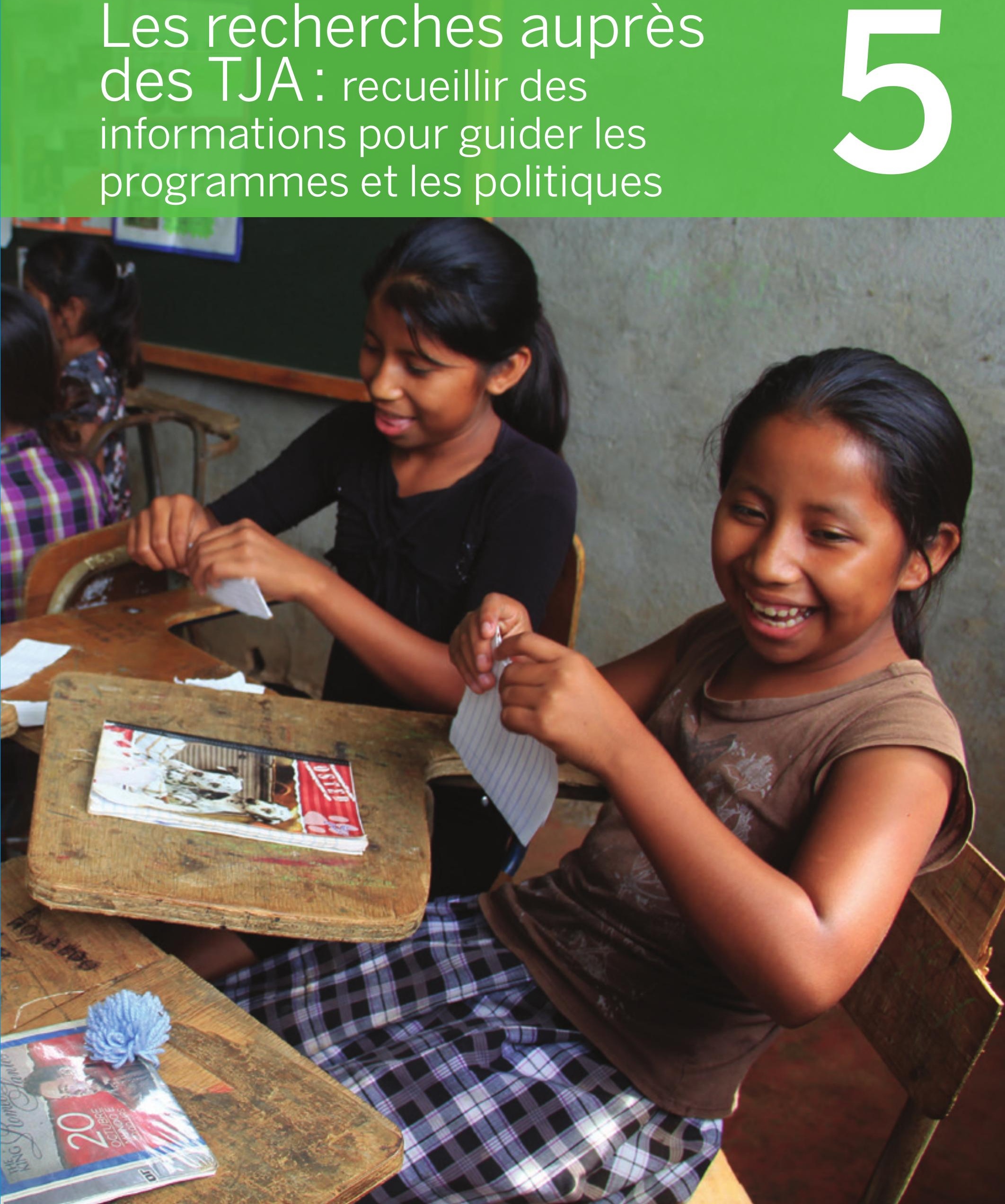


\section{1 Études incluant les données disponibles sur les TJA}

Des recherches sur les jeunes adolescents apparaissent, mais nécessitent encore davantage d'innovation, de tests et d'application. Sans ces recherches méthodologiques, on continuera à manquer d'informations sur les risques, les besoins et les capacités de ce groupe d'âge. Dans cette section, nous mettons en évidence les données actuelles et discutons des défis éthiques et méthodologiques que nous rencontrons lorsque nous recueillons des données sur les TJA. Nous commençons par souligner les principaux programmes d'enquête incluant des données sur les TJA. Nous fournissons ensuite une vue d'ensemble des considérations éthiques et mettons en évidence les documents d'orientation disponibles pour ceux qui envisagent d'inclure des TJA dans leurs recherches. Enfin, nous présentons une vue d'ensemble des méthodes et outils qui pourraient être utilisés avec les TJA dans une étude de recherche.

Certaines informations concernant les TJA sont disponibles dans des ensembles de données existants sur le développement et la santé infantiles, ou peuvent être intégrés dans la fourchette d'âge plus jeune des enquêtes portant sur les adolescents et les jeunes. II convient de noter que les données concernant la vie des jeunes adolescents sont limitées et ne sont pas réunies en un endroit; elles doivent être récupérées à partir de diverses sources. Cependant, un certain nombre de programmes d'enquête incluent les TJA dans leurs recherches.

\section{Programmes d'enquête axés principale- ment sur le groupe d'âge des TJA}

Des exemples de programmes d'enquête majeurs qui recueillent des données auprès des jeunes adolescents eux-mêmes sont présentés dans l'Encadré 1. Les programmes d'enquête, comme les enquêtes sur la violence contre les enfants (VACS) de I'UNICEF/CDC, qui posent des questions sensibles liées aux expériences de violence (voir l'Encadré 2) ont été efficaces en grande partie grâce à l'engagement important et à la réactivité des parties prenantes vis-à-vis des comités d'éthique de chaque pays visé. Aux États-Unis, des études ont été menées pendant lesquelles des enfants dès l'âge de 10 ans ont été interrogés à propos de la violence sexuelle (Finkelhor et al. 2005). De plus, l'outil de dépistage de la violence envers les enfants de l'ISPCAN a été utilisé dans plusieurs pays pour recueillir des données sur l'étendue et la nature de la maltraitance chez les enfants âgés de 12 à 17 ans (Zolotor et al. 2009). Ces outils nécessitent une adaptation locale et des essais préliminaires au sein d'un petit groupe de répondants ayant des caractéristiques similaires à la population étudiée.

\section{Les programmes d'enquête réalisés auprès de répondants ne faisant pas partie des TJA, mais qui incluent des informations sur les TJA}

Un grand nombre de grandes initiatives d'enquête sont de bonnes sources d'informations à propos des TJA, rapportées par un répondant adulte du ménage. La plupart de ces initiatives incluent des données sur la santé, l'anthropométrie, l'éducation et le travail chez les TJA, entre autres sujets. De telles enquêtes incluent, sans s'y limiter, les enquêtes démographiques et de santé(EDS), les enquêtes de surveillance comportementale sur le VIH/SIDA/IST, les enquêtes en grappes à indicateurs multiples (MICS) de I'UNICEF, et les mesures des niveaux de vie (Living Standards Measurement Surveys - LSMS) de la Banque mondiale.

\subsection{Considérations éthiques dans les recherches effectuées directement auprès des TJA}

Bien que les données d'enquête décrites cidessus fournissent certaines informations sur le groupe d'âge concerné, elles ne couvrent pas de manière exhaustive tous les sujets ou régions. Il peut donc être nécessaire de recueillir des données primaires auprès des TJA. La prudence de la plupart des chercheurs, dans leur décision d'inclure ou non les TJA dans leurs études, est justifiée. Le manque de compréhension, les questions d'éthique et de sécurité, l'approbation 


\section{ENCADRÉ 1 Programmes d'enquête majeurs présentant des données de répondants de}

plus de 14 ans qui comprennent des informations sur les TJA

Enquêtes démographiques et de santé (EDS). Le programme d'EDS recueille des données représentatives à l'échelle nationale sur la santé, la population, le VIH/SIDA et la nutrition dans plus de 90 PRFM. Les ensembles de données disponibles tirées des EDS comprennent des données d'enquête auprès des ménages, qui incluent des indicateurs sur les résidents de tous âges du ménage. En plus des enquêtes sur les ménages, des enquêtes individuelles sont menées avec des femmes de 15 à 49 ans, et des hommes âgés de 15 à 49 ans, de 15 à 54 ans ou de 15 à 59 ans. Les données présentées sur les adolescents et les jeunes sont tirées des enquêtes sur les ménages (groupe d'âge 10-14 ans) et des enquêtes individuelles (plus de 15 ans). Les données sont pondérées pour être représentatives à l'échelle nationale, urbaine/rurale et régionale. Pour plus d'informations, veuillez consulter: www.dhsprogram.com/data/.

Comportements de santé des enfants d'âge scolaire (Enquête HBSC). L'enquête HBSC recueille des données sur les jeunes d'âge scolaire de 11, 13 et 15 ans en utilisant des questionnaires remplis par les élèves euxmêmes et administrés dans la classe. L'adhésion est actuellement limitée aux pays de la région européenne; 36 pays y ont participé pendant la série de collecte des données de 2001-02. La série de questions de base examine les facteurs liés au contexte, les ressources individuelles et sociales, les comportements en matière de santé et les résultats sur la santé. Pour plus d'informations, veuillez consulter: www.hbsc.org.

Les enquêtes de surveillance comportementale (ESC) sur le VIH/SIDA/IST. L'ONUSIDA, I'OMS, I'organisation Family Health International et d'autres agences ont travaillé au développement d'un cadre pour la surveillance du VIH qui est adapté au stade de l'épidémie au sein des pays. Les données des ESC suivent les tendances concernant les connaissances, les attitudes et les comportements à risque sur le VIH/SIDA dans les sous-populations ayant un risque particulier d'infection au $\mathrm{VIH}$, comme les travailleuses du sexe, les utilisateurs de drogues injectables, les hommes migrants et les jeunes, et ont été mises en œuvre dans 15 pays d'Afrique et d'Asie. Bien que les moins de 15 ans ne fassent généralement pas partie des populations de jeunes interrogés, les questions rétrospectives de l'ESC produisent des données importantes concernant les comportements au début de l'adolescence. Pour plus d'informations, veuillez consulter: www.fhi.org.

La mesure des niveaux de vie (Living Standards Measurement Surveys - LSMS). L'enquête LSMS est un programme d'enquête auprès des ménages réalisé par la Banque mondiale, qui recueille des données sur les sujets liés à la pauvreté, l'éducation, la migration, les revenus, l'agriculture, la santé et la fertilité. En plus des informations sur le statut du ménage, les répondants adultes du ménage (âgés de 15 ans et plus) fournissent des informations sur l'éducation et la santé des enfants et des jeunes adolescents. Les enquêtes ont été mises en œuvre dans 36 PRFM depuis 1985. Pour plus d'informations, veuillez consulter: http://go.worldbank. org/IPLXWMCNJO.

Les enquêtes en grappes à indicateur multiple (MICS). Les enquêtes MICS sont un programme d'enquête auprès des ménages élaboré par I'UNICEF pour aider les pays à combler les lacunes de données pour le suivi de la situation des enfants et des femmes. Le site web MICS fournit un accès aux fichiers de données MICS, aux enquêtes, aux outils et aux ressources, y compris près de 250 enquêtes dans plus de 100 pays. L'enquête MICS est composée de trois questionnaires: un questionnaire pour les ménages, un questionnaire pour les femmes âgées de 15 à 49 ans, et un questionnaire concernant les enfants de moins de 5 ans. Bien que les enquêtes MICS n'interrogent pas directement les très jeunes adolescents, comme les enquêtes EDS, elles produisent des données rétrospectives riches et sont une source d'informations importantes sur les adolescents déscolarisés. Consultez le site web MICS sur www.childinfo.org.

L'Institut de statistique de I'UNESCO (ISU). L'ISU recueille des données transversales et comparables sur le plan national sur l'éducation, l'alphabétisation, la science, la technologie, la culture et la communication pour plus de 200 pays et territoires, sur une base annuelle ou bisannuelle. Les données recueillies sont disponibles pour les enfants qui sont en âge d'être à l'école primaire ou plus âgés, qui sont à l'école ou déscolarisés, ainsi que les adultes. Les questionnaires et les fichiers de données de l'ISU sont diffusés sur le centre de données de I'ISU, ainsi que sur le rapport des Nations unies sur les objectifs du Millénaire pour le développement et le Rapport mondial de suivi sur l'éducation pour tous. Pour plus d'informations, veuillez consulter: http://www.uis.unesco.org/UISQuestionnaires/Pages/defaultFR.aspx?SPSLanguage=FRwww.uis. unesco.org/UISQuestionnaires/. 
ENCADRÉ 2 Programmes d'enquêtes majeurs axés principalement sur le groupe d'âge des TJA

L'enquête mondiale réalisée en milieu scolaire sur la santé des élèves (GSHS). Fruit d'une collaboration entre l'OMS et les CDC, l'enquête GSHS est un projet de surveillance conçu pour aider les pays à mesurer et évaluer les facteurs de risques comportementaux et les facteurs de protection dans dix domaines clés chez les jeunes âgés de 13 à 15 ans. Les dix domaines sont: la consommation d'alcool et d'autres drogues; les comportements alimentaires; I'hygiène; la santé mentale; l'activité physique; les facteurs de protection; les renseignements démographiques; les comportements sexuels; la consommation de tabac; et la violence et les blessures involontaires. Vingt-deux pays dans toutes les régions du monde ont soit déjà mis en œuvre l'enquête GSHS, soit sont en train de le faire. Pour plus d'informations, et pour obtenir des fiches d'informations sur les pays et des questionnaires, consultez: www.who.int/ school_youth_health/assessment/gshs/en/.

L'enquête sur la violence contre les enfants (VACS). L'UNICEF, en collaboration avec les CDC et les partenaires gouvernementaux, effectue des enquêtes nationales basées sur la population de plusieurs pays d'Afrique de l'Est et du Sud et des Caraïbes pour générer des preuves sur les violences sexuelles et d'autres types de violence contre les enfants. Les recherches des enquêtes VACS s'étendent désormais aux pays de la région de l'Asie de l'Est et du Pacifique. Les recherches des enquêtes VACS recueillent des données sur la prévalence de la violence physique, émotionnelle et sexuelle contre les enfants, ainsi que les facteurs de risque et de protection associés à la violence et ses conséquences sur la santé. Des enquêtes représentatives à l'échelle nationale de jeunes femmes et hommes âgés de 13 à24 ans sont effectuées en utilisant une enquête des ménages en trois étapes pour s'assurer que les données soient représentatives aux niveaux régional et sous-régional. Pour plus d'informations, veuillez consulter: www.cdc.gov/violenceprevention/ vacs/publications.html.

Young Lives : Une étude internationale sur la pauvreté infantile. Young Lives est une étude longitudinale d'une durée de 15 ans conçue pour améliorer la compréhension des causes et des conséquences de la pauvreté infantile. Elle inclut 12000 enfants de quatre pays: l'Éthiopie, I'Inde, le Pérou et le Vietnam. Par le biais d'une enquête auprès des ménages, deux cohortes sont suivies dans chaque pays: les enfants nés en2001-02 et en 1994-95. Des entretiens approfondis et des études de cas sont également effectués de manière à compléter les données recueillies sur les circonstances matérielles et sociales où les enfants résident, en ajoutant des informations sur les perspectives et les aspirations des jeunes. Pour plus d'informations, veuillez consulter: www.younglives.org.uk/what-we-do/access-our-data/access-our-data.

des comités d'examen internationaux, ainsi que la possibilité d'avoir des taux élevés de non-réponse sont des préoccupations (Chong, Hallman et Brady 2006). La peur, la stigmatisation, la honte et le biais de désirabilité sociale peuvent rendre invalides les réponses des TJA aux questions sur des comportements sensibles comme l'activité sexuelle ou les expériences de violence. Les déclarations rétrospectives par un groupe plus âgé (par ex., les 15-19ans) sur leurs comportements et leurs expériences alors qu'ils étaient des TJA ont souvent été utilisées, même si les biais de rappel sont une source de préoccupation. Le problème de cette approche est qu'elle n'apprend rien de la situation actuelle des TJA. L'Encadré 3 présente des questions favorisant la réflexion au moment de décider d'inclure ou non les TJA pour qu'ils répondent directement à l'étude.

\section{Quelles sont les ressources éthiques disponibles?}

Dans les cas où la collecte de données auprès des TJA semble justifiée, des mesures rigoureuses doivent être prises pour réduire au maximum les dangers potentiels pour les participants à l'étude. Des documents d'orientation sont disponibles et s'appuient sur les recherches dans les domaines 
d'étude de la protection des enfants, de la violence sexuelle et de la population. Ceux-ci comprennent:

- Recherche éthique impliquant des enfants (ERIC), un guide des approches éthiques pour l'inclusion des enfants dans les études internationales (Graham et al. 2013).

- Groupe de référence sur le suivi et l'évaluation de la protection de l'enfant, sur le fait de réduire l'inconfort, l'angoisse et le traumatisme des enfants ayant survécu à des violences (CPMERG 2012)

- Ethical Approaches to Gathering Information from Children and Adolescents in International Settings (Approches éthiques à la collecte d'information auprès des enfants et adolescents dans des contextes internationaux) (Schenk et Williamson 2005).

- WHO Ethical and Safety Guidance Notes for Research with Adolescents (Notes d'orientation de l'OMS sur l'éthique et la sécurité pour les recherches avec des adolescents (OMS 2011).

- Ethics Review Committee documentation (Documentation du Comité d'examen éthique) sur la recherche portant sur la santé reproductive dans les PRFM (Marie Stopes International, documents du Comité d'examen éthique).

Les études réalisées avec les TJA doivent être examinées par des Comités d'examen indépendants, à la fois dans le pays où l'étude aura lieu et avec les institutions d'accueil des partenaires de recherche. Il est également recommandé d'impliquer les groupes nationaux de protection des enfants et les organisations locales de la société civile travaillant sur la protection des enfants pour passer en revue les outils d'étude et fournir des conseils pour indiquer si des questions particulières sont susceptibles de causer de l'angoisse et si elles doivent être modifiées ou posées avec une prudence extrême aux TJA.

\section{Protéger les TJA répondants pendant le processus de recherche}

Pour réduire au maximum le risque de créer de l'angoisse chez les répondants, les attitudes du personnel de recherche concernant les sujets de recherche (le sexe chez les TJA, la maltraitance des enfants, la violence sexuelle à l'encontre des enfants, etc.) devraient être examinées dans le cadre de leur formation et dans le cadre de la décision finale concernant les personnes qui
ENCADRÉ 3 Les recherches devraient-elles inclure les TJA?

Pour décider du sujet principal qui sera exploré au cours d'une étude, les chercheurs devraient clarifier si des questions aux TJA sont nécessaires. La question nécessite-t-elle l'implication directe ou indirecte des TJA ? Chaque question est-elle appropriée ? Les participants peuvent-ils être affectés? En particulier lorsque les recherches posent des questions sur les expériences d'exploitation ou de violence, les répondants peuvent-ils être traumatisés davantage en répondant aux questions, ou pourrait-il y avoir des représailles des adultes dont les actions ont été signalées par l'enfant?

- En définissant l'échantillon, les chercheurs devraient clarifier si les TJA peuvent être blessés parce qu'ils sont inclus dans l'échantillon ou parce qu'ils en sont exclus.

- En recueillant des données, les chercheurs devraient clarifier s'il existe des risques physiques, psychologiques, sociaux ou émotionnels potentiels pour les sujets ou les proches des sujets. Quelles sont les meilleures méthodes de recherche à utiliser? Quelle est la meilleure personne pour approcher les TJA ? La confidentialité des réponses peut-elle être garantie?

- En expliquant comment les données seront utilisées, les chercheurs devraient faire preuve de clarté et de transparence avec les répondants et leurs tuteurs. Les résultats augmenteront-ils la stigmatisation des répondants? Comment et où les données seront-elles stockées?

- Pour le partage des données et des résultats de recherche, les chercheurs devraient clarifier la manière dont les résultats seront utilisés. Les chercheurs devraient avoir un plan clair pour partager les résultats avec les participants avant leur diffusion plus large. Les recherches seront-elles utiles pour les TJA impliqués? Comment la confidentialité et l'anonymat des participants seront-ils assurés? 
seront embauchées pour les projets traitant de sujets sensibles. La formation devrait inclure des exercices pour aider les chercheurs à examiner leurs propres attitudes et croyances concernant les sujets. Les chercheurs doivent être formés à écouter soigneusement les informations reçues des répondants sans faire preuve de jugement. Ils devraient avoir conscience des effets que les questions peuvent avoir sur les répondants et connaître la meilleure manière de réagir, en fonction des réactions des répondants. La formation des enquêteurs devrait inclure des séances pratiques sur la manière d'identifier et de répondre de manière appropriée aux signes d'inconfort, ainsi que la manière de suspendre ou de mettre fin à l'entretien si l'impact des questions devient négatif. Il est recommandé d'adopter une approche centrée sur les TJA, permettant au processus de se dérouler au rythme des TJA et sous leur contrôle (Mudaly et Goddard 2009). Les lois locales sur les conditions et les processus pour déclarer la maltraitance d'enfants doivent également être codifiées pour l'équipe de recherche et respectées pendant le processus de recherche.

En explorant des sujets sensibles avec les TJA, il peut être utile de faire un bilan avec le répondant à la fin de l'activité de collecte des données (Black et Ponirakis, 2000; Carroll-Lind et al. 2006). Les chercheurs devraient être formés à repérer les signes pouvant indiquer que le répondant a besoin d'une intervention clinique, en reconnaissant que certaines questions sensibles ont été couvertes et en fournissant des informations sur la manière d'obtenir un soutien professionnel (Black et Ponirakis 2000). Le répondant devrait être informé de la manière d'obtenir un soutien confidentiel à l'échelle locale (Carroll-Lind et al. 2006).

Enfin, même si des termes tels que «violence» et «exploitation» peuvent être utilisés dans les propositions de recherche, il convient de faire preuve d'une prudence extrême pendant le travail sur le terrain, de manière à ne pas cataloguer la vie ou les expériences des répondants, ce qui pourrait créer de la stigmatisation ou la renforcer. Un effort concerté est conseillé pour s'assurer que les recherches ne soient pas présentées à la communauté comme «l'étude sur la violence» ou en d'autres termes défavorables, et que les membres de la communauté ne s'y réfèrent pas en ces termes.

\subsection{Méthodes et outils de recherche}

Les conseils donnés pour les recherches auprès des mineurs veillent non seulement à protéger les enfants, mais également leur capacité à participer réellement et à comprendre pleinement les questions posées. L'objectif des recherches avec le groupe d'âge des TJA est normalement de révéler les expériences et les avis des répondants d'une manière sûre et constructive pour eux. Les outils devraient, dans l'idéal, être centrés sur les TJA pour encourager leur participation active et leur permettre de se faire entendre.

Les recherches auprès des TJA relèvent souvent de deux catégories générales: (1) quelle est la prévalence d'une caractéristique particulière ou d'un résultat particulier, et (2) quels sont les processus ou les explications soulignant comment et pourquoi divers phénomènes se produisent. La première catégorie nécessite de larges échantillons représentatifs et un outil de diagnostic rapide, généralement une enquête structurée. La deuxième catégorie nécessite une exploration en profondeur avec un petit nombre de types de groupes ou d'individus sélectionnés de manière systématique, en utilisant des outils moins structurés.

\section{Des outils quantitatifs structurés pour établir la prévalence}

Cartographie de recensement de la communauté où résident les TJA-les répondants sont des membres adultes $d u$ ménage ou les tuteurs

Un recensement est utilisé pour établir où et combien de personnes par âge et par sexe résident dans une zone géographique définie. Les technologies de système d'information global (SIG) nous permettent de produire rapidement des cartes spatiales précises qui représentent ces données. Un exemple d'application récente de la cartographie de recensement de la communauté axée sur la population des TJA est l'outil « Girl Roster » utilisé pour planifier et cibler les programmes (Bruce et David 2015). Avec cet outil, un entretien de 5 à 10 minutes est effectué dans chaque ménage de la communauté. Les réponses, ainsi que 
les données GPS de l'emplacement du ménage, sont enregistrées sur un agenda électronique, puis téléchargées en temps réel sur un serveur central. Les questions à propos des TJA résidents peuvent comprendre: le sexe, l'âge, la présence des parents, la scolarisation, le fait de savoir si la classe qu'ils suivent correspond à leur âge, leur situation matrimoniale, la parité et les moyens de subsistance. Cette approche détaillée est efficace pour situer les TJA sur une échelle de vulnérabilité au niveau local, en fonction du soutien social, économique et parental. Le contenu des programmes, ainsi que le recrutement, dans la communauté peuvent être guidés par ces informations disponibles rapidement.

Pour mieux visualiser la diversité des jeunes adolescents et leurs besoins, les données d'EDS peuvent également être organisées en cartes spécifiques pour chaque pays (de nombreuses cartes sont disponibles dans la série de guides «Adolescent-Experience In-Depth» publiés par le Population Council). Cartographier les variations des résultats des adolescents dans les régions à l'échelle infranationale peut permettre de souligner les zones géographiques présentant des obstacles pour la santé ou caractérisées par de fortes concentrations d'effets négatifs pour les TJA. Comme le montre la Figure 7, la prévalence des filles mariées avant l'âge de 15 ans a atteint $42 \%$ dans la région Amhara en Éthiopie, ce qui dépasse de beaucoup le taux des autres régions. Une analyse infranationale permet également d'identifier des zones de vulnérabilité composite (voir la Figure 8). Visualiser les données actuelles en utilisant des cartes peut permettre de mieux informer le développement des programmes et des politiques en identifiant les zones d'intervention prioritaires, qui pourraient autrement être masquées par les moyennes nationales.

\section{L'utilisation des services par les TJA- L'enquête auprès des prestataires}

Un autre outil utile dans les études axées sur les TJA est l'analyse des informations existantes et/ou la collecte rapide d'informations sur les personnes qui utilisent différents types de services au sein de la communauté. Les centres de jeunesse, les cliniques et les autres prestataires de services enregistrent fréquemment des infor-
FIGURE 7 Pourcentage de femmes âgées de 20 à 24 ans qui étaient mariées avant l'âge de 15 ans, Éthiopie, 2005

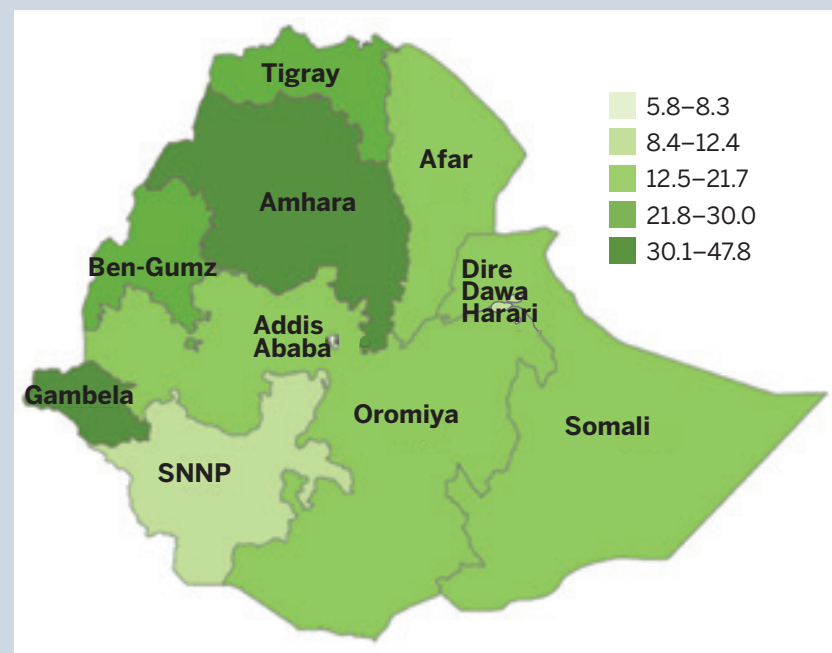

Source: Population Council. 2015b. “The Adolescent Experience In-Depth: Using Data to Identify and Reach the Most Vulnerable Young People: Ethiopia, 2005." New York: Population Council.

FIGURE 8 Pourcentage de filles âgées de 10 à 14 ans non scolarisées et ne vivant avec aucun de leurs parents, Guatemala, 2002/2006

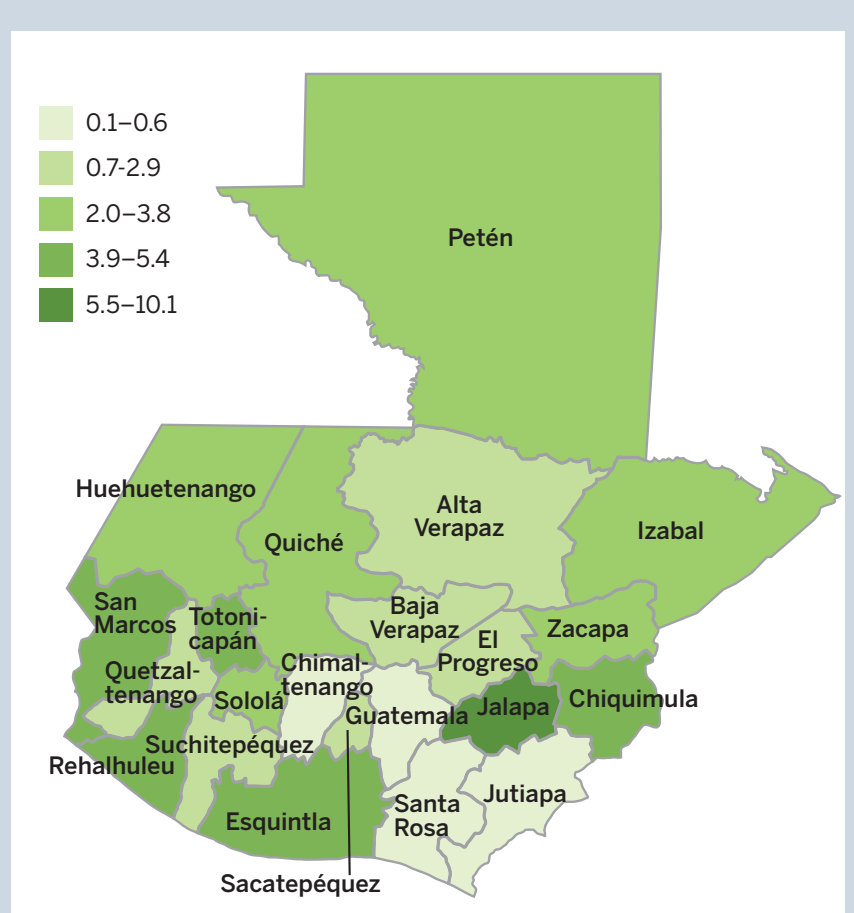

Source: Population Council. 2015b. "The Adolescent Experience InDepth: Using Data to Identify and Reach the Most Vulnerable Young People: Guatemala 2002/2006." New York: Population Council. 
ENCADRÉ 4 Des entretiens en face à face ou assistés par ordinateur?

La question de réaliser des enquêtes avec des adolescents en utilisant des méthodes d'entretien en face à face (EFAF) ou d'auto-entretien audio assisté par ordinateur (ACASI) est difficile à traiter. Le coût, la faisabilité et l'acceptabilité dans le contexte de l'étude sont des questions clés à évaluer pour faire ce choix. Un examen systématique et une méta-analyse des outils d'entretien quantitatifs pour étudier les comportements auto-déclarés associés auVIH et aux IST dans les pays à revenu faible et moyen (Phillips et al. 2010) ont conclu que les différences entre l'EFAF et les auto-entretiens pour les comportements sensibles n'étaient pas uniformes, et qu'aucune méthode n'était nettement meilleure que l'autre. L'examen a également observé des variations dans le niveau des comportements de déclaration en fonction du résultat, de l'étude et des caractéristiques de la population. En résumé, les entretiens en face à face ne sont pas toujours inférieurs aux autres outils d'entretien, en fonction de la sensibilité de la question et de la population évaluée.

mations démographiques de base concernant les utilisateurs de leurs services. Pour évaluer si les TJA se servent des services, les chercheurs peuvent travailler avec les prestataires pour compiler un profil des caractéristiques démographiques des utilisateurs au cours du mois précédent. Dans le cas où ces informations ne sont pas déjà recueillies, un outil simple, l'exercice de couverture des programmes (Program Coverage Exercise) est disponible (Austrian et Ghati 2010) pour permettre aux prestataires de services d'enregistrer des données anonymes à mesure que les utilisateurs viennent pour obtenir des services; celles-ci sont généralement enregistrées pendant une période d'un mois, mais la durée est flexible.

Lorsque cet outil est associé à une cartographie de recensement des ménages, il est possible d'établir une comparaison entre les TJA de la communauté et les types d'adolescents qui utilisent réellement les services (Lardoux et N'Bouke
2013). Des efforts peuvent ensuite être déployés en vue de cibler ceux qui n'accèdent pas aux ressources de la communauté et d'affiner les offres de services pour mieux répondre à ceux qui sont laissés de côté.

\section{Enquêtes basées sur les ménages-étudier les TJA}

Bien qu'il soit possible d'obtenir des informations sur des sujets moins controversés concernant les TJA, comme l'éducation, la santé et les modes de vie auprès de leurs tuteurs, il n'existe toujours pas de méthode fiable convenue pour obtenir, dans un large échantillon, des mesures de prévalence sur des sujets plus sensibles touchant les adolescents, comme la maltraitance et les expériences sexuelles. II est nécessaire d'obtenir davantage de preuves pour déterminer si les TJA de différents âges, sexes et niveaux d'éducation disposent de la capacité cognitive, émotionnelle et de la maturité nécessaires pour comprendre et répondre aux questions d'enquête nécessitant une évaluation réfléchie des obstacles qu'ils rencontrent ou des conséquences potentielles de leurs actions. Cependant, il est important de poursuivre les efforts visant à affiner les outils, car les données à l'échelle mondiale (EDS, VACS, etc.) indiquent qu'un pourcentage important d'adolescents (en particulier de filles) auront une expérience sexuelle et/ou de violence avant l'âge de 15 ans. Le fait qu'il existe un précédent récent (dans les enquêtes VACS, etc.) pour l'inclusion des 13-14 ans dans les enquêtes à grande échelle qui évaluent ces résultats est encourageant.

Si l'on considère que les TJA dans un contexte particulier ont la capacité de traiter ce type de questions, une préoccupation importante dans le domaine est que la peur et/ou la stigmatisation peuvent causer une sous-déclaration des comportements et expériences sensibles par les TJA qui seront ensuite sous-estimés dans la population. II n'existe aucune preuve systématique sur cette question. Cette préoccupation peut être atténuée en demandant à un groupe légèrement plus âgé d'aborder les expériences qu'ils ont connues Iorsqu'ils étaient des TJA. (Voir l'Encadré 4 sur les techniques d'entretien.) II est également utile 
de recueillir des données sur les précurseurs des comportements, car certains résultats d'intérêt (comme les comportements sexuels) peuvent être relativement peu courants au sein de certaines populations de TJA.

\section{Les outils participatifs pour appréhender les processus et explications du comment et du pourquoi}

Pour tous les outils participatifs, il est idéal que les participants soient systématiquement sélectionnés en fonction de critères clairs pertinents pour l'étude (l'âge, le genre, le statut socio-économique, la situation matrimoniale, la survie des parents, la résidence des parents, la scolarisation, etc.); sinon, un petit nombre de participants peuvent produire des résultats non représentatifs. Les animateurs et les enquêteurs devraient avoir l'expérience des méthodes de recherche axées sur les TJA et y avoir été formés, en faisant attention au rythme, au niveau d'énergie, à la capacité d'attention et à la gêne possible du répondant, et savoir comment y réagir, par exemple en accordant des pauses aux TJA et/ou en mettant fin à la séance de groupe ou à l'entretien lorsque le répondant semble éprouver de l'angoisse.

Choix des méthodes qualitatives participatives avec les groupes de TJA

Pour toutes les méthodes participatives en groupe avec les TJA, nous recommandons fortement de diviser les groupes en fonction de l'âge et du genre, avec les 10-12 ans et les 13-14 ans dans des groupes séparés. Les suggestions suivantes peuvent être adaptées en fonction du thème et du contexte des recherches:

- Les groupes de discussion-un événement organisé pendant lequel un chercheur sélectionne et rassemble un groupe de personnes pour discuter et commenter, à partir de l'expérience personnelle, le thème sur lequel porte la recherche.

- La cartographie communautaire participative-dans le contexte d'un groupe de discussion, les répondants utilisent du papier et des marqueurs pour représenter les frontières et les caractéristiques de leur communauté; diverses questions peuvent être explorées, comme la sécurité, l'accès aux espaces publics, l'accès aux ressources, etc.

- Le classement-cette technique produit une liste ordonnée de préférences des TJA ou de raisons concernant un domaine particulier, par exemple, les raisons de ne pas aller à l'école, classées de la plus importante à la moins importante.

- Le changement le plus important-la procédure implique la collecte, la sélection systématique et l'analyse des histoires locales concernant les changements importants perçus, entraînés par les projets de développement; cette approche peut être appropriée uniquement avec les TJA de 13 à 14 ans.

Choix des méthodes participatives avec des TJA en tant qu'individus

- Les entretiens approfondis-il s'agit d'une courte liste de questions utilisée pour explorer un sujet particulier avec une personne; la technique devrait encourager les répondants à développer des thèmes subjectifs en lien avec leurs propres histoires; cette approche peut être appropriée uniquement avec les TJA de 13 à 14 ans.

- Écrire un journal-des notes quotidiennes guidées sur certains thèmes permettent auxTJA de partager les pensées qui peuvent ne pas apparaître dans les contextes d'entretiens en face à face ou en groupe; l'alphabétisation est nécessaire, et la technique peut s'avérer plus fructueuse avec les TJA de 13-14 ans.

Il existe un certain nombre de ressources et d'outils qui fournissent des conseils sur ces techniques et d'autres, y compris quelles méthodes utiliser pour des sujets de recherche et groupes d'âge particuliers, et des instructions par étapes sur la manière de les appliquer. Schenk et Williamson (2005) présentent une liste complète de ressources dans l'Annexe 1 de leur rapport sur les conseils en matière d'éthique. Aitken et Herman (2009), et Ager, Akesson et Schunk (2010) fournissent des examens d'outils participatifs axés sur les enfants. 
Voici quelques séries d'outils produites entre2005 et 2015 :

- Adolescent Girls' Programming Toolkits (Population Council 2015)

- Participatory Tools for Evaluating Psychosocial Work with Children in Areas of Armed Conflict (Hart et al. 2007)

- Conducting a Participatory Situation Analysis of Orphans and Vulnerable Children Affected by HIVI AIDS: Guidelines and Tools (DeMarco 2005)

- Kit of Tools for Participatory Research and Evaluation with Children, Young People and Adults: A compilation of tools used during a Thematic EvaIuation and Documentation on Children's Participation in Armed Conflict, Post Conflict and Peace Building, 2006-2008. (Save the Children 2008)

Les méthodes mixtes : évaluer la prévalence tout en obtenant des informations nuancées

L'approche des méthodes mixtes est prometteuse pour les recherches avec les TJA. De nombreuses définitions des méthodes mixtes sont disponibles. Les thèmes courants dans l'approche de recherche utilisant des méthodes mixtes sont (Creswell et Clark 2007) :

- Un accent sur les questions de recherche qui améliorent la compréhension contextuelle dans la vie réelle, les perspectives à plusieurs niveaux et les influences culturelles

- L'utilisation d'outils quantitatifs rigoureux pour évaluer l'ampleur et la fréquence des notions, et des outils qualitatifs rigoureux pour explorer la signification et la compréhension de ces notions

- L'utilisation de méthodes multiples (par ex., les essais randomisés contrôlés et les entretiens approfondis)

- Une association intentionnelle des méthodes pour tirer parti des atouts de chacune
Les enquêtes utilisant des méthodes mixtes comprennent diverses sources et de nombreux niveaux qui influencent un problème donné (par ex., les politiques, les organisations, la famille, les personnes). Les méthodes quantitatives (principalement déductives) sont idéales pour mesurer la prévalence des phénomènes «connus». Les méthodes qualitatives (principalement inductives) permettent d'identifier des processus précédemment inconnus, des éléments expliquant pourquoi et comment les phénomènes se produisent, et leurs divers effets (Pasick et al. 2009). La recherche utilisant des méthodes mixtes nécessite plus que la simple collecte de données qualitatives pendant les entretiens, ou la collecte de plusieurs formes de preuves qualitatives (par ex., observations et entretiens) ou de plusieurs types de preuves quantitatives (par ex., les enquêtes). Elle implique la collecte intentionnelle de données quantitatives et qualitatives, et associe les atouts de chaque méthode pour répondre aux questions de recherche. 



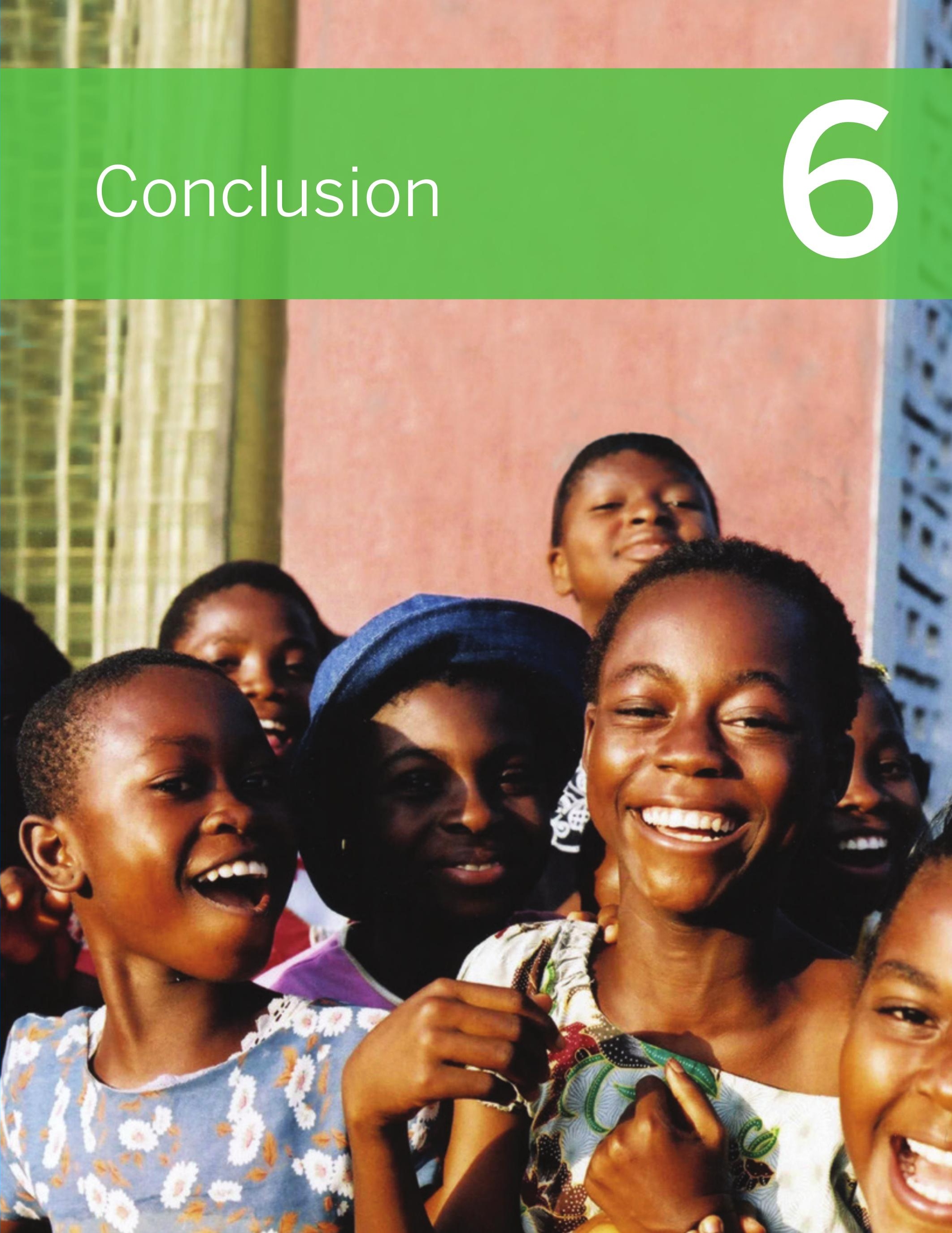


La nature et la qualité de l'avenir des très jeunes adolescents dépendent de la manière dont ils parviennent à négocier la transition vers les rôles de citoyens, d'époux, de parents et de travailleurs. Bien que nous ayons maintenant une image plus claire de la vie des TJA qu'avant, et une confirmation du besoin d'intervenir tôt, la pénurie de recherches sur ce groupe d'âge persiste et entraîne des lacunes de connaissance sur les facteurs essentiels qui définissent les contours de la vie des jeunes. Les chercheurs et les planificateurs de programmes doivent reconnaître que de nombreuses transitions clés dans la vie des jeunes adolescents ne se jouent probablement pas sur un intervalle de cinq ans, mais sur des périodes beaucoup plus courtes: des intervalles d'un, deux ou trois ans. À la lumière des transitions rapides du début de l'adolescence, nous recommandons de décomposer toute analyse future des données en ces deux ou trois périodes plutôt qu'en utilisant le groupe d'âge habituel de cinq ans, car de telles analyses tendent à homogénéiser des expériences extrêmement diverses. Ce faisant, une image plus précise des périodes de seuil pour le changement apparaîtra à mesure que les recherches progressent.

\subsection{Les interventions programmatiques à tester}

Pour établir un plan de programmation, plusieurs sujets mis en évidence dans cet examen méritent davantage d'attention, d'expérimentation et d'évaluation. Au sein de chaque catégorie générale d'interventions potentielles, une myriade de questions de recherche pourraient être posées. La prochaine étape importante serait d'identifier les questions clés et d'élaborer des outils adaptés à l'âge, au genre et aux capacités cognitives pour y répondre.

\section{APPEL À L'ACTION POUR AMÉLIORER LES RECHERCHES}

Pour plaider en faveur d'une attention plus importante accordée aux TJA, nous devons affirmer la nécessité d'obtenir davantage de données de meilleure qualité pour éclairer la situation unique et la diversité des circonstances qui influencent la vie des jeunes garçons et filles. Grâce à une collecte systématique de données issues de sources diverses, nous pouvons décrire de manière plus complète leur vie.

\section{Les mesures suivantes sont nécessaires:}

- Valider des méthodes de collecte d'information, différencier les outils et techniques qui fonctionnent pour les filles de ceux qui fonctionnent pour les garçons, et pour les jeunes par rapport aux TJA plus âgés.

- Établir un dépôt de données où il est possible d'accéder aux enquêtes et autres informations sur les TJA.

- Effectuer de nouvelles analyses des données existantes, ventilées par âge et par genre, pour obtenir des informations sur des sujets plus variés.

- Ventiler les données en intervalles de 2 et3ans (par ex., 10-12 ans; 13-14 ans).

- Se concentrer sur les populations de TJA marginalisées, y compris les jeunes filles mariées, les garçons et les filles vivant dans des bidonvilles urbains, les migrants, les réfugiés et d'autres populations vulnérables.

- Intégrer les recherches aux initiatives de programmation.

- Établir la base de preuves pour les programmes selon ce qui fonctionne, pour quels segments de la population des TJA et dans quelles circonstances.

- Effectuer des recherches à la fois pour guider le processus d'expansion et pour mesurer l'impact de ces efforts. 


\section{Sensibilisation à la santé, promotion de la santé et prévention des maladies}

Créer une culture de la santé et promouvoir le concept du «droit à la santé» pourrait permettre de jeter les bases de futurs plus sains pour beaucoup de TJA dans le monde. Comme cela a été noté ailleurs, les jeunes adolescents répondent davantage aux récompenses qu'aux punitions; les approches positives et favorisant l'autonomisation sont donc susceptibles d'être une stratégie plus efficace, présentant potentiellement des avantages à long terme. Exemples d'activités de promotion de la santé qui mériteraient d'être expérimentées:

- l'« examen de santé et du bien-être (y compris la santé mentale)

- l'éducation complète à la sexualité

- les sports et l'éducation physique

- les jardins communautaires, l'éducation à la nutrition et le choix d'aliments sains

- l'éducation à la puberté

Ces tactiques et d'autres peuvent contribuer à créer une «demande » d'informations, de services et de programmes liés à la santé qui auront des bénéfices à plus long terme.

Étant donné l'émergence des maladies non transmissibles (MNT) en tant que facteur clé de la morbidité et de la mortalité à l'échelle mondiale, il est nécessaire d'accorder une attention plus explicite à la compréhension de leurs fondements sociaux et comportementaux. Il est également nécessaire de sensibiliser à l'importance de traiter l'émergence des problèmes de santé mentale et des comportements qui contribuent à une mauvaise santé plus tard au cours de la vie. Ce champ d'intervention pourrait constituer un domaine important et opportun de programmation, car les PRFM commencent à être aux prises avec de nouveaux défis et opportunités de santé, pour influencer la trajectoire de santé de cette génération de jeunes.

\section{L'éducation complète à la sexualité}

La nécessite d'une ECS pour les adolescents est claire. Quel que soit leur âge, leur genre ou le lieu où ils vivent, les jeunes adolescents sont mal informés à propos de leur corps, de leur santé et de leurs droits. L'ECS peut être assurée par le biais des écoles dans le secteur de l'éducation, ou divers types de plateformes communautaires. II serait utile de tester différentes approches d'ECS dans divers contextes. Un examen complet des programmes axés sur les compétences de la vie courante permettrait également de clarifier quelles compétences sont les plus efficaces pour des domaines particuliers de la santé et du bien-être.

\section{Les programmes liés à la puberté}

Étant donné l'importance de la puberté pour les TJA, il pourrait être utile d'expérimenter des programmes liés à la puberté. Le contenu d'un «programme sur la puberté» devrait nécessairement varier pour s'adapter au contexte et au genre, et inclurait l'ECS comme décrit ci-dessus. Pour les filles, une attention explicite à, et peutêtre également de nouveaux outils sur, l'hygiène menstruelle et la gestion des menstruations pourraient être envisagés. La conscience du corps et l'estime du corps sont importantes, en particulier pour les filles. II pourrait également être utile d'investir dans l'identification des technologies de santé clés pour ce groupe d'âge (par ex., le vaccin contre le VPH) et les plateformes permettant une fourniture sûre et appropriée des produits de santé émergents. Différents concepts et contenus d'une «programmation pour la puberté » pourraient être élaborés et testés. En parallèle, il est nécessaire d'effectuer des recherches de base pour en savoir plus sur le moment et la nature de la transition vers la puberté et ses conséquences pour la mobilité, la scolarité, le mariage, les normes de genre, etc.

\section{Normes de genre, équité et droits}

Les programmes spécifiquement axés sur les normes de genre et les comportements peuvent apporter de nombreux avantages. Les travaux dans ce domaine sont naissants mais prometteurs. L'ECS est un exemple de programme qui vise à promouvoir l'égalité des sexes, mais il en existe d'autres. La plupart des programmes visant à réduire le mariage des enfants et d'autres pratiques traditionnelles néfastes se concentrent fondamentalement sur les normes de genre et sur le fait de garantir les droits des filles. Un autre domaine important à explorer est l'accès des filles 
aux espaces et ressources communautaires, au droit de jouer, etc. II est nécessaire de concevoir, de mettre en œuvre et d'évaluer des programmes plus nombreux et variés visant à faire évoluer les normes de genre, afin d'établir une base de preuves sur ce sujet.

\section{Les transitions éducatives}

Jusqu'à présent, une quantité considérable de travaux ont été effectués autour des programmes de transfert monétaire. II vaudrait la peine de continuer à expérimenter ces programmes, en particulier les incitations qui encouragent la transition de l'école primaire à l'école secondaire (par ex., des primes d'incitations plus importantes au début du secondaire). II serait également utile d'étudier si des incitations versées aux enseignants ou des incitations versées aux étudiants en fonction de leurs résultats scolaires permettraient d'améliorer les progrès scolaires dans le secondaire. Un secteur émergent essentiel pour l'expérimentation serait des initiatives de sécurité scolaire conçues de manière rigoureuse. Enfin, des innovations visant à améliorer le contenu de l'éducation, la qualité et la pédagogie sont nécessaires, y compris l'expérimentation de nouvelles approches, l'enseignement de compétences de pensée critique, l'éducation complète à la sexualité au sein des écoles et la compréhension de l'impact des TIC.

\section{Les interventions parentales}

Malgré la promesse des interventions parentales pour les populations des PRFM, il existe un manque évident d'études de haute qualité sur les interventions axées sur les compétences parentales en général, et en particulier auprès des très jeunes adolescents. II est nécessaire de trouver des études de programmes de compétences parentales dont la taille d'échantillon est suffisante, qui possèdent une conception rigoureuse (expérimentale ou quasi-expérimentale), l'évaluation des effets sur le comportement des parents et des enfants, et la mesure des comportements, non seulement par l'auto-déclaration mais aussi par des observations directes des interactions parents-enfants. Les interventions parentales pour lutter contre les mauvais traitements peuvent per- mettre de réduire la violence dont sont victimes les TJA, et le risque qu'ils commettent eux-mêmes ces violences plus tard.

\subsection{Regarder vers l'avenir}

Nous avons passé en revue plusieurs domaines clés qui pourraient bénéficier de plus d'expérimentation et de nouvelles recherches. Le fait de démarrer des programmes autour de cet âge est une manière de reconnaître l'évolution des capacités des jeunes et leurs besoins distincts. En recueillant des données sur les risques et les opportunités qui se présentent aux TJA filles et garçons, il est possible de développer des stratégies pour les cibler de manière efficace, en leur fournissant un mélange adapté d'informations, de compétences et de services. Le choix de moments stratégiques pour mettre en œuvre les interventions permet d'obtenir des résultats positifs avant que l'architecture de la vie des jeunes adolescents ne soit déterminée.

Étant donné l'immense diversité de la population des très jeunes adolescents, aucune méthodologie ou approche ne pourrait fonctionner seule dans tous les milieux ou avec tous les sousgroupes de TJA. Nous devons en apprendre davantage sur le moment, la nature et les conséquences des transitions clés que traversent les jeunes adolescentes et adolescents, et en particulier comment celles-ci se déroulent chez les groupes les plus vulnérables.

Alors que nous regardons vers l'avenir, nous envisageons une stratégie de recherche et d'action plus forte, plus audacieuse et plus dynamique pour soutenir les politiques et programmes innovants qui offriront aux adolescentes et aux adolescents une transition sûre, saine et productive vers l'âge adulte. Le pont qui relie l'enfance et le début de l'âge adulte est beaucoup trop précaire pour de nombreux enfants du monde en développement. Le renforcement de ce passage pour s'assurer qu'il conduise vers un avenir plus radieux mérite que nous y consacrions davantage d'attention. Nous sommes convaincus que c'est le moment de mettre en œuvre une nouvelle génération d'interventions de recherche et de programmes consacrés aux très jeunes adolescents. 


\section{Postface}

\section{Le moment est venu}

Ce rapport Investir Quand Cela Compte vient appuyer les arguments et les preuves énoncés dans le rapport de 2006. Même si la période de 10-14 ans est censée être un moment paisible où les filles et les garçons vont à l'école, grandissent en bonne santé et acquièrent les compétences nécessaires pour passer avec confiance de l'enfance au début de l'âge adulte, ce processus de «transition » idéalisé par étapes s'avère rarement vrai pour les filles et les garçons les plus pauvres et exclus, dans les communautés les plus défavorisées du monde.

Dans de nombreux milieux, la puberté est une expérience négative pour les filles, qui apporte davantage de restrictions et de doutes. Les filles perçoivent les restrictions spatiales qui s'appliquent à leurs mouvements comme une exclusion des opportunités offertes dans la vie (Hallman et al. 2014). Les filles sont soumises à de nombreuses violations graves des droits humains, souvent permanentes, comme les mutilations génitales féminines et la contrainte sexuelle qui laissent une trace indélébile pouvant entraver l'émergence des actions, et pouvant entraîner un mariage et une grossesse alors que les filles sont encore des enfants ( «le mariage d'enfants », et non le mariage précoce), et le fait de contracter des maladies sexuellement transmissibles lors de rapports forcés avec des partenaires plus âgés. Et bien qu'elles ne soient pas préparées à ce rôle, les adolescentes dans de nombreuses régions du monde peuvent s'attendre à devoir subvenir à leurs besoins et à ceux de leurs enfants seules ou en grande partie, étant donné les taux extrêmement élevés et stables de monoparentalité causés par le divorce, le veuvage et l'abandon économique. Pendant la période de 10 à 14 ans, les adolescents les plus stressés, en particulier les filles, peuvent migrer ou être forcés à migrer pour quitter les fortes pressions de leur lieu natal et le manque d'opportunités, et aller vers une situation qui peut être tout aussi difficile et vers des possibilités inconnues dans les milieux urbains.

Et pourtant, il n'y a quasiment pas de politiques et de programmes consacrés aux10-14 ans, à part le projet trop optimiste axé sur leur présence à l'école. La scolarité seule, en particulier compte tenu de la qualité généralement faible des écoles dans les communautés les plus pauvres, ne peut pas porter le poids des besoins des jeunes adolescents en cette période de croissance et de défis immenses. D'autres compétences, en dehors des compétences cognitives, doivent être explicitement prises en compte: l'éducation financière, l'établissement d'habitudes saines et d'épargne, la documentation personnelle, la connaissance des droits et des moyens de les faire valoir, et une citoyenneté active. Il s'agit d'une période charnière essentielle et décisive, en particulier pour les filles. En l'absence d'un plan et d'un «pont» pour soutenir le passage à l'âge adulte, la sexualité et la fertilité émergentes des jeunes filles, et le travail des garçons et des filles (leur potentiel de vie, essentiellement), peuvent être appropriés par les familles et les communautés sous le prétexte de la «culture», et intensifiés par le manque.

Sans un ensemble vigilant et responsable de programmes accessibles basés sur la communauté, les filles et les garçons les plus défavorisés des communautés les plus pauvres ne se hisseront pas dans les marchés émergents ou les positions de leadership, mais seront au contraire tirés vers le bas pour subventionner les crises familiales et les chocs de la société. Les jeunes adolescents, en particulier les filles, sont profondément affectés 
par les urgences climatiques (comme les inondations saisonnières, les catastrophes naturelles, les conflits et les maladies infectieuses, qu'il s'agisse des épidémies «lentes» comme le VIH ou rapides comme l'Ebola). La négligence, pendant ce moment vital, crée de la marginalisation et constitue la base de la pauvreté individuelle et collective.

Il est maintenant temps d'accorder la priorité à des droits explicites pour les 10-14ans, en particulier ceux des communautés marginalisées, jusqu'au niveau infranational. Les plans devront être spécifiquement adaptés au genre, car la vie des garçons et celle des filles divergent radicalement, et sont caractérisées par des risques et des opportunités distincts.

Comblons donc ces lacunes. Il existe une pléthore de programmes pour les enfants de moins de cinq ans, et les «droits des adultes» sont théoriquement disponibles à l'âge adulte, à l'âge de la majorité sexuelle ou à l'âge de 18 ans (qui sont généralement les mêmes). Que se passe-t-il entre-temps?

Nous devons formuler et exécuter des programmes pour les très jeunes adolescents qui renforcent leurs atouts de santé, sociaux, économiques et cognitifs. Au cœur de cette question est la création de plateformes communautaires/lieux de rencontre, des endroits permettant un renfor- cement des compétences et un engagement en toute sécurité, en particulier pour les filles dont l'espace social est déjà confiné et qui dans de nombreux cas, se rétrécit. Un accès véritable nécessite des campagnes d'informations pratiques à l'échelle locale (où les TJA obtiennent-ils une pièce d'identité?) et des liens concrets (où les filles obtiennent-elles des bons pour les services?) dans les espaces qu'ils fréquentent quotidiennement. Même si les droits sont valables à l'échelle mondiale, leur accès est local. Si les très jeunes adolescents ne les connaissent pas, ils ne peuvent pas les rechercher. S'ils ne peuvent pas marcher jusqu'à eux, ils ne peuvent pas les obtenir. Même s'ils sont dynamiques à l'échelle nationale, ces programmes pour les TJA doivent être affirmés au niveau infranational et mis en œuvre à l'échelle des communautés pour être efficaces. L'objectif d'une politique explicite pour les jeunes adolescents devrait être d'assurer, par le biais de ressources et de structures locales dédiées, que les TJA soient en sécurité, heureux et productifs peu importe qui ils sont et où ils vivent.

JUDITH BRUCE ASSOCIÉE PRINCIPALE ET ANALYSTE POLITIQUE PROGRAMME PAUVRETÉ, GENRE ET JEUNESSE POPULATION COUNCIL 


\section{Références}

Adukia, Anjali. 2013. "Sanitation and Education." Cambridge, MA: Harvard Graduate School of Education. www.open.uwi.edu/hflecaribbean/ sites/default/files/adukia_sanitation_and_ education.pdf.

Ager, Alastair, Bree Akesson et Kelly Schunk. 2010. "Mapping of child protection monitoring and evaluation tools, Final Report." New York: Columbia University and CPC Learning Network.

Aitken, Stuart et Thomas Herman. 2009. "Literature review on qualitative methods and standards for engaging and studying independent children in the developing world." Florence: UNICEF Innocenti Research Centre.

Amin, Sajeda, Sigma Ainul, Farhana Akter, et al. 2014. "From evidence to action: Results from the 2013 baseline survey for the BALIKA project." New York: Population Council. www.popcouncil.org/uploads/pdfs/2014PGY_ BALIKA_report.pdf.

Andrews, Gavin, Justine Corry, Tim Slade, Cathy Issakidis et Heather Swanston. 2004. "Child sexual abuse," in Comparative Quantification of Health Risks: Global and Regional Burden Of Disease Attributable to Selected Major Risk Factors, pp. 1851-1940.

Austrian, Karen et D. Ghati. 2010. “Girl-centered program design: A toolkit to develop, strengthen, and expand adolescent girls programs." Nairobi: Population Council. www.popcouncil. org/girlcenteredprogram.

Austrian, Karen et Eunice Muthengei. 2013. “Safe and smart savings products for vulnerable adolescent girls in Kenya and Uganda." Evaluation Report. New York: Population Council.

Baird, Sarah, Francisco H.G. Ferreira, Berk Özler et Michael Woolcock. 2014. "Conditional, unconditional and everything in between: A systematic review of the effects of cash transfer programmes on schooling outcomes," Journal of Development Effectiveness 6(1): 1-43.

Baldwin, Susie B., David P. Eisenman, Jennifer N. Sayles, Gery Ryan et Kenneth S. Chuang. 2011. "Identification of human trafficking victims in health care settings," Health and Human Rights 13(1): E36-49.

Banerjee, Abhijit, Paul Glewwe, Shawn Powers et Melanie Wasserman. 2013. "Expanding access and increasing student learning in post-primary education in developing countries: A review of the evidence." Cambridge: Massachusetts Institute of Technology. www.povertyactionlab. org/sites/default/files/publications/PPE\%20 Review\%20Paper\%20April\%202013.pdf.

Bankole, Akinrinola, Ann Biddlecom, Georges Guiella, Susheela Singh et Eliya Zulu. 2007. "Sexual behavior, knowledge and information sources of very young adolescents in four sub-Saharan African countries," African Journal of Reproductive Health 11(3): 28-43.

Baptiste, Donna R., Chisina Kapungu, Steve Miller, Laurel Crown, David Henry, Dona Da Costa Martinez et Karen Jo-Bennett. 2009. “Increasing parent involvement in youth HIV prevention: A randomized Caribbean study," AIDS Education and Prevention 21(6): 495-511.

Barker, Gary Thomas. 2000. “What about boys? A literature review on the health and development of adolescent boys." Geneva: World Health Organization.

Barker, Gary Thomas, Christine Ricardo, Marcos Nascimento, et al. 2007. “Engaging men and boys in changing gender-based inequity in health: Evidence from programme interventions. Geneva: World Health Organization. http://asset. atlasproject.eu/who/t_file/664c72a1-9e604fa2-bec9-035459fd46a7/9af80260-bf71-11e1afa7-0800200c9a66/Engaging_men_boys.pdf.

Barrera-Osorio, Felipe et Leigh L. Linden. 2009. “The use and misuse of computers in education: Evidence from a randomized controlled trial of a language arts program." Cambridge, MA: Abdul Latif Jameel Poverty Action Lab (JPAL). www.leighlinden.com/Barrera-Linden\%20 Computadores_2009-03-25.pdf.

Barry, Margaret M., Aleisha M. Clarke, Rachel Jenkins et Vikram Patel. 2013. "A systematic review of the effectiveness of mental health promotion interventions for young people in low and middle income countries," BMC Public Health 13: 835. doi:10.1186/1471-2458-13-835.

Beaman, Lori, Esther Duflo, Rohini Pande et Petia Topalova. 2012. "Female leadership raises aspirations and educational attainment for girls: A policy experiment in India," Science 335 (6068): 582-86.

Belfer, Myron L. 2008. "Child and adolescent mental disorders: The magnitude of the problem across the globe," Journal of Child Psychology and Psychiatry, and Allied Disciplines 49(3): 226-236. doi:10.1111/j.1469-7610.2007.01855.x. 
Bellis, M.A., J. Downing et J.R. Ashton. 2006. “Adults at 12 ? Trends in puberty and their public health consequences," Journal of Epidemiology and Community Health 60(11): 910-911.

Bhabha, Jacqueline. 2008. "Independent children, inconsistent adults: International child migration and the legal framework." Florence: UNICEF Innocenti Research Centre. ideas.repec.org/p/ ucf/indipa/indipa08-3.html.

Birdthistle, Isolde, Kelly Dickson, Matthew Freeman et Leila Javidi. 2011. "What impact does the provision of separate toilets for girls at schools have on their primary and secondary school enrolment, attendance and completion?: A systematic review of the evidence." London, UK: EPPI-Centre, Social Science Research Unit, University of London.

Black, M. et A. Ponirakis. 2000. "Computer-administered interviews with children about maltreatment methodological, developmental and ethical issues," Journal of Interpersonal Violence 15(7): 682-695.

Black, Robert E., Cesar G. Victora, Susan P. Walker, et al. 2013. "Maternal and child undernutrition and overweight in low-income and middle-income countries," The Lancet 382(9890): 427-451.

Blakemore, Sarah-Jayne. 2008. "The social brain in adolescence," Nature Reviews. Neuroscience 9(4): 267-277. doi:10.1038/nrn2353.

Blakemore, Sarah-Jayne et Kathryn L. Mills. 2014. "Is adolescence a sensitive period for sociocultural processing?" Annual Review of Psychology 65(1): 187-207. doi:10.1146/ annurev-psych-010213-115202.

Blanc, Ann, William Winfrey et John Ross. 2013. New findings for maternal mortality age patterns: Aggregated results for 38 countries. PLoS One 8(4): e59864. http://dx.plos.org/10.1371/journal.pone.0059864.

Blum, Robert, Nan Marie Astone et Michele Decker. 2013. "A conceptual framework for early adolescence: A platform for research and action." Geneva: World Health Organization.

Blum, Robert W., Francisco I.P.M. Bastos, Caroline W. Kabiru et Linh C. Le. 2012. "Adolescent health in the 21st century," The Lancet 379(9826): 1567-68. doi:10.1016/S0140-6736(12)60407-3.

Brady, Martha. 2005. "Creating safe spaces and building social assets for young women in the developing world: A new role for sports," Women's Studies Quarterly 33(1-2) 35-49.

. 2011a. "Leveling the playing field: Building girls' sports programs and creating new opportunities," Promoting Healthy, Safe, and Productive Transitions to Adulthood. Brief No. 1. New York: Population Council. 2011b. "Taking Programs for Vulnerable Adolescents to Scale: Experiences, Insights, and Evidence." Promoting Healthy, Safe, and Productive Transitions to Adulthood. Brief No. 36. New York: Population Council.

Breinbauer, Cecilia et Matilde Maddaleno. 2005. "Promoting healthy behaviors in adolescents," in Youth: Choices and Change, pp. 262-264. Washington, DC: Pan American Health Organization (PAHO). www.paho.org/English/ DD/PUB/Youth.

Brody, Jane. 2015. "Screen addiction is taking a toll on children," The New York Times, July 6, Section: Well. well.blogs.nytimes.com/2015/07/ 06/screen-addiction-is-taking-a-toll-on-children/.

Browne, Evie. 2013. "Child-friendly spaces for adolescent girls in emergency settings." Helpdesk Research Report. Birmingham, UK: GSDRC, University of Birmingham.

Bruce, Judith et Vira David. 2015. "The girls roster toolkit: Seeing and valuing all the girls in your community." New York: Population Council. www.popcouncil.org/uploads/pdfs/Girl_ Roster_flyer.POPCOUNCIL.2015.pdf.

Buckley, Chris et Ellen Barry. 2015. "Rohingya women flee violence only to be sold into marriage," The New York Times, August 2. www.nytimes. com/2015/08/03/world/asia/ rohingya-women-flee-violence-only-to-be-soldinto-marriage.html.

Butchart, A. 2006. "Preventing child maltreatment: A guide to taking action and generating evidence." Geneva: WHO/IPSCAN.

Carroll-Lind, Janis, James W. Chapman, Janet Gregory et Gabrielle Maxwell. 2006. "The key to the gatekeepers: Passive consent and other ethical issues surrounding the rights of children to speak on issues that concern them," Child Abuse \& Neglect 30(9): 979-989. doi:10.1016/ j.chiabu.2005.11.013.

Catino, Jennifer. 2012. "The health of vulnerable adolescent girls: A strategic investment for double return." New York; Population Council et VN Adolescent Girls Task Force.

Caton, Carolyn, Josh Chaffin, Emmanuelle Comping, Mendy Marsh et Jennifer Schulte. 2014. "Economic strengthening to reduce risk of gender-based violence for adolescent girls in humanitarian settings." Washington, DC: Search for Common Ground (SFCG).

CBS News. 2015. "Boko Haram sends girl suicide bombers to attack Nigeria markets." New York: CBS News. www.cbsnews.com/news/bokoharam-girl-suicide-bomber-attack-nigeriamarkets-reports/. 
Centers for Disease Control and Prevention (CDC). 2015. "Physical activity facts." Atlanta: CDC. http://www.cdc.gov/healthyyouth/ physicalactivity/facts.htm.

Child Protection Monitoring and Evaluation Reference Group (CP MERG). 2012. "Ethical principles, dilemmas and risks in collecting data on violence against children: A review of available literature." New York: UNICEF, Statistics and Monitoring Section, Division of Policy and Strategy.

Child Soldiers International (CSI). "Child Soldiers International." 2015. www.child-soldiers.org.

Chong, Erica, Kelly Hallman et Martha Brady. 2006. "Investing when it counts: Generating the evidence base for policies and programmes for very young adolescents." New York: UNFPA et Population Council. www.unicef.org/ InvestingWhenltCounts.pdf.

Chowdhary, Neerja, Siham Sikander, Najia Atif, et al. 2014. "The content and delivery of psychological interventions for perinatal depression by non-specialist health workers in low and middle income countries: A systematic review," Best Practice \& Research Clinical Obstetrics \& Gynaecology 28(1): 113-133.

Clark, Shelley. 2004. "Early marriage and HIV risks in sub-Saharan Africa," Studies in Family Planning 35(3): 149-160.

Clark, Thomas S., Gerhard K. Friedrich, Methembe Ndlovu, Torsten B. Neilands et Willi McFarland. 2006. "An adolescent-targeted HIV prevention project using African professional soccer players as role models and educators in Bulawayo, Zimbabwe," AIDS and Behavior 10(1): 77-83.

Coalition for Adolescent Girls (CAG). 2012. "Missing the emergency: Shifting the paradigm for relief to adolescent girls." www.iywg.org/resources/ missing-emergency-shifting-paradigm-reliefadolescent-girls.

Collin-Vézina, Delphine, Isabelle Daigneault et Martine Hébert. 2013. "Lessons learned from child sexual abuse research: Prevalence, outcomes, and preventive strategies," Child and Adolescent Psychiatry and Mental Health 7(1): 22-30.

Creswell, John W. et Vicki L. Plano Clark. 2007. Designing and Conducting Mixed Methods Research. Thousand Oaks, CA: Sage Publications.

Crone, Eveline A. et Ronald E. Dahl. 2012. “Understanding adolescence as a period of social-affective engagement and goal flexibility," Nature Reviews Neuroscience 13(9): 636-650. doi:10.1038/nrn3313.

Dasgupta, P., K. Tureski, R. Lenzi, K. Bindu et G. Nanda. 2012. "Half the Sky Movement Multi-Media Communication Initiative: An evaluation of the 9-minutes mobile game and video." Washington, DC: D-Change, FHI 360.
Decker, Michele R., Beth Dail Marshall, Mark Emerson, et al. 2014. "Respondent-driven sampling for an adolescent health study in vulnerable urban settings: A multi-country study," Journal of Adolescent Health 55(6): S6-12. doi:10.1016/j. jadohealth.2014.07.021.

DeMarco, Renee. 2005. “Conducting a participatory situation analysis of orphans and vulnerable children affected by HIV/AIDS: Guidelines and tools." Arlington, VA: Family Health International.

Deshpande, Neha A. et Nawal M. Nour. 2013. “Sex trafficking of women and girls," Reviews in Obstetrics and Gynecology 6(1): e22.

Devries, Karen M., Louise Knight, Jennifer C. Child, et al. 2015. "The good school toolkit for reducing physical violence from school staff to primary school students: A cluster-randomised controlled trial in Uganda," The Lancet Global Health 3(7): e378-386.

Dixon-Mueller, Ruth. 2010. “The Sexual and reproductive health of young adolescents in developing countries: Reviewing the evidence, identifying research gaps, and moving the agenda." Report of a WHO Technical Consultation. Geneva: WHO. http://www.who.int/immunization/hpv/target/ the_sexual_and_reproductive_health_of_young adolescents_who_2011.pdf.

Dosenbach, Nico U.F., Binyam Nardos, Alexander L. Cohen, et al. 2010. "Prediction of individual brain maturity using fMRI," Science 329 (5997): 1358-1361. doi:10.1126/science.1194144.

Downing, Jennifer et Mark A. Bellis. 2009. “Early pubertal onset and its relationship with sexual risk taking, early pubertal onset and its relationship with sexual risk taking, substance use and anti-social behaviour: A preliminary cross-sectional study," BMC Public Health 9. doi: 10.1186/14712458-9-446.

Economic Research Forum (ERF). 2006. "Egypt Labor Market Panel Survey (ELMPS) 2006." Egypt: Central Agency for Public Mobilization and Statistics.

Edmonds, Eric et Maheshwor Shrestha. 2009. "Children's work and independent child migration: A critical review." Florence: UNICEF Innocenti Research Centre. ideas.repec.org/p/ucf/inwopa/inwopa586.html.

Erulkar, A., T. Mekbib, N. Simie et T. Gulema. 2004. "The experience of adolescence in rural Amhara Region, Ethiopia." Accra: Population Council.

Erulkar, Annabel et Eunice Muthengi. 2009. “Evaluation of Berhane Hewan: A program to delay child marriage in rural Ethiopia," International Perspectives on Sexual and Reproductive Health 35(1) 6-14.

Eshel, Neir, Bernadette Daelmans, Meena Cabral de Mello et Jose Martines. 2006. "Responsive parenting: Interventions and outcomes," Bulletin of the World Health Organization 84(12): 991-998. 
ExpandNet/WHO Tools. n.d. www.expandnet.net/ tools.htm.

Family Violence Prevention Fund et World Childhood Foundation. 2005. "Turning pain into power: Trafficking survivors' perspectives on early intervention strategies." San Francisco: FVPF. www. futureswithoutviolence.org/turningpain-into-power-trafficking-survivorsperspectives-on-early-intervention-strategies.

Finkelhor, D., S.L. Hamby, R. Ormrod et H. Turner. 2005. "The juvenile victimization questionnaire: Reliability, validity, and national norms," Child Abuse \& Neglect 29: 383-412.

Fulu, Emma, Alice Kerr-Wilson et James Lang. 2014. "What works to prevent violence against women and girls: Evidence Review of Interventions to Prevent Violence against Women and Girls." Pretoria: Medical Research Council. http://r4d.dfid.gov.uk/pdf/outputs/VAWG/ What_Works_Inception_Report_June_ 2014_AnnexF_WG23_paper_prevention_ interventions.pdf.

Giedd, Jay N. 2008. "The teen brain: Insights from neuroimaging," Journal of Adolescent Health 42(4): 335-343. doi:10.1016/j.jadohealth.2008. 01.007.

Gore, Fiona M., Paul J.N. Bloem, George C. Patton, et al. 2011. "Global burden of disease in young people aged 10-24 years: A systematic analysis," The Lancet 377(9783): 2093-2102. doi:10.1016/ S0140-6736(11)60512-6.

Graham, A., M. Powell, N. Taylor, D. Anderson et R. Fitzgerald. 2013. "Ethical research involving children (ERIC)." Florence: UNICEF Innocenti Research Centre.

Grant, Monica J. et Kelly K. Hallman. 2008. "Pregnancy-related school dropout and prior school performance in KwaZulu-Natal, South Africa," Studies in Family Planning 39(4): 369-382.

Grant, Monica J., Cynthia B. Lloyd et Barbara S. Mensch. 2013. "Menstruation and school absenteeism: Evidence from rural Malawi," Comparative Education Review 57(2): 260.

Greenbaum, V. Jordan. 2014. “Commercial sexual exploitation and sex trafficking of children in the United States," Current Problems in Pediatric and Adolescent Health Care 44(9): 245-269.

GSM Association. 2013a. "Children's use of mobile phones: An international comparison." London: GMSA and Japan: NTT DOCOMO.

2013b. "Women and mobile-A global opportunity: A study on the mobile phone gender gap in low and middle-income countries." London: GSMA Development Fund, Cherie Blair Foundation for Women, Vital Wave Consulting.
Guse, Kylene, Deb Levine, Summer Martins, et al. 2012. "Interventions using new digital media to improve adolescent sexual health: A systematic review," Journal of Adolescent Health 51(6): 535-543. doi:10.1016/j.jadohealth.2012.03.014.

Haberland, Nicole A. 2015. "The case for addressing gender and power in sexuality and HIV education: A comprehensive review of evaluation studies," International Perspectives on Sexual and Reproductive Health 41(1): 31-42.

Hadley, Craig, David Lindstrom, Fasil Tessema et Tefara Belachew. 2008. "Gender bias in the food insecurity experience of Ethiopian adolescents," Social Science \& Medicine 66(2): 427-438.

Hallman, Kelly. 2007. "Non-consensual sex, school enrolment and educational outcomes in South Africa," Africa Insight 37(3): 454.

2011. "Social exclusion: The gendering of adolescent HIV risks in KwaZulu-Natal, South Africa," in J. Klot and V. Nguyen (eds.), The Fourth Wave: An Assault on Women-Gender, Culture and HIV/AIDS in the 21st Century, pp. 53-80. New York: Social Science Research Council and Paris: UNESCO.

Hallman, Kelly, K. Govender et Eva Roca. 2007. "Enhancing financial literacy, HIV/AIDS skills, and safe social spaces among vulnerable South African youth." Transitions to Adulthood. Brief 4. New York: Population Council.

. 2015. "Siykaka Nentsha: Local secondary school graduates create safe space classrooms for gendered social, health and financial skills acquisition in Rural South Africa." Unpublished. New York: Population Council.

Hallman, Kelly K., Nora J. Kenworthy, Judith Diers, Nick Swan et Bashi Devnarain. 2014. "The shrinking world of girls at puberty: Violence and gender-divergent access to the public sphere among adolescents in South Africa," Global Public Health 10(3): 279-295.

Hallman, Kelly, Sara Peracca, Jennifer Catino et Marta Julia Ruiz. 2007. "Indigenous girls in Guatemala: Poverty and location," in Maureen A. Lewis et Marlaine E. Lockheed (eds.), Exclusion, Gender and Schooling: Case Studies from the Developing World, pp. 145-175. Washington, DC: Center for Global Development.

Hallman, Kelly et Eva Roca. 2006. "Reducing the Social Exclusion of Girls." Brief No. 27. Promoting Healthy, Safe and Productive Transitions to Adulthood. New York: Population Council.

Halpern, Carolyn Tucker, Ellen M.H. Mitchell, Tilda Farhat et Phil Bardsley. 2008. "Effectiveness of web-based education on Kenyan and Brazilian adolescents' knowledge about HIV/AIDS, abortion law, and emergency contraception: Findings from TeenWeb," Social Science \& Medicine 67(4): 628-37. doi:10.1016/j.socscimed.2008.05.001. 
Han, Chang-Keun, Fred M. Ssewamala et Julia ShuHuah Wang. 2013. "Family economic empowerment and mental health among AIDS-affected children living in AIDS-impacted communities: Evidence from a randomised evaluation in Southwestern Uganda," Journal of Epidemiology and Community Health 67(3): 225-230.

Hart, Jason, Ananda Galappatti, Jo Boyden et Miranda Armstrong. 2007. "Participatory tools for evaluating psychosocial work with children in areas of armed conflict: A pilot in Eastern Sri Lanka," Intervention 5(1): 41-60.

Herman-Giddens, Marcia E. 2006. "Recent data on pubertal milestones in United States children: The secular trend toward earlier development," International Journal of Andrology 29(1): 241-246.

Hermoso, Maria, Vesna Vucic, Christiane Vollhardt, et al. 2011. "The effect of iron on cognitive development and function in infants, children and adolescents: A systematic review," Annals of Nutrition and Metabolism 59 (2-4): 154-165.

Hindin, M.J., P. Bloem et Jane Ferguson. 2015. “Effective nonvaccine interventions to be considered alongside Human Papilloma Virus vaccine delivery." Journal of Adolescent Health 56(1): 10-18.

Hingle, Melanie D., Teresia M. O'Connor, Jayna M. Dave et Tom Baranowski. 2010. "Parental involvement in interventions to improve child dietary intake: A systematic review," Preventive Medicine 51(2): 103-111. doi:10.1016/ j.ypmed.2010.04.014.

Hodge, David R. et Cynthia A. Lietz. 2007. “The international sexual trafficking of women and children: A review of the literature," Affilia 22(2): 163-174.

Idele, Priscilla, Amaya Gillespie, Tyler Porth, et al. 2014. "Epidemiology of HIV and AIDS among adolescents: Current status, inequities, and data gaps," Journal of Acquired Immune Deficiency Syndromes 66 (July): S144-S153. doi:10.1097/ QAI.0000000000000176.

Igras, Susan M., Marjorie Macieira, Elaine Murphy et Rebecka Lundgren. 2014. “Investing in very young adolescents' sexual and reproductive health," Global Public Health 9(5): 555-569. doi:10.1080/17441692.2014.908230.

Institute for Reproductive Health (IRH). 2015a. “' Ending the hesitation around menstruation': GrowUp Smart." Washington, DC: IRH. www.irh.org/blog/ending-hesitation-aroundmenstruation-growup-smart. - 2015b. My Changing Body: Body Literacy \& Fertility Awareness for Young People (2nd Edition). Washington, DC: IRH. http://irh.org/resourcelibrary/my-changing-body-body-literacy-fertilityawareness-for-young-people-2nd-edition/.
Institute for Reproductive Health (IRH) et USAID. 2010. "Reaching Very Young Adolescents (VYAs): Advancing program, research and evaluation practices." Washington, DC: Institute for Reproductive Health, Georgetown University. irh.org/ wp-content/uploads/2013/04/IRH_VYA_ Technical_Consultation_2010_Meeting_ Report_FINAL.pdf.

Institute of Medicine (IOM). 2008. Violence Prevention in Low and Middle-Income Countries: Finding a Place on the Global Agenda. Washington, DC: National Academies Press.

International Society for Prevention of Child Abuse and Neglect (ISPCAN). 2007. World Perspectives on Child Abuse, 6th Edition. Chicago: Aurora.

Jacob, K.S. et Vikram Patel. 2014. "Classification of mental disorders: A global mental health perspective," The Lancet 383(9926): 1433-1435.

Jejeebhoy, Shireen, Iqbal Shah et Shyam Thapa. 2005. Sex Without Consent: Young People in Developing Countries. London: Zed Books.

Jensen, F.E. et A.E. Nutt. 2015. The Teenage Brain: A Neuroscientist's Survival Guide to Raising Adolescents and Young Adults. New York: Harper Collins.

Jensen, Robert. 2010. "The (perceived) returns to education and the demand for schooling," The Quarterly Journal of Economics 125(2): 515-548.

Jimerson, Shane R., Gabrielle E. Anderson et Angela D. Whipple. 2002. "Winning the battle and losing the war: Examining the relation between grade retention and dropping out of high school," Psychology in the Schools 39(4): 441-457.

Johnson, L., Y. Lee, D. Ansong, M. Sherraden, G. Chowa et F. Ssewamala. 2015. "Youth savings patterns and performance in Colombia, Ghana, Kenya and Nepal." YouthSave Research Report. St. Louis: Washington University. csd.wustl.edu/ Publications/Documents/RR15-01.pdf.

Kagitcibasi, Cigdem, Diane Sunar et Sevda Bekman. 2001. "Long-term effects of early intervention: Turkish low-income mothers and children," Journal of Applied Developmental Psychology 22(4): 333-361.

Keino, Susan, Guy Plasqui, Grace Ettyang et Bart van den Borne. 2014. "Determinants of stunting and overweight among young children and adolescents in sub-Saharan Africa," Food \& Nutrition Bulletin 35(2): 167-178.

Kessler, Ronald C., G. Paul Amminger, Sergio Aguilar-Gaxiola, Jordi Alonso, Sing Lee et T. Bedirhan Ustün. 2007. "Age of onset of mental disorders: A review of recent literature," Current Opinion in Psychiatry 20(4): 359-364. doi:10.1097/ YCO.Ob013e32816ebc8c. 
Kieling, Christian, Helen Baker-Henningham, Myron Belfer, et al. 2011. "Child and adolescent mental health worldwide: Evidence for action," The Lancet 378(9801): 1515-1525.

Knerr, Wendy, Frances Gardner et Lucie Cluver. 2013. "Improving positive parenting skills and reducing harsh and abusive parenting in low-and middle-income countries: A systematic review," Prevention Science 14(4): 352-363.

Kohl, R. 2011. "An analytical framework for scaling up pilots in public health," in Richard A. Cash, A. Mushtaque, R. Chowdhury, George B. Smith, Faruque Ahmed (eds.), From One to Many: Scaling up Health Programs in Low Income Countries, pp. 241-254. Dhaka, Bangladesh: University Press Limited.

Konstantopoulos, Wendy Macias, Roy Ahn, et al. 2013. "An international comparative public health analysis of sex trafficking of women and girls in eight cities: Achieving a more effective health sector response," Journal of Urban Health 90(6): 1194-1204.

Kremer, Michael et Alaka Holla. 2009. “Improving education in the developing world: What have we learned from randomized evaluations?" Annual Review of Economics 1: 513.

Kumpfer, Karol L., Methinin Pinyuchon, Ana Teixeira de Melo et Henry O. Whiteside. 2008. "Cultural adaptation process for international dissemination of the strengthening families program," Evaluation \& the Health Professions 31(2): 226-239.

Lardoux, Solène et Afiwa N'Bouke. 2013. “Reaching adolescents and youth in Burkina Faso, Guinea-Bissau and Mauritania," African Journal of Reproductive Health 17(1): 73-84.

Larkin, Heather, Brooke A. Beckos et Joseph J. Shields. 2012. "Mobilizing resilience and recovery in response to Adverse Childhood Experiences (ACE): A Restorative Integral Support (RIS) case study," Journal of Prevention \& Intervention in the Community 40(4): 335-346. doi:10.1080/10852 352.2012.707466.

Lau, Patrick W.C., Erica Y. Lau, Del P. Wong et Lynda Ransdell. 2011. "A systematic review of information and communication technology-based interventions for promoting physical activity behavior change in children and adolescents," Journal of Medical Internet Research 13(3): e48. doi:10.2196/jmir.1533.

Leach, F., M. Dunne et F. Salvi. 2014. School-Related Gender-Based Violence: A Global Review of Current Issues and Approaches in Policy, Programming and Implementation Responses to School-Related Gender-Based Violence (SRGBV) for the Education Sector. Paris: UNESCO.
Leen, Eline, Emma Sorbring, Matt Mawer, Emma Holdsworth, Bo Helsing et Erica Bowen. 2013. "Prevalence, dynamic risk factors and the efficacy of primary interventions for adolescent dating violence: An international review," Aggression and Violent Behavior 18(1): 159-174.

Logan, T.K., Robert Walker et Gretchen Hunt. 2009. "Understanding human trafficking in the United States," Trauma, Violence, \& Abuse 10(1): 3-30.

Lundgren, Rebecka, Miranda Beckman, Surendra Prasad Chaurasiya, Bhawna Subhedi et Brad Kerner. 2013. "Whose turn to do the dishes? Transforming gender attitudes and behaviours among very young adolescents in Nepal," Gender \& Development 21(1): 127-145.

Malhotra, Anju, Ann Warner, Allison McGonagle et Susan Lee-Rife. 2011. "Solutions to end child marriage." Washington, DC: International Center for Research on Women. www.icrw.org/files/publications/Solutions-to-End-Child-Marriage.pdf.

Mandell, L. 2009. “Starting younger: Evidence supporting the effectiveness of personal financial education for pre-high school students." Seattle: University of Washington and the Aspen Institute. www.nationaltheatre.com/ntccom/pdfs/ financialliteracy.pdf.

Marie Stopes International. Ethics Review Committee (ERC) documents. https://mariestopes.org/ data-research/ethics-review-committee.

Marston, Cicely et Eleanor King. 2006. "Factors that shape young people's sexual behaviour: A systematic review," The Lancet 368(9547): 1581-1586.

Martin, Megan. 2012. "The Arab Spring: Youth, freedom and the tools of technology." Al Arabiya News. April 6. english.alarabiya.net/ views/2012/04/06/205837.html.

McClain, Natalie M. et Stacy E. Garrity. 2011. "Sex trafficking and the exploitation of adolescents," Journal of Obstetric, Gynecologic, \& Neonatal Nursing 40(2): 243-252.

Mejia, Anilena, Rachel Calam et Matthew R. Sanders. 2012. "A review of parenting programs in developing countries; Opportunities and Challenges for preventing emotional and behavioral difficulties in children," Clinical Child and Family Psychology Review 15(2): 163-175.

.2014. "Examining delivery preferences and cultural relevance of an evidence-based parenting program in a low-resource setting of Central America: Approaching parents as consumers," Journal of Child and Family Studies 24(4): 1004-1015. doi:10.1007/s10826-014-9911-x.

Mensch, Barbara, Susheela Singh et John B. Casterline. 2005. "Trends in the timing of first marriage among men and women in the developing world." New York: Population Council. 
Mensch, Barbara, Erica Soler-Hampejsek, Christine A. Kelly et Paul C. Hewett. 2011. "Transitions to adulthood in rural Malawi: Estimating the sequencing of sexual initiation, school leaving and marriage." Paper presented at the 2011 Meeting of the Population Association of America, Washington, DC.

Mensch, Barbara, Erica Soler-Hampejsek, Christine A. Kelly, Paul C. Hewett et Monica J. Grant. 2014. "Challenges in measuring the sequencing of life events among adolescents in Malawi: A cautionary note," Demography 51(1): 277-285. doi:10.1007/s13524-013-0269-2.

Meyer-Rochow, Victor Benno. 2009. "Food taboos: Their origins and purposes," Journal of Ethnobiology and Ethnomedicine 5:18. doi:10.1186/17464269-5-18.

Mikton, Christopher et Alexander Butchart. 2009. "Child maltreatment prevention: A systematic review of reviews," Bulletin of the World Health Organization 87(5): 353-361. doi:10.1590/ S0042-96862009000500012.

Mmari, Kristin et Nan Astone. 2014. "Urban adolescent sexual and reproductive health in low-income and middle-income countries," Archives of Disease in Childhood 99(8) 778-782.

Mmari, Kristin, Robert Blum, Freya Sonenstein, Beth Marshall, Heena Brahmbhatt, Emily Venables, Sinead Delany-Moretlwe, et al. 2014. "Adolescents' perceptions of health from disadvantaged urban communities: Findings from the WAVE study," Social Science \& Medicine 104: 124-132.

More, Sarika, V. B. Shivkumar, Nitin Gangane et Sumeet Shende. 2013. "Effects of iron deficiency on cognitive function in school going adolescent females in rural area of Central India," Anemia. doi:10.1155/2013/819136.

Morris, Jodi, Myron Belfer, Amy Daniels, et al. 2011. "Treated prevalence of and mental health services received by children and adolescents in 42 lowand-middle-income countries," Journal of Child Psychology and Psychiatry 52(12): 1239-1246.

Mudaly, N. et C. Goddard. 2009. “Ethics involving children who have been abused in child abuse research," International Journal of Children's Rights 17(2): 261-281.

Mukuddem-Petersen, J. et H. Salome Kruger. 2004. "Association between stunting and overweight among 10-15-y-old children in the North West Province of South Africa: The THUSA BANA Study." International Journal of Obesity and Related Metabolic Disorders 28(7): 842-851. doi:10.1038/sj.ijo.0802586.

National Children's Advocacy Center (NCAC). 2013. "As a matter of fact: The relationship between child maltreatment and academic achievement." Huntsville: NCAC.
Nguyen, Trang. 2008. "Information, role models and perceived returns to education: experimental evidence from Madagascar." Unpublished.

O'Prey, L. et D. Shepard. 2014. "Financial education for children and youth: A systematic review and meta-analysis." Aflatoun Working Paper, 2014.lc.

Organisation mondiale de la Santé (OMS). 2001. "Prevention of child abuse and neglect: Making the links between human rights and public health." Genève: OMS.

- 2009. "Preventing violence through the development of safe, stable and nurturing relationships between children and their parents and caregivers." Genève: OMS

. 2011. "WHO ethical and safety guidance notes for research with adolescents: Report of a WHO technical consultation." Genève: OMS. whqlibdoc.who.int/hq/2011/who_rhr_11.11_eng. pdf.

- 2012a. "Adolescent mental health: Mapping actions of nongovernmental organizations and other international development organizations." Genève: OMS. whqlibdoc.who.int/publications/ 2012/9789241503648_eng.pdf.

. 2012b. "Global health estimates." Genève: OMS. www.who.int/healthinfo/global_burden_ disease/estimates/en/.

- 2013. "Health data and statistics." Genève: OMS. www.who.int/healthinfo/statistics/en/. . 2014a. "Global health estimates 2013 summary tables: DALYS, YLLs and YLDs by cause, age and sex by WHO regional group and World Bank income classification, 2000-2012 (provisional estimates)." Genève: OMS.

. 2014b. "Health for the World's Adolescents: A Second Chance in the Second Decade." Genève: OMS. apps.who.int/adolescent/second-decade.

- 2015. "Interim Report of the Commission on Ending Childhood Obesity." Genève: OMS.

WHO, World Psychiatric Association (WPA), et International Association for Child et Adolescent Psychiatry and Allied Professions (IACAPAP). 2005. Child and Adolescent Mental Health Resources: Global Concerns-Implications for the Future. Genève: OMS. www.who.int/ mental_health/resources/Child_ado_atlas.pdf.

Pasick, Rena J., Nancy J. Burke, Judith C. Barker, et al. 2009. "Behavioral theory in a diverse society: Like a compass on Mars," Health Education \& Behavior 36(5 Suppl.): 11S-35S.

Patel, Vikram, Niall Boyce, Pamela Y. Collins, Shekhar Saxena et Richard Horton. 2011. "A renewed agenda for global mental health," The Lancet 378(9801): 1441-1442. doi: 10.1016/S01406736(11)61385-8. 
Patel, Vikram, Alan J. Flisher, Sarah Hetrick et Patrick McGorry. 2007. "Mental health of young people: A global public-health challenge," The Lancet 369(9569): 1302-1313. doi:10.1016/S01406736(07)60368-7.

Patel, Vikram, Helen A. Weiss, Neerja Chowdhary, et al. 2011. "Lay health worker led intervention for depressive and anxiety disorders in India: Impact on clinical and disability outcomes over 12 months," The British Journal of Psychiatry 199(6): 459-466.

Patton, George C., Carolyn Coffey, Claudia Cappa, et al. 2012. "Health of the world's adolescents: A synthesis of internationally comparable data," The Lancet 379(9826): 1665-1675. doi:10.1016/ S0140-6736(12)60203-7.

Peacock, Dean et Gary Barker. 2014. "Working with men and boys to prevent gender-based violence principles, lessons learned, and ways forward," Men and Masculinities 17(5): 578-599.

Peña, R., M. Quintanilla, K. Navarro, J. Martínez, V. Castillo, W. Pérez et C. Källestäl. 2008. “Evaluating a peer intervention strategy for the promotion of sexual health-related knowledge and skills in 10- to 14-year-old girls. Findings from the 'Entre Amigas' project in Nicaragua," American Journal of Health Promotion 22(4): 275-281.

Pereda, Noemi, Georgina Guilera, Maria Forns et Juana Gómez-Benito. 2009a. “The international epidemiology of child sexual abuse: A continuation of Finkelhor (1994)," Child Abuse \& Neglect 33(6): 331-342.

. 2009b. "The prevalence of child sexual abuse in community and student samples: A meta-analysis," Clinical Psychology Review 29(4): 328-338.

Pereznieto, P., C. Harper, B. Clench et J. Coarasa. 2010. "The economic impact of school violence: A report for Plan International." London: Plan International and Overseas Development Institute. www.ungei.org/files/violence.pdf.

Phetla, Godfrey, Joanna Busza, James R. Hargreaves, et al. 2008. "'They have opened our mouths': Increasing women's skills and motivation for sexual communication with young people in rural South Africa," AIDS Education and Prevention, 6(December): 504-518. doi:10.1521/ aeap.2008.20.6.504.

Phillips, A.E., G.B. Gomez, M.C. Boily et G.P. Garnett. 2010. "A systematic review and metaanalysis of quantitative interviewing tools to investigate self-reported HIV and STI associated behaviors in low- and middle- income countries," International Journal of Epidemiology 39(6): 1541-1555.

Piperata, Barbara A., Kammi K. Schmeer, Craig Hadley et Genevieve Ritchie-Ewing. 2013. “Dietary inequalities of mother-child pairs in the rural Amazon: Evidence of maternal-child buffer- ing?" Social Science \& Medicine 96: 183-191. www.sciencedirect.com/science/article/pii/ S0277953613004231.

Population Council. 2015. “Adolescent Girls' Programming Toolkits." New York: Population Council. www.popcouncil.org/research/ adolescent-girls-programming-toolkits.

-2000-15. "The adolescent experience indepth: Using data to identify and reach the most vulnerable young people." New York: Population Council. Accessed June 16. www.popcouncil.org/ research/the-adolescent-experience-in-depthusing-data-to-identify-and-reach-th.

Population Reference Bureau (PRB). 2015. “Addressing risk factors for noncommunicable diseases among young people in Africa: Key to prevention and sustainable development." Washington, DC: Population Reference Bureau.

Psaki, Stephanie, Barbara Mensch et Erica SolerHampejsek. 2015. "The effects of violence in school and at home on education outcomes in rural Malawi: A longitudinal analysis." Paper presented at the 2015 Annual Meeting of the Population Association of America. San Diego, CA.

Puska, Pekka. 2004. "The global strategy on diet, physical activity \& health: 10 years on." Cancer Prevention Research and Policy Blog. wcrf.org/int/blog/articles/2014/05/globalstrategy-diet-physical-activity-health-10-years.

Raftree, Linda et K. Bachan. 2013. “Integrating information and communication technologies into communication for development strategies to support and empower marginalized adolescent girls." New York: UNICEF.

Robles, Omar. 2014. "I'm here: Adolescent girls in emergencies report." New York: Women's Refugee Commission. womensrefugeecommission. org/resources/document/1078-i-m-here-reportfinal-pdf.

Santhya, K. G. et Shireen J. Jejeebhoy. 2015. “Sexual and reproductive health and rights of adolescent girls: Evidence from low- and middle-income countries," Global Public Health 10(2): 189-221. doi:10.1080/17441692.2014.986169.

Save the Children. 2008. "Kit of tools for participatory research and evaluation with children, young people and adults." Oslo, Norway: Save the Children.

Save the Children Denmark, Ethiopian Ministry of Education et Ethiopian Ministry of Women's Affairs. 2008. "A study on violence against girls in primary schools and its impacts on girls' education in Ethiopia." Addis Ababa: Save the Children.

Sawyer, Susan M., Rima A. Afifi, Linda H. Bearinger, Sarah-Jayne Blakemore, Bruce Dick, Alex C. Ezeh et George C. Patton. 2012. "Adolescence: A foundation for future health," The Lancet 379(9826): 1630-40. doi:10.1016/S0140-6736(12)60072-5. 
Schauer, Elizabeth et Thomas Elbert. 2010. "Psychological impact of child soldiering," in Erin Martz (ed.), Trauma Rehabilitation After War and Conflict, pp. 311-360. New York: Springer Science + Business Media.

Schenk, K. et J. Williamson. 2005. "Ethical approaches to gathering information from children and adolescents in international settings: Guidelines and resources." Washington, DC: Population Council.

Schwartz, David, Jennifer E. Lansford, Kenneth A. Dodge, Gregory S. Pettit et John E. Bates. 2013. "The link between harsh home environments and negative academic trajectories is exacerbated by victimization in the elementary school peer group," Developmental Psychology 49(2): 305.

Sedlak, A. et al. 2010. "Fourth National Incidence Study of Child Abuse and Neglect (NIS-4) Report to Congress." Washington, DC: US Department of Health and Human Services, Administration for Children and Families.

Selim, Mona, Nahla G. Abdel-Tawab, Khaled El Sayed, Asmaa Elbadawy et Heba El Kalaawy. 2013. "The Ishraq Program for out-of-school girls: From pilot to scale-up." Cairo, Egypt: Population Council.

Shared Hope International. 2009. The National Report on Domestic Minor Sex Trafficking: America's Prostituted Children. Vancouver: Shared Hope International.

Shumba, Almon. 2002. "The nature, extent and effects of emotional abuse on primary school pupils by teachers in Zimbabwe," Child Abuse \& Neglect 26(8): 783-791.

Simmons, R., P. Faijans et L. Ghiron. 2007. “Scaling up health service delivery: From pilot innovations to policies and programmes." Geneva: World Health Organization.

Slopen, Natalie, Garrett Fitzmaurice, David R. Williams et Stephen E. Gilman. 2010. "Poverty, food insecurity, and the behavior for childhood internalizing and externalizing disorders," Journal of the American Academy of Child \& Adolescent Psychiatry 49(5): 444-452.

Small, Kevonne et Justice Policy Center. 2008. An Analysis of Federally Prosecuted CSEC Cases Since the Passage of the Victims of Trafficking and Violence Protection Act of 2000: Final Report. Washington, DC: Urban Institute, Justice Policy Center.

Sommer, Marni. 2011. “An early window of opportunity for promoting girls' health: Policy implications of the girl's puberty book project in Tanzania," International Electronic Journal of Health Education 14: 77-92.

Staurowsky, E., M.J. DeSousa, G. Ducher, N. Gentner, K. Miller, S. Shakib, N. Theberge et N. Williams. 2015. "Her life depends on it III: Sport, physical activity, and the health and well-being of Amer- ican girls and women." East Meadow, NY: Women's Sports Foundation. www.womenssportsfoundation.org/en/sitecore/content/home/ research/articles-and-reports/mental-andphysical-health/her-life-depends-on-it-iii.aspx.

Steinberg, Lawrence. 2014. “The science of adolescent brain development and its implication for adolescent rights and responsibilities," in Jacqueline Bhabha, Human Rights and Adolescence. Pennsylvania Studies in Human Rights. Philadelphia: University of Pennsylvania Press.

Stoltenborgh, Marije, Marinus H. van IJzendoorn, Eveline M. Euser et Marian J. Bakermans-Kranenburg. 2011. "A global perspective on child sexual abuse: Meta-analysis of prevalence around the world," Child Maltreatment 16(2): 79-101.

Sumpter, Colin et Belen Torondel. 2013. "A systematic review of the health and social effects of menstrual hygiene management," PLoS ONE 8(4). doi:10.1371/journal.pone.0062004.

Temin, Miriam et Ruth Levine. 2009. "Start with a girl: A new agenda for global health." Girls Count Series. Washington, DC: Center for Global Development. www.cgdev.org/files/1422899_file_ Start_with_a_Girl_FINAL.pdf.

Temin, Miriam, Mark R. Montgomery, Sarah Engebretsen et Kathryn M. Barker. 2013. "Girls on the move: Adolescent girls \& migration in the developing world. A Girls Count report on adolescent girls." New York: Population Council. www.popcouncil.org/uploads/pdfs/2013PGY_ GirlsOnTheMove.pdf.

Theoklitou, D., N. Kabitsis et A. Kabitsi. 2012. "Physical and emotional abuse of primary school children by teachers," Child Abuse \& Neglect 36(1): 64-70.

UNESCO. 2009. "International technical guidance on sexuality education: An evidence-informed approach for schools, teachers, and health educators." Paris: UNESCO.

2015. "Education for all 2000-2015: Achievements and challenges." Education for All Global Monitoring Report. Paris: UNESCO.

UNESCO Institute for Statistics. 2011. “Early adolescent girls: A global view of participation in lower secondary education." Fact Sheet 11. Paris: UNESCO. www.uis.unesco.org/FactSheets/ Documents/fs11-2011-en.pdf.

- 2015. "A growing number of children and adolescents are out of school as aid fails to meet the mark." UNESCO Paper 22/Fact Sheet 31. Paris: UNESCO. www.uis.unesco.org/Education/ Documents/fs-31-out-of-school-children-en.pdf.

UNFPA. 2010. "Comprehensive sexuality education: Advancing human rights, gender equality and improved sexual and reproductive health." New York: UNFPA. 
UNICEF. 2005. "Early marriage: A harmful traditional practice." New York: UNICEF.

- 2006. "Guidelines on the protection of child victims of trafficking." New York: UNICEF. www.unicef.org/ceecis/0610-Unicef_Victims_ Guidelines_en.pdf.

2007. "A national study on violence against children and young women in Swaziland." Mbabane: UNICEF Swaziland.

2011. "Adolescence: An age of opportunitythe state of the world's children." New York: UNICEF. www.unicef.org/adolescence/files/ SOWC_2011_Main_Report_EN_02092011.pdf.

- 2012a. "Child social and financial education. A Companion to the Child Friendly Schools Manual." New York: UNICEF.

- 2012b. "Global evaluation of life skills education programmes." New York: UNICEF.

2012c. "Progress for children: A report card on adolescents." New York: UNICEF.

2013. "Towards an AIDS-free generation: Children and AIDS Sixth Stocktaking Report." New York: UNICEF.

2014a. "Ending child marriage: Progress and prospects." New York: UNICEF. www.unicef. org/media/files/Child_Marriage_Report_7_ 17_LR.pdf.

- 2014b. "Female genital mutilation/cutting: What might the future hold?" New York: UNICEF.

2014c. "Hidden in plain sight: A statistical analysis of violence against children." New York: UNICEF. www.unicef.org/publications/ index_74865.html.

UNICEF et Collaborating Centre for Operational Research and Evaluation. 2013. National Baseline Survey on Life Experiences of Adolescents. Harare: UNICEF and Zimbabwe National Statistics Agency.

UNICEF, National Center for Injury Prevention and Control, US Centers for Disease Control and Prevention et Kenya National Bureau of Statistics. 2012. Violence Against Children in Kenya: Findings from a 2010 National Survey. Nairobi: UNICEF.

UNICEF, US Centers for Disease Control and Prevention Children's Fund et Université de Muhimbili Health and Allied Sciences. 2011. Violence against Children in Tanzania: Findings from a National Survey 2009. Dar es Salaam: UNICEF Tanzania.

UNODC. 2009. "Guide to implementing family skills training programmes for drug abuse prevention." Vienna: UNODC.

United Nations Department of Economic and Social Affairs-Population Division (UNDESA). 2013. World Population Prospects, the 2012 Revision. esa.un.org/wpp/.
Van Wyk, J.D., M.E. Eloff et P.M. Heyns. 1983. “The evaluation of an integrated parent-training program," The Journal of Social Psychology 121(2): 273-281.

Verstraeten, Roosmarijn, Dominique Roberfroid, Carl Lachat, et al. 2012. "Effectiveness of preventive school-based obesity interventions in low- and middle-income countries: A systematic review," The American Journal of Clinical Nutrition. August. doi:10.3945/ajcn.112.035378.

Villarruel, Antonia M., Carol Loveland Cherry, Esther Gallegos Cabriales, David L. Ronis et Yan Zhou. 2008. "A parent-adolescent intervention to increase sexual risk communication: Results of a randomized controlled trial," AIDS Education and Prevention 20(5): 371-383. doi:10.1521/ aeap.2008.20.5.371.

War Child. 2015. "Child soldiers." www.warchild.org. uk/issues/child-soldiers.

Wight, Daniel et Deirdre Fullerton. 2013. “A review of interventions with parents to promote the sexual health of their children," Journal of Adolescent Health 52(1): 4-27.

Williamson, Celia et Michael Prior. 2009. “Domestic minor sex trafficking: A network of underground players in the Midwest," Journal of Child \& Adolescent Trauma 2(1): 46-61.

Women's Refugee Commission (WRC), Johns Hopkins University, Adolescent Reproductive Health Network, International Medical Corps, Save the Children, American University in Beirut. 2014.

"Very young adolescents in humanitarian settings: Examining the sexual and reproductive health needs and risks of girls and boys aged 10-14 in Ethiopia, Lebanon and Thailand." New York: WRC.

Yaqub, Shahin. 2009. “Independent child migrants in developing countries: Unexplored links in migration and development." Florence: UNICEF Innocenti Research Centre. ideas.repec.org/p/ ucf/inwopa/inwopa09-62.html.

Yoder, P. Stanley, Shanxiao Wang et Elise Johansen. 2013. "Estimates of female genital mutilation/ cutting in 27 African countries and Yemen," Studies in Family Planning 44(2): 189-204.

Zolotor, A.J., D.K. Runya, M.P. Dunne, D. Jain, H.R. Petrus, C. Ramirez, E. Volkova, et al. 2009. "ISPCAN Child Abuse Screening Tool Children's Version (ICAST-C): Instrument development and multinational pilot testing," Child Abuse \& Neglect 33(11): 833-841. 



\section{POPULATION}

COUNCIL

Ideés. Preuve. Impact.

One Dag Hammarskjold Plaza

New York, NY 10017

popcouncil.org 